Key Words:

Performance Assessment

Probabilistic

Retention:

Permanent

\title{
PROBABILISTIC SENSITIVITY AND UNCERTAINTY ANALYSIS WORKSHOP SUMMARY REPORT
}

\author{
Roger Seitz, SRNL \\ Bruce Crowe, Battelle Memorial Institute \\ Mike Sully, New Mexico Office of the State Engineer \\ (formerly with Neptune and Co.) \\ Marc Wood, Fluor Hanford
}

JUNE 2008

Savannah River National Laboratory

Washington Savannah River Company

Savannah River Site

Aiken, SC 29808

Prepared for the U.S. Department of Energy Under

Contract Number DE-AC09-96SR18500 


\section{DISCLAIMER}

This report was prepared for the United States Department of Energy under Contract No. DE-AC09-96SR18500 and is an account of work performed under that contract. Neither the United States Department of Energy, nor WSRC, nor any of their employees makes any warranty, expressed or implied, or assumes any legal liability or responsibility for accuracy, completeness, or usefulness, of any information, apparatus, or product or process disclosed herein or represents that its use will not infringe privately owned rights. Reference herein to any specific commercial product, process, or service by trade name, trademark, name, manufacturer or otherwise does not necessarily constitute or imply endorsement, recommendation, or favoring of same by Washington Savannah River Company or by the United States Government or any agency thereof. The views and opinions of the authors expressed herein do not necessarily state or reflect those of the United States Government or any agency thereof.

Printed in the United States of America

Prepared For

U.S. Department of Energy 
Key Words:

Performance Assessment

Probabilistic

Retention:

Permanent

\title{
PROBABILISTIC SENSITIVITY AND UNCERTAINTY ANALYSIS WORKSHOP SUMMARY REPORT
}

\author{
Roger Seitz, SRNL \\ Bruce Crowe, Battelle Memorial Institute \\ Mike Sully, New Mexico Office of the State Engineer \\ (formerly with Neptune and Co.) \\ Marc Wood, Fluor Hanford
}

JUNE 2008

Savannah River National Laboratory

Washington Savannah River Company

Savannah River Site

Aiken, SC 29808 


\begin{abstract}
Stochastic or probabilistic modeling approaches are being applied more frequently in the United States and globally to quantify uncertainty and enhance understanding of model response in performance assessments for disposal of radioactive waste. This increased use has resulted in global interest in sharing results of research and applied studies that have been completed to date. This technical report reflects the results of a workshop that was held to share results of research and applied work related to performance assessments conducted at United States Department of Energy sites. Key findings of this research and applied work are discussed and recommendations for future activities are provided.
\end{abstract}

\title{
ACKNOWLEDGEMENTS
}

The authors would like to acknowledge the programmatic support from Karen Guevara and Martin Letourneau, EM-11 at DOE-HQ. Rosalind Blocker of SRNL deserves a lot of credit for the success of the workshop as a result of her efforts on the approvals, logistics, and actual implementation of the workshop. Howard Pope and Mike Simmons at DOE-SR also provided critical support and assistance with arrangements and approvals to implement this workshop.

The authors would also like to thank several people that have provided review comments on drafts of this report, including Howard Pope of DOE-SR, Phil Meyer of PNNL, Elmer Wilhite, SRNL, and David Esh of the US NRC. While they provided valuable suggestions that improved the quality and clarity of the report, it is emphasized that final responsibility for the content of the report rests with the authors. 


\section{TABLE OF CONTENTS}

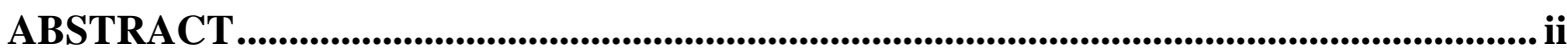

ACKNOWLEDGEMENTS ................................................................................................. ii

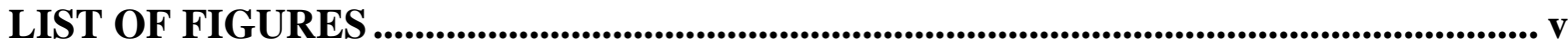

LIST OF TABLES

LIST OF ACRONYMS .............................................................................................................. vi

1.0 EXECUTIVE SUMMARY ........................................................................................ 1

1.1 BACKGROUND $\ldots \ldots \ldots \ldots \ldots \ldots \ldots \ldots \ldots \ldots \ldots \ldots \ldots \ldots \ldots \ldots \ldots \ldots \ldots \ldots \ldots \ldots \ldots \ldots \ldots \ldots \ldots \ldots \ldots \ldots \ldots \ldots \ldots \ldots \ldots \ldots \ldots \ldots 1$

1.2 WORKSHOP OBJECTIVES ............................................................................... 1

1.3 WORKSHOP APPROACH..................................................................................... 2

1.4 KEY POINTS FROM PRESENTATIONS AND DISCUSSIONS ......................... 3

1.4.1 Introductory Remarks ........................................................................................ 4

1.4.2 Session 1 - Development of Distributions .................................................. 5

1.4.3 Session 2 - Model Abstraction ......................................................................6 6

1.4.4 Session 3 - Implementation of Sensitivity and Uncertainty Analysis................. 6

1.4.5 Session 4-Policy Implications ........................................................................ 7

1.5 WORKSHOP CONCLUSIONS AND RECOMMENDATIONS ............................ 7

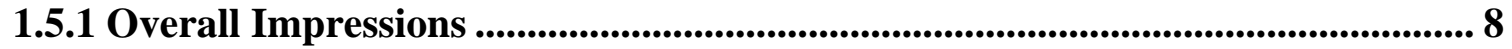

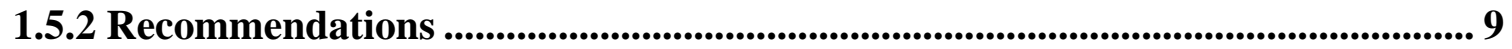

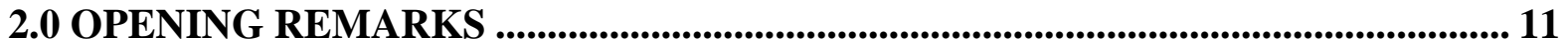

2.1 INTRODUCTORY PRESENTATIONS ................................................................. 11

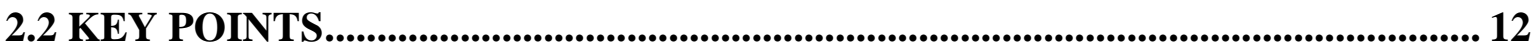

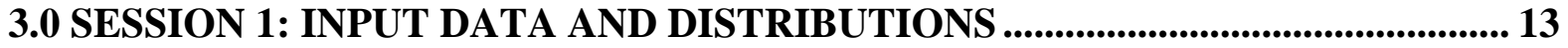

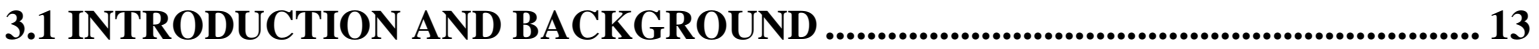

3.2 PRESENTATIONS ................................................................................................... 14

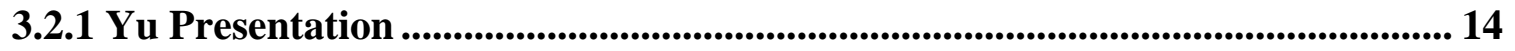

3.2.2 Esh Presentation.................................................................................................... 15

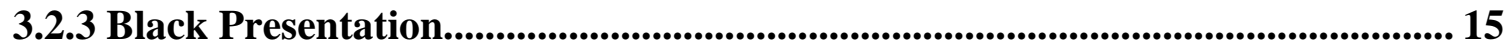

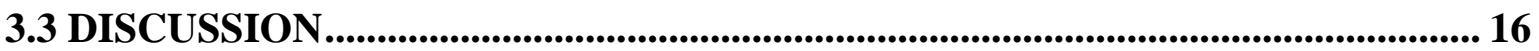

3.4 RECOMMENDATIONS ................................................................................... 17

4.0 SESSION 2 MODEL ABSTRACTION ............................................................ 19

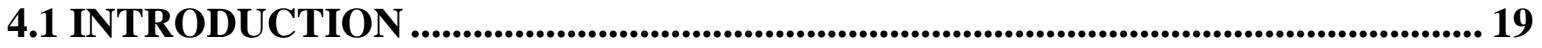

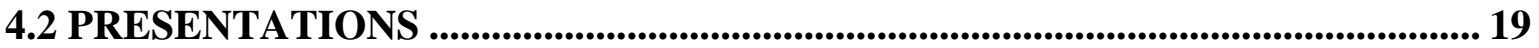

4.2.1 Flach and Taylor Presentation......................................................................... 20

4.2.2 Stauffer Presentation ............................................................................................. 20

4.2.3 Kozak Presentation .................................................................................... 21

4.3 DISCUSSION.................................................................................................................. 21

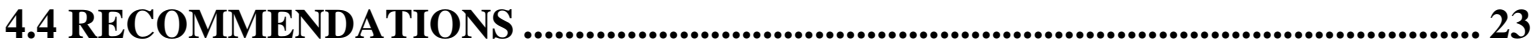

5.0 SESSION 3: IMPLEMENTATION OF SENSITIVITY AND UNCERTAINTY

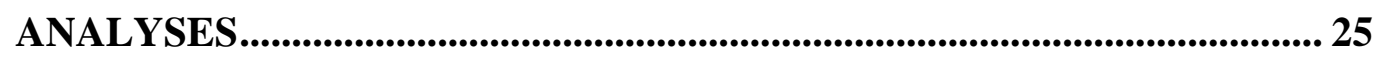

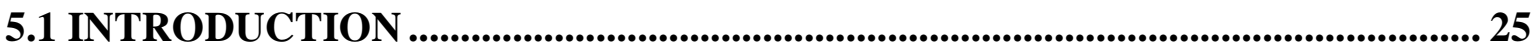

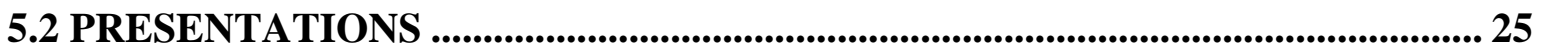

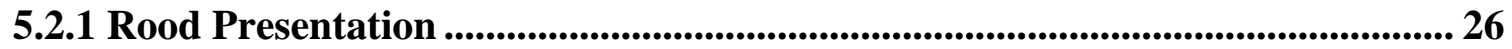

5.2.2 Black Presentation...................................................................................................... 27

5.2.3 Tauxe Presentation................................................................................. 28 


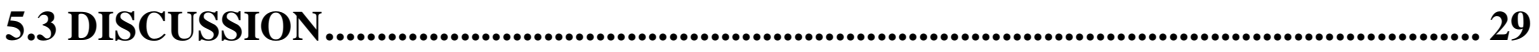

5.4 RECOMMENDATIONS ............................................................................................. 31

6.0 SESSION 4: POLICY IMPLICATIONS................................................................. 33

6.1 INTRODUCTION ................................................................................................................. 33

6.2 PRESENTATIONS ..................................................................................................... 33

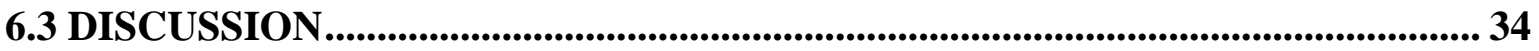

6.4 RECOMMENDATIONS ............................................................................................. 34

7.0 CONCLUSIONS AND RECOMMENDATIONS.......................................................... 36

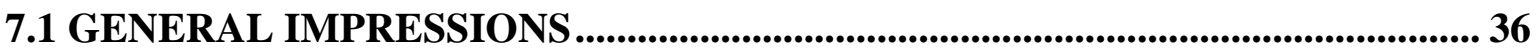

7.2 OVERALL RECOMMENDATIONS............................................................................ 37

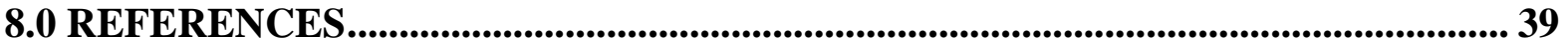

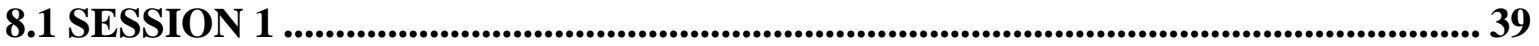

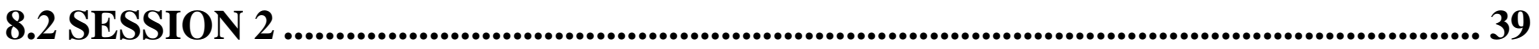

APPENDIX A. WORKSHOP AGENDA.............................................................. 40

APPENDIX B. LIST OF PARTICIPANTS ….................................................... 43

APPENDIX C. PRESENTATIONS ..................................................................................... 47

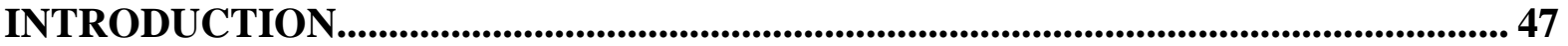

SESSION 1 - INPUT DATA AND DISTRIBUTIONS ........................................................... 53

SESSION 2 - MODEL ABSTRACTION ............................................................................. 73

SESSION 3 - IMPLEMENTATION OF PROBABILISTIC SENSITIVITY AND

UNCERTAINTY ANALYSIS ............................................................................... 91 


\section{LIST OF FIGURES}

Figure 1. Bruce Crowe, NTS, leading the panel discussion after Session 1 (Paul Black, Charley $\mathrm{Yu}$, and David Esh are the Panel Members from left to right.)........................... 2

Figure 2. Martin Letourneau, EM-11 - Opening Remarks ................................................. 11

\section{LIST OF TABLES}

Table 1. Summary of Rapporteurs and Speakers ............................................................. 3 


\section{LIST OF ACRONYMS}

$\begin{array}{ll}\text { ACRI } & \text { Analytic \& Computational Research, Inc. } \\ \text { CBPT } & \text { Convolution based particle tracking } \\ \text { DNFSB } & \text { Defense Nuclear Facility Safety Board } \\ \text { DOE-HQ } & \text { Department of Energy - Headquarters } \\ \text { DOE-HS } & \text { Department of Energy - Homeland Security } \\ \text { DOE-SR } & \text { Department of Energy - Savannah River } \\ \text { EPA } & \text { Environmental Protection Agency } \\ \text { ISCORS } & \text { Interagency Steering Committee on Radiation Standards } \\ \text { LANL } & \text { Los Alamos National Laboratory } \\ \text { LFRG } & \text { Low-Level Waste Disposal Facility Federal Review Group } \\ \text { LLW } & \text { Low Level Waste } \\ \text { NDDA } & \text { Ronald W. Reagan National Defense Authorization Act } \\ \text { PA } & \text { Performance Assessment } \\ \text { PDF } & \text { Probability Density Function } \\ \text { NRC } & \text { Nuclear Regulatory Commission } \\ \text { NTS } & \text { Nevada Test Site } \\ \text { OFAT } & \text { One factor at a time } \\ \text { PRA } & \text { Probabilistic Risk Assessment }\end{array}$


WSRC-STI-2008-00333, REVISION 0

\subsection{EXECUTIVE SUMMARY}

\subsection{BACKGROUND}

Stochastic or probabilistic modeling approaches are being applied more often to quantify uncertainty and enhance understanding of model response in performance assessments (PAs) for low-level waste (LLW) disposal under DOE Order 435.1 and for waste determinations under the Ronald W. Reagan National Defense Authorization Act (NDAA) for Fiscal Year 2005 Section 3116. Three PAs recently reviewed by the Low-Level Waste Disposal Facility Federal Review Group (LFRG) include probabilistic components and there is a strong desire on the part of the United States Nuclear Regulatory Commission (NRC) to use risk-informed approaches for tank closure PAs.

The timing was deemed to be nearly ideal for an opportunity to gather experts from DOE sites, the NRC and various contractors to review and share experiences and lessons learned from existing PAs and to discuss areas where improvements can be made based on these experiences. A workshop also provides the opportunity to promote communication among those conducting PAs to enhance consistency in approaches being used, where it is appropriate, and to establish a network of professionals working in the field.

The workshop was attended by 65 people including DOE Field Staff and staff from EM and HS at DOE HQ, NRC staff, Site Contractor Staff as well as independent consultants providing technical support to DOE Sites. The audience included a mix of people involved in the conduct of assessments and those responsible for oversight of assessments. The audience had substantial experience conducting deterministic and probabilistic assessments.

\subsection{WORKSHOP OBJECTIVES}

Five high-level objectives were identified for the workshop:

- Review applications of probabilistic performance assessments and share technical perspectives, lessons learned and challenges to implementation,

- Provide a forum for practitioners to identify good practices and share experiences,

- Improve consistency in application of probabilistic assessments,

- Address key implementation and policy issues,

- Identify references for information that can help improve the application of probabilistic approaches. 


\subsection{WORKSHOP APPROACH}

The workshop was divided into four topical areas of interest based on lessons learned from LFRG reviews to date:

- Session 1 - Development of distributions for input parameters,

- Session 2 - Abstraction of details from complex groundwater and vadose zone models into simplified models amenable to probabilistic assessments,

- Session 3 - Implementation of sensitivity and uncertainty analysis, and

- Session 4 - Policy issues associated with implementation.

Half-day sessions were dedicated to each topical area. Each session included two or three brief presentations to provide perspective on lessons learned and areas where guidance may be needed. The speakers were selected based on experience with practical involvement in the conduct and review of low-level waste performance assessments. The emphasis of the presentations was on methods for implementing the different topical issues, and to provide an opportunity for some discussion of assessment approaches and probabilistic modeling concepts and tools. A Rapporteur was selected to lead each session and moderate discussions among the participants.

Key points raised during the discussions were recorded and summarized with the group. Lively discussions followed each session and highlighted differing opinions and experiences for the implementation of each topical area (Figure 1). A closing discussion was held to summarize key concepts and recommendations from the workshop.

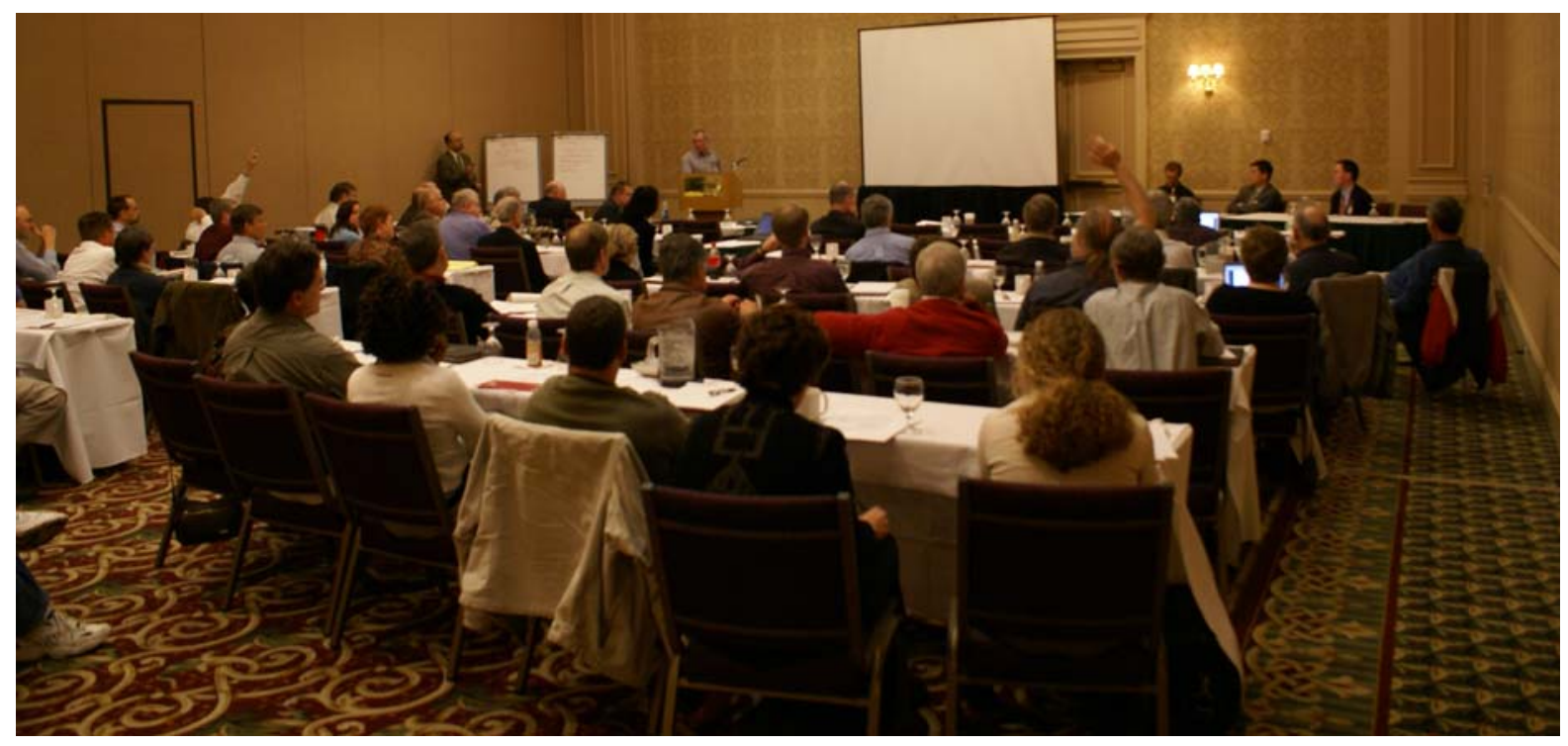

Figure 1. Bruce Crowe, NTS, leading the panel discussion after Session 1 (Paul Black, Charley Yu, and David Esh are the Panel Members from left to right.) 


\subsection{KEY POINTS FROM PRESENTATIONS AND DISCUSSIONS}

This section includes a summary of the content of the workshop and key topics from the presentations and discussions. Table 1 is an overview of the Rapporteurs and speakers for the workshop. Details from presentations and discussions in the individual sessions and concluding remarks are provided in Chapters $2-7$.

Table 1. Summary of Rapporteurs and Speakers

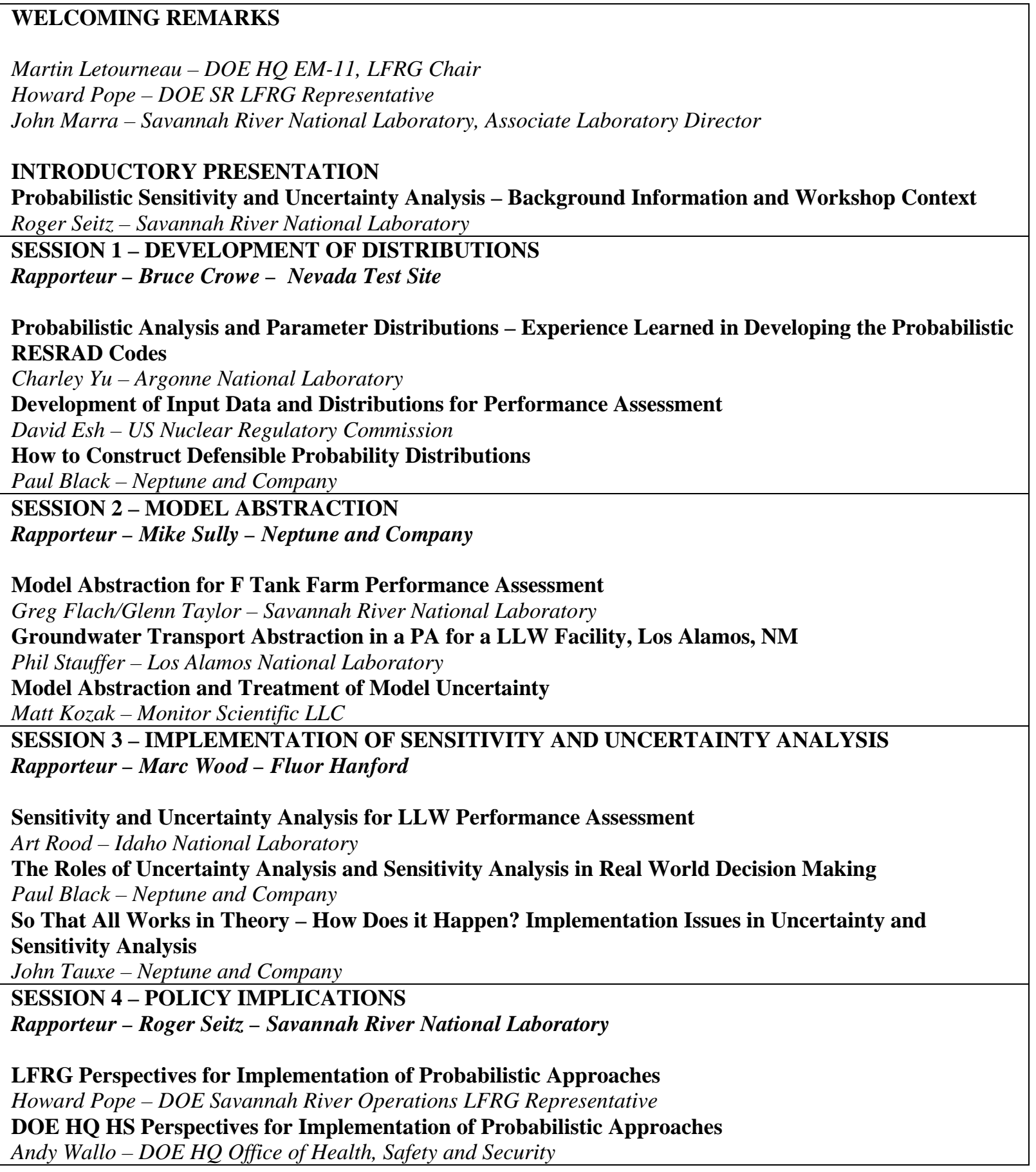




\subsubsection{Introductory Remarks}

\section{Key points from the introductory remarks:}

- Promote improved consistency by bringing the PA community together on a regular basis to share experiences and lessons learned, showcase good modeling practices and encourage communication among practitioners,

- NRC supports increased use of probabilistic approaches for Tank Closure PAs and DOE sites are starting to use probabilistic approaches for LLW disposal facility PAs,

- Multiple performance assessments are underway and improved communication and sharing of experiences will improve consistency in implementation of deterministic and probabilistic approaches,

- Importance of maintaining perspective of performance measures relative to background and other radiation exposure limits,

- Decision based approach for performance assessment rather than a modeling exercise,

- Utility of what is being called a "hybrid" approach for PAs taking advantage of benefits of both deterministic and probabilistic models, and

- Critical role of sensitivity analysis to focus reviewers' attention on key parameters and to identify the most beneficial areas for future work. The term "importance analysis" is used to reflect the way sensitivity analysis is implemented in PAs as a decision-focused application that is a focused and enhanced version of traditional sensitivity analysis. 


\subsubsection{Session 1 - Development of Distributions}

\section{Key points from the presentations and discussions were:}

- Importance of using site-specific information for development of probability density functions for input parameters,

- Discussion of available resources for data and the potential value of conducting retrospective reviews of data and approaches from existing analyses,

- Importance of representativeness of distributions (start simple, represent the uncertainty of data limitations, but recognize that wide distributions can bias the distribution of results),

- Role of temporal and spatial averaging, and parameter correlations in developing appropriate input distributions,

- Using a risk-informed perspective involving a focus on continuing improvement and refinement of key parameters that impact decisions or performance objectives,

- Benefits of a data resource that includes links to the input data and the conceptual basis for developing the distributions used in assessments (e.g., literature references and/or developing a complex-wide resource of data references, potentially a Wikipedia-type approach),

- Discussion of available guidance for development of distributions and associated challenges,

- Debate about consideration of uncertainties in dose factors and probabilities of scenarios,

- Healthy skepticism about the use of probabilistic models and their strengths and weakness compared to traditional use of deterministic models, and

- Need for further detailed discussions and guidance regarding approaches for development of distributions (potential follow-on workshop). 


\subsubsection{Session 2 - Model Abstraction}

Key points from the presentation and discussions were:

- Difficulties in interpreting when a system is amenable to simplification, and establishing a conceptual model for simplified systems,

- Risks of simplification in the context of defensibility of the model - the simiplified model should be shown to adequately represent the more complex deterministic model,

- Recognition of additional benefits of having a simplified model (e.g., facilitate rapid turnaround for special analyses, ease of explanation to stakeholders, quality checks for both the simple and complex model, composite analyses),

- Issue of determining when a deterministic approach is sufficient,

- Benefit of conducting retrospective reviews of PAs to date to collect information on successful simplifications, use of multiple conceptual models, etc.,

- Need for recommendations regarding a structured approach for model abstraction with basic guidelines for acceptability, and

- Value of intercomparisons with other codes and field data to further build confidence in complex and simplified models.

\subsubsection{Session 3 - Implementation of Sensitivity and Uncertainty Analysis}

Key points from this session included:

- The analysis needs to be focused on the objective of the PA (informing a decision regarding compliance),

- Distributions should be consistent with supporting information, recognizing that broad distributions can lead to skewed results. Depending on the information available, it is desirable to focus on average behavior in distributions to avoid unnecessarily extreme skewing of results,

- General agreement that processes exist for sensitivity and uncertainty analysis, but need some customization for unique issues with PAs,

- Importance of using an iterative approach in which sensitivity analyses are used to identify key parameters or assumptions requiring additional data gathering and/or refinement of input parameters in each successive iteration,

- Concept of importance analysis which reflects a sensitivity analysis that is focused on sensitive assumptions relative to conclusions from the modeling, not necessarily global sensitivities in the model,

- Role of a robust sensitivity analysis as part of a demonstration that the system dynamics are relatively well understood, thereby increasing confidence that the uncertainty analysis, and the PA in general, are reliable and sufficient for use in decision-making, 
- Benefits of a "hybrid" approach that uses both deterministic and probabilistic calculations to better inform a decision,

- Difficulties in conducting sensitivity analyses for non-linear systems and specialized probabilistic methods which can be applied to non-linear systems

- Guidelines on how to know when an abstracted model sufficiently represents the system being addressed to be used for a sensitivity analysis,

- Difficulties in addressing low probability scenarios, multiple failure cases, and multiple conceptual models in a deterministic framework, and

- Importance of recognizing that different assumptions will be most sensitive depending on the time in the analysis (e.g., factors affecting mobile radionuclides are more significant at early times) and the need to conduct multiple sensitivity cases for different peaks in the analysis.

\subsubsection{Session 4-Policy Implications}

Key points from the discussion included:

- Difficulties in applying probabilistic results other than the mean and median for compliance assessments against deterministic standards,

- Fundamental questions of how to address cases where results after the time of compliance exceed a standard (applies to both deterministic and probabilistic modeling approaches),

- Need to recognize that the intent of PA is to inform decisions, not force decisions by setting too many specific numerical criteria. Probabilistic modeling results provide increased quantification of uncertainty in model output, which can be useful to provide broader perspective regarding the range of potential results. However, probabilistic distributions of results need to be applied with appropriate cautions, especially when interpreting the significance of tails of distributions.

- Need for guidance on how to address low-probability what-if cases and situations involving multiple failure modes or multiple conceptual models,

- Parallels between safety analysis for both deterministic and probabilistic approaches and comparisons with the "hybrid" approach that has been used more recently for PAs, and

- Need for guidance on conduct of screening and development of waste concentration and inventory limits using probabilistic approaches.

\subsection{WORKSHOP CONCLUSIONS AND RECOMMENDATIONS}

In the opening remarks for the Workshop, it was pointed out that it had been several years since such a gathering of people involved in performance assessments had been held. In the context of improving consistency, the importance of fostering improved communication among the people conducting assessments was recognized by all. 


\subsubsection{Overall Impressions}

The presentations and discussions highlighted the fact that there remains healthy skepticism regarding both probabilistic and deterministic approaches. The group did not see this as a negative. The consensus was this skepticism simply reflects the need for PA practitioners to demonstrate that a thoughtful approach has been used for the assessment rather than relying on a technique to do the thinking. The importance of conducting PAs that are focused on the specific objectives (generally, informing a decision regarding compliance) rather than treating the effort as a modeling exercise was also emphasized many times. PA models can be so complex, it is essential to keep such an objective in mind throughout the process to avoid getting sidetracked on activities that do not contribute to achieving the real goal.

The importance of an iterative approach using sensitivity analysis as a tool was highlighted throughout the discussions and presentations. Although people tend to think of uncertainty analysis first, it was apparent that the sensitivity analysis aspect of the work was a critical consideration for the PA process. The role of sensitivity analysis in identifying future work that has the best potential to benefit the PA and also to guide reviewers to key aspects of the analysis was consistently highlighted. The need for the sensitivity analysis (and the PA in general) to be focused on the decision to be made was highlighted numerous times as well. These considerations have resulted in the term "importance analysis" being used to emphasize that it is not simply global model sensitivity, but identifying sensitive parameters specific to the decision to be made. Approaches for implementation of the sensitivity analysis part of the PA were clearly an area of interest.

A few key technical/policy areas were the subject of much discussion. Concerns about how input distributions are being developed were apparent. Several examples were discussed that illustrated how problems with the definition of distributions have resulted in strange output for the PA models that were resolved through further refinement of the input distributions. There was a lot of discussion about how to know when it is necessary to use a probabilistic approach. There is some expense and time associated with developing a probabilistic model and the inputs needed, so there needs to be a recognized benefit. The general feeling was that it is difficult to set specific criteria, a priori. The consensus seemed to be more along the lines that it is important to recognize the points where the benefits outweigh the costs. Along those lines, it was discussed that there can be many secondary benefits of developing a simplified model amenable to a probabilistic approach, beyond simply enabling the probabilistic calculations. The need to highlight these benefits was identified.

One of the important challenges in adopting a probabilistic approach is developing the model that is amenable to the multiple realizations required for a probabilistic approach. One session was devoted to this topic in recognition of the challenges. Significant progress has been made in abstracting the details from a three-dimensional model into a more simplified representation amenable to multiple realizations needed for a full probabilistic assessment. 


\subsubsection{Recommendations}

The workshop concluded with six key recommendations based on the presentations and discussions that took place:

1. There was a desire for some guidance on how to determine what level of complexity is needed and when there is a need to implement a probabilistic approach. It is not clear whether an a priori determination for these issues is generally possible. The general view was to maintain proper perspective that the PA process is intended to inform decisions and deterministic and probabilistic approaches should be used with that goal in mind. From this view, decisions regarding when to use deterministic and probabilistic approaches will be based on how much information is needed to make the decision at hand. It was recommended that part of an activity to develop some guidance could involve a review of existing positions regarding the use of deterministic and probabilistic methods (e.g., DOE-HS, NRC, EPA, international). A critical aspect of any recommendations will be an emphasis on the importance of conducting sensitivity or "importance" analyses and their use in the iterative assessment approach and the value that can be added by using a "hybrid" approach based on a combination of deterministic and probabilistic calculations.

2. The most significant technical recommendation was a clear need for additional guidance on the development of input distributions to be used for performance assessments. Experience to date has shown this is one of the most challenging aspects of moving to a probabilistic approach. The uniqueness of the problem for PAs entails a need to make modifications to traditional approaches. It was strongly recommended that a future workshop on this topic would probably be the best single area of focus resulting from this workshop.

3. The group believed it would be very beneficial to create a categorized data repository and relevant references from existing PA efforts that could serve as a resource for practitioners across the complex. Having this centralized resource would reduce time spent searching for data, reduce duplication of effort and also should help promote more consistency in approaches. The task should also be linked with similar activities for other organizations (e.g., ISCORS). A retrospective look at data and references used in existing PAs would be a first step towards building this resource.

4. Model abstraction was another technical issue that poses several challenges. As a first step, the group believed that it would be useful to summarize some of the benefits of having a simplified model available (e.g., ease of conducting special analyses, ease of explanation to stakeholders, QA of detailed model, composite analysis). This discussion could be included with a summary of some fundamental criteria to be considered when trying to develop a simplified representation of a more complex model. 
5. There is a need for some specific guidance on addressing uncertainties beyond variability (conceptual models, alternative exposure scenarios, multiple failure scenarios, etc.). There is a need for the ability to consider probabilities for some of these situations, especially in cases of highly unlikely what-if scenarios.

6. Two general topics were highlighted that need to be considered for all activities: benefits of involvement of reviewers early and often in the PA process and the need to maintain sufficient QA and documentation for the process, especially in the context of being able to transfer the work to others, if needed. 


\subsection{OPENING REMARKS}

Prior to the technical sessions, introductory remarks were provided from each of the sponsors of the workshop: DOE-HQ, DOE-SR, and the Savannah River National Laboratory. These introductory remarks were followed by an overview presentation introducing some key policy and technical considerations and summarizing the program for the workshop. This section includes a summary of the introductory remarks followed by a summary of key topics identified during the presentations and discussions. Table 1 includes a summary of the Rapporteurs and speakers for the workshop with titles for the presentations.

\subsection{INTRODUCTORY PRESENTATIONS}

Marty Letourneau, DOE HQ EM-11 sponsor for the workshop, provided introductory remarks (Figure 2). His remarks emphasized the importance of bringing the PA community together to share experiences as a means of fostering improved consistency. He noted that it had been a number of years since the PA community had been brought together like this. Bringing people together is especially important when the Complex is aggressively moving towards state-of-the-art approaches for conducting assessments. Along these lines, Marty also highlighted benefits of a "hybrid" assessment approach using both deterministic and probabilistic approaches for sensitivity and uncertainty analyses.

Howard Pope welcomed everyone on behalf of DOE Savannah River Operations Office and emphasized the timeliness of the workshop in the context of numerous PA and composite analysis efforts underway at Savannah River. He pointed out that a PA revision had just been approved for the E-Area disposal facility and that a composite analysis and three performance assessments are currently underway at the Savannah River Site (F Tank Farm, H Tank Farm, and Saltstone). These assessments are all expected to involve some probabilistic sensitivity and uncertainty analyses.

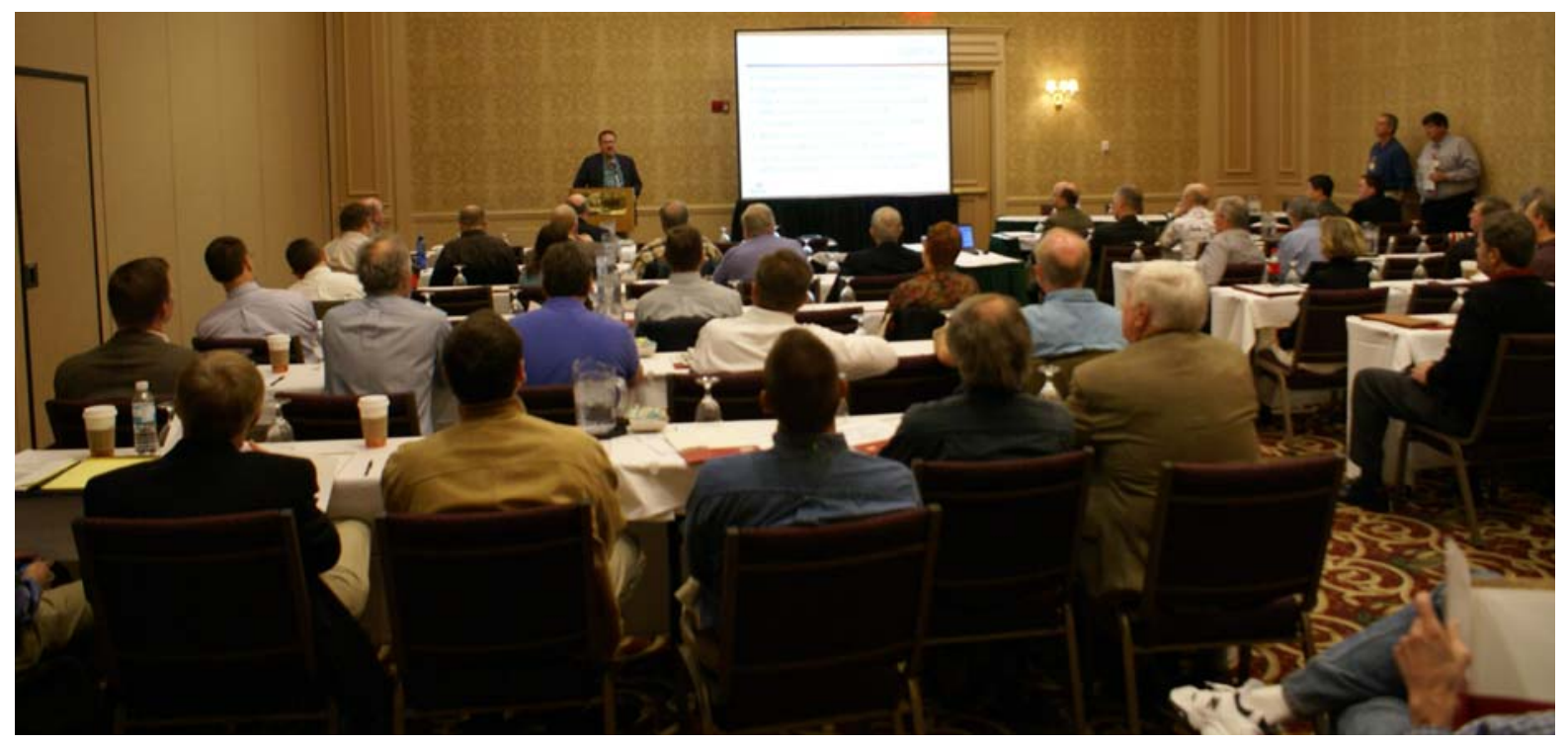

Figure 2. Martin Letourneau, EM-11 - Opening Remarks 
John Marra, Associate Laboratory Director for Environmental and Chemical Processing Technology at the Savannah River National Laboratory, welcomed everyone on behalf of SRNL and discussed how this activity is part of the variety of support being provided to DOE HQ as part of SRNL's role as the EM Corporate Laboratory. He emphasized the role of the National Laboratories supporting advances in assessment methods as well as the overall support for waste determinations and waste management for the DOE Complex as a whole.

Roger Seitz, Savannah River National Laboratory, provided an introductory presentation including some historical perspective on the evolution of the performance assessment process with an emphasis on the role of probabilistic approaches in the United States and Internationally. He discussed the concept of a hybrid approach that takes advantage of the strengths of deterministic and probabilistic calculations to improve the defensibility of an assessment and the concept of "importance analysis", a risk-informed sensitivity analysis focused on identification of parameters that have the most influence on the conclusion of the assessment (regulatory decision). The importance of keeping the performance assessment process focused on the decision to be made was emphasized.

\subsection{KEY POINTS}

Some of the key points made in the Opening Remarks include:

- Importance of bringing PA community together on a regular basis to share experiences and encourage communication as a means of fostering consistency,

- NRC supports increased use of probabilistic approaches for Tank Closure PAs and DOE sites are starting to use probabilistic approaches for LLW disposal facility Pas,

- Multiple performance assessments are underway and there is potential for inconsistent implementation of probabilistic approaches,

- Importance of maintaining perspective regarding the magnitude of performance measures relative to background and other radiation exposure limits,

- Use of assessment approaches that are focused on informing the decisions regarding compliance to avoid perception of PA as a modeling exercise,

- Recent experience demonstrating the utility of a "hybrid" approach for PAs taking advantage of benefits of deterministic and probabilistic methods, and

- Critical role of sensitivity analysis in the iterative approach to PAs and to focus reviewers' attention on key parameters and identify and justify key areas for future work and the use of the term "importance analysis" to highlight this decision-focused variation of sensitivity analysis used for PAs. 
WSRC-STI-2008-00333, REVISION 0

\subsection{SESSION 1: INPUT DATA AND DISTRIBUTIONS}

\subsection{INTRODUCTION AND BACKGROUND}

The opening session of the workshop focused on data development and translations to parameter input distributions used in probabilistic models. The last two decades has seen substantial progress in the development of credible models of flow and transport for a variety of hydrogeologic settings. The emphasis of model development continues to be on demonstrating credible model predictions which are numerical representations of the complex physical and chemical processes associated with the release, transport and exposure of radionuclides in the biosphere (process-based deterministic modeling). More recently there has been a paradigm shift from an emphasis on process modeling to augmentation of model predictions with quantification of the uncertainty of these predictions. The driver for augmented uncertainty assessment is for program and regulatory decisions (model-driven decision analysis); decision makers require increased information for effective decisions.

Probabilistic modeling where probability is the language of uncertainty is one of the essential tools used to quantify uncertainty. Probabilistic modeling includes three components:

1. Input parameters of the models are defined as probability density functions (PDFs; input distributions).

2. Monte Carlo simulation is used to repeatedly sample the parameter PDFs and each realization of random sampled values is propagated through a numerical model (process model, simplified process model or model abstraction; see the workshop summary for the session on model abstraction).

3. The model outputs of the multiple simulations are PDFs which provide a quantitative representation of the uncertainty of the model response.

Probabilistic modeling for performance assessments generally includes development of numerical simulation models of release, transport and exposure/dose conversions where input parameters are represented as PDFs. These modeling components are evaluated with sensitivity and uncertainty analysis using global probabilistic model outputs to identify key parameters affecting model uncertainty (importance analysis). The modeling and sensitivity analysis are linked with iterative cycles of data gathering, uncertainty reduction and measurement of the value of uncertainty reduction. Note that as modeling is more refined and details are added, uncertainty can increase in some cases. This suite of modeling approaches and methodologies can be described by different names including probabilistic risk assessment, quantitative risk assessment, uncertainty analysis, risk modeling and/or more simply, probabilistic modeling. 
The continuing growth of computer processing capability since the 1980 s aided by the recent use of multi-processor computer clusters with distributed processing now allows routine completion of complex numerical simulations for probabilistic modeling that would have been prohibitively time intensive a few years ago. However probabilistic modeling problems must still be carefully constructed to adequately address multi-components of uncertainty including variability, knowledge uncertainty, model uncertainty, conceptual or framework uncertainty and regulatory uncertainty (see Morgan and Henrion, 1990; Kammen and Hassenzahl, 1999; Cullen and Frey, 1999; Vose, 2000, Krupnic et al 2006, for background discussions of development of input distributions, definitions of uncertainty components and guidance on probabilistic modeling).

The state of the art of probabilistic modeling is evolving rapidly and the application of these modeling approaches to performance assessments for complex hydrogeological settings with spatially varying properties and multiple decision and regulatory end points is clearly still in development. Workshops like our Augusta meeting which bring together people with expertise in the wide ranging technical and policy disciplines required to develop competent performance assessments are clearly beneficial to the continuing development of performance assessments and the use of probabilistic modeling.

\subsection{PRESENTATIONS}

Bruce Crowe - NTS served as the Rapporteur for Session 1. Dr. Crowe has had a lead role in the implementation of probabilistic PAs for LLW in the DOE Complex. Session 1 included presentations by three speakers that bring unique perspectives and experience in the development and application of probabilistic models. Charley $\mathrm{Yu}$, of Argonne National Laboratory is the program manager for the RESRAD computer code and has considerable experience with the exposure/health physics disciplines of probabilistic modeling. David Esh is a senior systems performance analyst with the U. S. Nuclear Regulatory Commission with experience in regulatory evaluations of performance assessments and the use of the GoldSim simulation software. Paul Black is a president and co-founder of Neptune and Company with a background in Bayesian statistics and decision analysis and experience in developing, interpreting and applying the results of probabilistic modeling approaches to a variety of environmental problems.

\subsubsection{Yu Presentation}

Charley $\mathrm{Yu}$, during the first presentation on input distributions, provided background on probabilistic analysis implemented in the RESRAD code and contrasted differences between deterministic and probabilistic analysis. He summarized the continuous and discrete distributional forms available in RESRAD, described the distribution sampling methods implemented in the code, and provided cautions on using the built-in default distributions (preference for site-specific data). Charley provided examples and illustrated a critical data concept that must be considered in sampling probability distributions: correlation between input parameters. He concluded his presentation with an overview discussion of key issues identified through experience using the RESRAD code and developing and using probability distributions and interpreting the resulting model responses. 


\subsubsection{Esh Presentation}

David Esh emphasized the importance of properly utilizing data sources for constructing sitespecific input parameter distributions. He noted that sufficiency of data sources for input parameters is both difficult to obtain and expensive and he identified general sources of parameter data available through publications including performance assessments completed for DOE-regulated waste disposal sites. David supported the position of Charley $\mathrm{Yu}$, that the preferred data sources are local (site-specific) but noted that comparisons of site-specific input distributions with generic data can be useful. He emphasized the importance of establishing the representativeness of data and provided examples of required spatial and temporal averaging needed to properly interpret data for translation into input distributions. Challenges associated with correlation were also identified and discussed.

David described the importance of a risk-informed perspective including iterative cycles of data selection and implementation and examination of model response. He concluded by urging the performance assessment community to consider the advantages of establishing databases of technical information as a resource for probabilistic modeling (for example, a Wikipedia performance assessment site).

\subsubsection{Black Presentation}

Paul Black presented decision contexts for probabilistic modeling and the development stages needed in model preparation, model structure and model specification to produce adequate model response, where adequate reflects physically plausible models with proper representation of uncertainty. He noted the importance of averaging parameter inputs over the space and time scales of the modeling problem so that model response represents system uncertainty and not variability (i.e. the model output is the distribution of an average). Paul summarized the information sources used for developing input distributions including site data, metadata, model abstraction and elicitation. He provided examples from performance assessments representing alterative sources of data. He also described examples where distribution forms for input data do not provide expected model responses, underscoring the need to always examine model results for appropriateness.

Paul described examples of distribution forms (uniform, triangular) that may not provide physically realistic sampled data distributions. He noted, consistent with the previous two speakers, the issues of data correlation particularly for multivariate parameter data. He urged performance assessors to work with statisticians who are unafraid of messy environmental data sets and to always ensure input distributions can withstand statistical rigor, are adequately documented and have been peer reviewed. Not following these common sense constraints can compromise model response and sensitivity and uncertainty analyses of model output. Paul argued that probabilistic-based modeling can be used as a decision tool for multiple components of performance assessment including compliance, future disposal decisions, maintenance and operations, and closure and monitoring. 


\subsection{DISCUSSION}

The wrap-up discussions for the input distributions session of the workshop produced lively and thought-provoking comments from the audience. A number of comments emphasized the importance of retrospective reviews of existing waste management performance assessments to benefit from the experiences gained from completing and obtaining review evaluations of the work. Post-audit reviews of model outputs from probabilistic models were noted as important for ensuring credibility of model results - propagation of conservatism in probabilistic modeling can lead to physically unrealistic model outputs.

A range of opinions were expressed on the need for and requirements for development of probabilistic input distributions for dose conversion factors, scenario parameters and human habit components of performance assessments; there was not agreement that all of these model components required probabilistic parameter inputs. This was discussed further in the policy session. Multiple questioners were interested in documentation of clear examples and demonstrated advantages of the application of probabilistic modeling. The discussion highlighted a range of opinions on PA modeling and the perspective that many people maintain preferences for fully deterministic or fully probabilistic approaches.

Comments were expressed about the existence of guidance for developing probability density functions for model development (see cited references and software manuals developed for probabilistic modeling platforms, for example, GoldSim, Analytica, @Risk and RESRAD where the manuals can be downloaded from web sites). There was a consensus that development of defensible (transparent) distributions is one of the most significant challenges in moving to a probabilistic approach. Another policy issue that was passed forward was the need for criteria on which to judge compliance for probabilistic assessments in the face of a deterministic standard. This is also discussed in the policy session (Section 6).

A variety of comments expressed healthy skepticism over the value of probabilistic modeling particularly where the models are based on simplified model abstractions and, likewise, healthy skepticism about the value of deterministic calculations that do not adequately represent uncertainty. The use of simplified model abstractions is discussed in detail in Section 4.

Several people mentioned the potential benefits of a task to assemble lessons learned from disposal sites that have completed and/or are continuing development of probabilistic performance assessments models (Nevada Site Office for the Area 3 and 5 disposal facilities; Idaho Site Radioactive Waste Management Complex; Los National Laboratory for the MDA G site, and the Savannah River Site for the E-Area disposal facility and the F-Tank Farm as well as new assessments for H Tank Farm, Saltstone and the composite analysis update). 


\subsection{RECOMMENDATIONS}

Several common themes were apparent through the presentations and discussions. The difficulties and importance associated with establishing and clearly justifying input distributions from limited characterization data was clear to all participants. There was ample discussion regarding the benefits, difficulties and costs associated with implementing a probabilistic approach and the need to weigh the difficulties against the benefits. It was clear that healthy skepticism needs to be maintained regarding all modeling approaches.

One recommendation that received support was to take a retrospective look at existing PAs both as a source of data and associated parameter distributions as well as references for additional information that may be useful for other PAs. There was strong support for development of a common data resource that would serve as a repository for data that have been used in PAs. A Wikipedia type approach was suggested as an example for building PA resources.

There was a consensus that there is an immediate need for guidance on the development of parameter distributions, including when to pursue elicitation and also with respect to the choice of distribution forms, methods used for spatial and temporal averaging and approaches used to assess parameter correlations. It was recommended that the next PA modeling workshop should emphasize this topic.

The importance of having site-specific data was strongly emphasized, but value was recognized in evaluating parameter distribution data from existing PAs as a starting point. There is a tendency to start simple with more general distributions (uniform, triangular), but several people highlighted the importance of recognizing the impact this can have on both the skewness of the output distribution and representation of the tails of the distributions. It was recommended to emphasize the importance of trying to develop parameter distributions that represent average parameter values appropriate to the temporal and spatial scales of sitespecific performance assessment. This reflects the ability to move more towards realistic bias rather than conservative bias through the use of probabilistic approaches.

The group was essentially unanimous in recommending the use of a risk informed approach in an iterative manner to refine key assumptions and parameter distributions based on the results of sensitivity and uncertainty analyses. This philosophy applies for both deterministic and probabilistic approaches. 
WSRC-STI-2008-00333, REVISION 0

This page intentionally left blank.

$-18-$ 


\subsection{SESSION 2 MODEL ABSTRACTION}

\subsection{INTRODUCTION}

Estimating dose from the groundwater pathway for an LLW performance assessment often requires modeling flow and transport in a complex hydrogeologic setting. For many sites the complexity of the groundwater model developed to represent site behavior can conflict with the need for a model sufficiently simple to allow simulations required to quantify model uncertainty. Beginning the model development process with fully 3-dimensional (3-D) transient conceptual-mathematical models of the hydrogeologic system is recommended by Neuman and Wierenga (2003). If model simplification can be justified, these authors suggest approaches including:

- reduced dimensionality (spatial and/or temporal)

- reduced model size

- reduced details of various features, events, and processes

- reduced detail in representing internal heterogeneity

- single continuum rather than dual continuum or discrete fracture representation

- simplified surface boundary conditions

- reduced detail in representing contaminant sources

- constant rather than scale-dependent dispersion

- equilibrium rather than kinetic sorption

Neuman and Wierenga (2003) argue that the model simplification process must be systematic and objective, providing a formal demonstration that the simplified model has the detail and capabilities to represent the site's essential features and factors as well as a more complex 3-D transient model. This is an advantage of the "hybrid" approach that blends the use of detailed deterministic models with more simplified representations that are more amenable to fully probabilistic calculations.

\subsection{PRESENTATIONS}

Mike Sully - Neptune and Company served as the Rapporteur for Session 2. Dr. Sully offers a broad range of experience and perspective spanning academic, research and applied work on hydrogeology, including involvement in the development and reviews of DOE Complex LLW performance assessments. The first presentation was provided by Greg Flach and Glenn Taylor of Savannah River National Laboratory. Dr. Flach and Mr. Taylor were the key contributors to the development of the links between the 3-D PORFLOW ${ }^{\mathrm{TM}}$ and simplified GoldSim representations used in the modeling for the F Tank Farm performance assessment at Savannah River. 
The second speaker was Phil Stauffer of Los Alamos National Laboratory. Dr. Stauffer was the lead for developing the abstraction approach used to link the 3-D FEHM and GoldSim representations for the Area $G$ performance assessment at Los Alamos. The third speaker was Matt Kozak of Monitor Scientific. Dr. Kozak was one of the key players in the development of the original NRC work related to application of probabilistic approaches for LLW disposal PAs and also offers many years of international experience in the field.

\subsubsection{Flach and Taylor Presentation}

Greg Flach and Glenn Taylor described their model abstraction approach for the F-Tank Farm Performance Assessment at the Savannah River Site. The need for a simplified model was motivated by the need to address the significant uncertainty in failure scenarios and input data. A detailed model for the tanks consisting of three parts: closure cap infiltration, vadose zone flow and transport, and aquifer flow and transport was developed. The approach to simplification followed from recognizing that some complexities of the system may not be relevant for all scenarios (Pachepsky et al. 2006). Two primary performance analysis scenarios were selected for modeling. In the first, the system behaves as intended characterized by slow, gradual material degradation. In the second, the steel liner fails early and infiltration is focused on the source zone. Detailed simulations were conducted with a 2-dimensional (2-D) PORFLOW ${ }^{\mathrm{TM}}$ (ACRI 2004) vadose zone model demonstrating that prior to liner failure while the flow field was multi-dimensional, there is no impact from reducing the dimensionality of the flow field because there is no contaminant release. Following liner failure the simulations demonstrated that the flow field was approximately 1-dimensional (1-D) allowing a simplified model of reduced dimensionality to be developed.

For the saturated zone the flow field was represented as a collection of 1-D streamtubes calculated using a saturated zone PORFLOW ${ }^{\mathrm{TM}}$ model. These 1-D representations of the flow fields provided water fluxes to a simplified 1-D GoldSim (GoldSim 2007a, 2007b) radionuclide transport model. Radionuclide breakthrough curves estimated using the GoldSim model were compared with breakthrough curves estimated with the PORFLOW ${ }^{\mathrm{TM}}$ models to identify differences between the models. Discretization of the GoldSim model was increased to minimize differences between the breakthrough curves and model comparisons. As these refinements were added to the GoldSim model, it led to insight into processes in the PORFLOW ${ }^{\mathrm{TM}}$ code not previously considered, which is an advantage of looking at the problem two different ways.

\subsubsection{Stauffer Presentation}

Phil Stauffer described the simplification of a groundwater flow and transport model for the MDA G Performance Assessment at the Los Alamos National Laboratory. This model simplification was motivated by the need to link a flow and transport model in a complex hydrogeologic setting with a probabilistic performance assessment model. A detailed 3-D flow and transport model incorporating previous modeling and available data was developed using FEHM (Zyvoloski et al. 1997a, 1997b; Dash et al. 1997). Simulations were conducted to examine system behavior with the 3-D model to identify less important features and factors. A systematic abstraction process was followed to represent 3-D pathways with 1-D pathways. With this process, dimensionality of the model was reduced while still capturing essential features of the complex system. 
Micromixing theory was used to include decay, ingrowth, and sorption along the pathway and a water well abstraction was developed to quantify the capture efficiency of the water well. Radionuclide breakthrough curves calculated with the simplified model were compared with curves calculated with the detailed 3-D model to verify that the simplified model provided an adequate representation of the system. This simplified model was linked to the GoldSim performance assessment model to provide calculations of the mass flux of radionuclides from the groundwater pathway. In this model GoldSim samples distributions of the sorption coefficient, the infiltration rate, and the inventory and passes the information to the simplified FEHM model. The FEHM model calculates the mass flux of radionuclides at the receptor location and returns the information to GoldSim. This simplification of the groundwater pathway model allowed for Monte Carlo simulations to be conducted that were adequate to support uncertainty and sensitivity analyses.

\subsubsection{Kozak Presentation}

Matt Kozak addressed model abstraction and the treatment of model uncertainty. The overall structure of uncertainty or importance analysis was introduced highlighting the differences between deterministic and Monte Carlo analyses. It was emphasized that full treatment of uncertainty, however, requires more than a Monte Carlo analysis of uncertain parameters. The use of alternative conceptual models developed using a broad base of expertise allows for the consideration of broader uncertainties. Alternative conceptual models arise from differing assessment contexts, differing scenario definitions, and evaluations using exploratory conceptual models. Kozak defines the concept of performance margin analysis as the use of probabilistic modeling to evaluate model assumptions or conceptualizations that are plausible, but not the most likely. These analyses can provide some quantitative estimate of the conservatism of other models.

A case study involving volcanism at Yucca Mountain was presented as an example of performance margin analysis. A second case study for a site at Vaalputs, South Africa demonstrated the benefits of considering alternative conceptual models. Addressing model abstraction through model intercomparison was proposed as a primary tool for producing credibility.

\subsection{DISCUSSION}

The discussions raised the issue of how to determine when a system can be simplified. Modelers observed that technical challenges to model abstraction included multi-dimensional phenomena, the influence of sub-site scale features and larger scale features on site-scale flow and transport phenomena, non-linear or chaotic behavior, and competing effects and mechanisms. Thus, a system characterized by approximately 1-D phenomena, insensitivity to sub-site scale features, linear behavior, and clearly defined and easily simplified driving mechanisms may be appropriate for simplification. Since the model objectives can influence both the need for and the approach to simplification, the objectives of the modeling must be clearly identified. 
It was recognized that simplification of models has some risk in the context of defensibility. A number of people mentioned that having two separate representations of a problem offered benefits for both the detailed and the simplified model. This utility was demonstrated for the F Tank Farm PA, where both the detailed model and the simplified model were improved as a result of the abstraction effort. There was support for the idea that, in order to justify the potential compromises that are often necessary to abstract a simplified representation, it is important to identify the benefits of having a simplified representation, for example:

- Computational efficiency, which facilitates the ability to make multiple runs,

- Accessibility and relative ease of explanation to stakeholders

- Benefits of complementary models to build confidence

- Quality Assurance checks of the detailed model

- Ability to obtain relatively quick responses for Special Analyses needed to justify disposal of unusual waste streams

The question of identifying when a deterministic model was adequate was discussed at length with a range of differing opinions expressed. There was a view that informed use of deterministic models and limited uncertainty analyses may actually be as good or better than trying to develop a simplified model and then develop input distributions, etc. In this case, the emphasis is on the skill of the analyst rather than using probabilistic analyses as a means to gain that knowledge. This was a controversial topic. In addressing the question of what degree of model refinement is adequate for a give regulatory purpose, Neuman and Wierenga (2003) maintain that decisions such as this are in the domain of economic and policy concerns and are ultimately the responsibility of managers and decision-makers to resolve. Once again, this highlights the importance of recognizing the role of PA as a decision making tool, not a decision-maker. Similarly, there was debate as to how to weight alternative models or scenarios in the context of a regulatory decision. There was also general agreement that some comparison of the model with plumes of existing contamination at a site is desirable as a confidence building tool.

Workshop discussions identified three choices for modeling approaches to quantify uncertainty.

The first approach was that described in both case studies presented. Multiple iterations of deterministic process models are run to define permissive ranges of system output for abstraction into simplified models. This approach is heavily dependent on the quality and quantity of deterministic model runs to adequately characterize system response. The abstracted model can then be run via Monte Carlo simulations to produce probabilistic model outputs. The abstraction converts process response into uncertainty response and the Monte Carlo simulations must be carefully constructed to ensure that the sampled parameter ranges remain within the physically plausible range of the process domain. While this approach is less computationally demanding it requires significant process justification from the deterministic modeling effort. 
A second approach uses high-performance computing to provide a sufficient number of simulations of a complex model within a practical schedule. Distributed computing using large multi-processor clusters is used to provide sufficient processing power to run deterministic flow and transport codes in multiple simulation cycles with parameter lookup tables that allow for stochastic sampling with a sufficient number of iterations to provide realistic probabilistic model output. This is the most appealing approach from a process perspective but it comes at a very high computation price.

The third approach, developed by Los Alamos National Laboratory, is being used to simulate the concentration of plumes originating from the locations of underground nuclear tests at the Nevada Test Site. This is a reduced dimensionality approach in which multiple alternative flow models are developed for calibration of the flow field. These models provide boundary conditions and fluxes for multiple iterations of transport codes. The 3-D transport simulations using FEHM produce time histories of spatial locations and residence time distributions of particles introduced at source locations. A convolution based particle tracking (CBPT) method is used for efficient Monte Carlo simulations with an arbitrary number of time dependent functions describing release from the sources and stochastic sampling of transport parameters. This approach requires development of multiple conceptual models of the flow field to define a suite of permissible flux and boundary conditions for the 3-D transport simulations and repeated cycles of Monte Carlo simulations by radionuclide for developing model outputs that are converted to CDFs of groundwater concentration. The CBPT method is valid for steady state flow fields and linear transport processes. This approach is also computationally demanding but less so than the previous option, though likely to require distributed processing capability.

\subsection{RECOMMENDATIONS}

Retrospective reviews of existing PAs were proposed as a means to collect information on the development of alternative conceptual models and approaches to model simplification at other sites. The reviews would determine if simplifications were made, how these simplifications were implemented, and what criteria were used to evaluate the validity of the simplified models.

There was also a recommendation for the preparation of a brief summary of some of the benefits of developing simplified models. This is linked back to the issue that simplification will involve additional effort and some compromises, so there needs to be a good reason for abstraction. Some examples of the potential benefits were identified in Section 5.3. 
Approaches to model simplification for the PAs discussed in the Workshop were developed using the best judgment of the modelers, however, discussion drew attention to the fact that the group was not aware of an accepted structured, systematic approach to model simplification/abstraction. A formalized process for model abstraction was proposed by Pachepsky et al. (2006). His recommended steps are:

1. Justify the need for the model abstraction,

2. Define the context of the modeling problem,

3. Select applicable model abstraction techniques,

4. Choose which techniques will result in the greatest benefits, and

5. Simplify the model by applying those techniques.

While these steps describe a simplification procedure, they do not address the question of how to demonstrate that the simplified model adequately captures the essential features of the hydrogeology and flow and transport dynamics of the more complex model. Emphasis was placed on the need for this to remain a somewhat subjective process that will need to be a focus of reviewers rather than subject to some specific numerical or statistical criteria. Some fundamental guidelines for evaluating the adequacy of the abstraction are nevertheless needed.

Model intercomparison of simplified and complex models and alternative conceptual models was recommended to enable a better understanding of the system and to provide a quality assurance check for all models. The complex and simplified models can be complementary, together offering a more complete view of the flow and transport dynamics. 


\subsection{SESSION 3: IMPLEMENTATION OF SENSITIVITY AND UNCERTAINTY ANALYSES}

\subsection{INTRODUCTION}

Two key pieces of a probabilistic sensitivity and uncertainty analysis were considered in Sessions 1 and 2. In the third session construction of a complete analysis was explored which is not necessarily completely probabilistic. Topics from the first two sessions continued to be discussed but in the context of the analytical approach, analysis goals and applications. Other topics discussed included:

- general concepts for constructing and completing sensitivity and uncertainty analyses with some examples provided, and applications of analytical results to decision making,

- techniques for manipulating model input data to generate uncertainty estimates for the model outcome and estimate the sensitivity of the model outcome to various parameters,

- the relative merits of deterministic versus probabilistic approaches for estimating outcome uncertainty and system sensitivities,

- interpretation of probabilistic results relative to an understanding of system dynamics (e.g., cause and effect relationships), and

- ease of communicating the results and implications for decision making to the regulator.

\subsection{PRESENTATIONS}

Marc Wood - Fluor Hanford served as the Rapporteur for Session 3. Dr. Wood has been involved in performance assessments for more than 25 years and offers broad perspective from experience on assessments for Basalt Waste Isolation Project HLW repository, LLW disposal facilities and Tank Closure assessments. The first presentation was provided by Art Rood of the Idaho National Laboratory. Mr. Rood was a key contributor to one of the first assessments in DOE to successfully use a combination of deterministic and probabilistic calculations to build a PA for the Radioactive Waste Management Complex at the INL Site. The second presentation was given by Paul Black of Neptune and Company to provide fundamental statistical background regarding sensitivity and uncertainty analysis. The third presentation was provided by John Tauxe of Neptune and Company. Dr. Tauxe has been involved in model development and application for the implementation of probabilistic approaches for performance assessments at DOE sites. 


\subsubsection{Rood Presentation}

Art Rood described a sensitivity and uncertainty analysis for LLW disposal at the RWMC. A critical first step was to define the assessment question to help focus on the relevant sources of uncertainty and to evaluate those system aspects for which the analysis outcome was most sensitive. In this example, uncertainties from natural and engineered barrier conditions affecting future contamination were considered whereas exposure scenario factors were not. To begin the analysis a base case was completed deterministically to identify dominant groundwater contaminating radionuclides. Then a limited set of one factor at a time (OFAT) analyses were completed in which those parameters considered most significant to the estimated future groundwater contamination were varied individually with respect to the base case analysis. These analyses provided some comparison with results from subsequent probabilistic uncertainty and sensitivity analyses.

The remainder of the analysis consisted of probabilistic analyses aimed at providing two products. The first product was an envelope of outcomes (groundwater contamination levels) around the deterministic result that reflected uncertainty in the quantification of significant processes and features which translated to uncertainty in the future groundwater contamination estimates. The range of results varied as a function of time because dominant parameters with differing probability density functions changed over time. The second product was a set of sensitivity analyses that quantified relative significance of various parameters with respect to their influence on future levels of groundwater contamination. A rank correlation technique was used to develop this information. As expected, the significant parameters changed over time because of variable recharge history imposed by engineered cap placement and variable radionuclide-specific chemical reactivity. For both products, a larger set of parameters were considered with each parameter being assigned a probability density function that was determined from both empirical data and subjective judgment of subject matter experts. The transport model was then run numerous times (250-500 realizations were considered adequate) in a Monte Carlo simulation in which parameter values for multiple parameters were selected randomly.

Rood concluded that the collection of results provided a sense of the precision or range of outcomes but did not necessarily define the accuracy of the results (e.g., the central tendency result may or may not have been determined). This occurs because results were dependent on the reliability of parameter selection and assigned values (e.g., probability density functions). Consequently, model validation in some other form is desirable. Despite this caveat, the results were still considered useful for comparison with performance objectives and determination of adequate facility performance. 


\subsubsection{Black Presentation}

Paul Black focused his discussion on the components of sensitivity and uncertainty analyses needed to make decisions that are at least partially dependent on analysis outcomes. With regard to PA models that are used to determine compliance with performance objectives, several aspects are important. First, uncertainty analyses should evaluate environmental contamination over time. Second, sensitivity analyses must focus on realistic conceptual models and expected parameter values to avoid meaningless results. Third, sufficient realizations must be done to ensure that minimum uncertainty has been calculated. Fourth, work remains to better clarify the meaning of acceptable levels of uncertainty for decision making.

Sensitivity analyses must be constructed for conditions that are multidimensional, often non linear and non monotonic. In such systems, parameter variability effects on the analysis outcome (e.g., radionuclide groundwater contamination at a downstream well) can be looked at in isolation (one factor at a time analysis or local sensitivity), but must also be looked at in the context of other parameters (global sensitivity) to determine the influence of factors simultaneously affecting the outcome. In this way, the most complete understanding of primary processes and features affecting the analysis outcome can be determined. In addition, some apportionment among parameter variability effects on outcome variability is viewed as desirable to direct future efforts at reducing overall uncertainty and thereby improving confidence in the decision.

A central proposition was that the most comprehensive analysis is best done probabilistically. However, such an approach is likely to be computationally intensive. Therefore, other desirable features of such an analysis are efficiency, simplicity and the ability to handle nonlinearity. Fortunately, advances in hardware and software computational capabilities have enabled extensive comparisons of multiple parameter effects and interactions. In particular, data mining and machine learning approaches appear to be among the most flexible and capable. Examples of parameter rankings from the Los Alamos National Laboratory (LANL) low level waste burial ground analysis and Nevada Test Site (NTS) were given. Significant results from these analyses were a determination of significant parameters and a quantitative estimate of their impacts on the variability of analysis outcome (environmental contamination). Also, one application of the probabilistic analysis to focus additional sitespecific data gathering was provided. A subsequent analysis was then shown to have reduced calculated uncertainty because of new data input. 


\subsubsection{Tauxe Presentation}

John Tauxe presented approaches for conducting sensitivity and uncertainty analyses for different types of uncertainty related to conceptual models, mathematical models and input parameters. Conceptual model uncertainties arise when attempting to truly represent and simplify natural (e.g., hydrogeology) or engineered (e.g., infiltration) features and processes for translation into numerical models. Alternative representations of features or processes are a typical way to consider conceptual model uncertainties. Mathematical model uncertainties arise when various mathematical representations of a process can be used and the supporting database is unable to indicate the best choice. An example was given from the NTS PA analysis where various air phase tortuosity equations were considered. The uncertainty analysis to evaluate the impact of using different equations to estimate radon flux showed that variability of flux estimates were sensitive to the equation selected. Parameter uncertainties result from basic understanding of their effects on the outcome and natural variability induced by system heterogeneities. These uncertainties are expressed through selection of distribution functions which take numerous forms (e.g., normal, log normal, triangular, uniform) and are selected largely on the basis of available supporting information and expert judgment.

Once the pertinent representations of various kinds of uncertainty are made, many simulations (or realizations) are performed in which parameter values are randomly selected from the probability density function data set, a suite of realizations are developed to envelope a range of possible outcomes and predict a central tendency. Additional examples of parameter sensitivity analyses for a generic subsurface flow and transport analysis were also provided, illustrating the uses of the data mining and machine learning data manipulation techniques also described in the previous paper. 


\subsection{DISCUSSION}

Workshop participants generally agreed that a process exists for conducting sensitivity and uncertainty analyses. The process begins with the definition of a problem to be solved. In this workshop the problem was to determine the adequacy of leaving waste in the near surface, either as a deliberate disposal action or the end result of a remediation effort. To make this decision, reliable determination of effects due to waste release from a disposal facility must be developed. Implicit in the task is a need to adequately describe the range of plausible outcomes that arise from a limited understanding of system behavior and inherent process and feature variability. The second step involves construction of the modeling framework and establishment of parameter input data, determination of outcome uncertainty and sensitivities of the outcomes to particular site-specific processes.

The importance of using an iterative approach was emphasized, where the sensitivity analysis results are used to identify data or distributions that need to be refined and justify work to refine those data. The updated data are then used in the next iteration of the analysis. This is an essential aspect of a cost-effective approach to PAs. There was also a lot of discussion regarding the role of a robust sensitivity analysis as part of a demonstration that the system dynamics are relatively well understood. This demonstration of understanding of the system behavior is seen as a critical element for building confidence that the uncertainty analysis, and the PA in general, are reliable and sufficient for use in decision-making,

There was general agreement that a deterministic base case analysis is required as a first step in the process and that the analysis should be sufficiently complex to capture the most important system features and processes affecting the outcome (e.g., levels of environmental contamination over time versus standards for such contamination). The next step in the process, representation of outcome uncertainty and determination of key parameters and relative impacts, is more difficult. Probabilistic approaches were discussed primarily, but deterministic approaches were also considered. In the case of probabilistic approaches and development of probabilistic input distributions, the importance of focusing on average behavior was emphasized. The participants generally agreed that probabilistic approaches had numerous advantages and had strong support among some regulators, particularly the NRC. For ongoing and future DOE actions involving review by the NRC (e.g., waste determinations for tank waste), significant reliance on probabilistic approaches is assumed.

The advantages and disadvantages of both probabilistic and deterministic approaches were discussed. Workshop participants agreed that probabilistic techniques coupled with simplified transport models and current computational capability clearly permit more efficient data manipulation for evaluating uncertainty and sensitivity effects. Arguments were also made that probabilistic approaches provide a means to move towards more realistic representations rather than having to build-in conservative bias as is sometimes the case for deterministic approaches. Therefore, far greater sampling of parameter input values is available and many more outcome calculations are feasible. 
At the same time, the probabilistic analysts warned that the enhanced computational capacity should be used judiciously and to avoid incorporation of unlikely conditions into the analysis structure which would distort uncertainty and sensitivity estimates. Also, depending on the problem being addressed, certain parameters could be excluded from the uncertainty and sensitivity evaluations. For example, there was general agreement not to consider uncertainties and sensitivities associated with dose methodology when considering translation of environmental contamination levels to human exposure.

Deterministic techniques are less efficient to represent uncertainties and typically, a limited set of outcomes can be calculated. Those comfortable with deterministic analyses proposed that some deterministic sensitivity and uncertainty analyses were viable and useful when there was a relatively good understanding of parameter effects on the outcome. Also, relating sensitivity and uncertainty results to cause and effect relationships in the analyzed system may be more straightforward with the deterministic approach. The concept was put forth that a suite of deterministic analyses, properly constructed, may achieve or partially achieve the same goals of a more comprehensive probabilistic analysis. That is, a range of results that bound the expected outcomes could be derived and those parameters strongly affecting outcome uncertainty could be determined. Recognizing that strengths and weaknesses exist for both approaches, there was general agreement that use of both approaches to complete sensitivity and uncertainty analyses would have value.

For those in the audience inexperienced in the practice of probabilistic analysis, several concerns and concepts not well understood were raised, some of which had been expressed in previous sessions. These included the following:

- completeness and reliability of data from which distribution functions are derived, particularly when a rigorous statistical database is rarely available to construct probability density functions,

- validity of simplified models needed to facilitate the generation of many realizations,

- apparent focus of probabilistic analyses on parameter variability and limited experience with treatment of alternate conceptual and mathematical models,

- difficulty in clearly linking analytical results to significant processes and features driving environmental outcome,

- the distinction between the overall model sensitivity versus importance of various parameters to the specific decision to be made and how the analyses are used to make this distinction (e.g., separate sensitivity analyses for different peaks in the results),

- influence of variability in the types of assumed probability distributions for a given parameter with respect to estimated outcome uncertainty, and

- potential difficulty in clearly explaining the meaning and application of probabilistic results to a decision maker unfamiliar with these analytical approaches. 
Finally, some commonality in results appears to exist among the disposal conditions evaluated. First, uncertainty estimates and dominant parameters tend to change as a function of time in groundwater pathway analyses. Two aspects of the disposal condition likely contribute to this observation, one being a variable recharge rate history induced by infiltration cap placement, and the other being variable chemical reactivity among radionuclides with subsurface soils. Therefore it is important to carry analyses over a long period of time. Second, in the cases presented, a small number of parameters (and therefore a small number of features and processes represented by those parameters) appear to contribute most to uncertainty and sensitivity results at any given time. These results support general intuition about which features and processes should be controlling waste migration (e.g., recharge history, geohydrology, sorption).

\subsection{RECOMMENDATIONS}

Among those attending the workshop, a large range of expertise and experience with conducting probabilistic sensitivity and uncertainty analyses was present. If probabilistic analyses are going to become widespread in the DOE system, some additional education for the current non practitioners is warranted. More detailed workshops on particular aspects of the probabilistic analysis would help the education process (e.g., future seminars on the construction of probability density functions for specific waste disposal or remediation conditions). A continuing dialogue is suggested relating to the suitability of probabilistic versus deterministic approaches for the type of problem being considered. For example, if compliant disposal with performance requirements is easily demonstrated because of system conditions, how important is quantification of uncertainty with probabilistic analyses? Also, continued development of criteria that help judge the reliability of sensitivity and uncertainty analyses, regardless of approach taken, is beneficial.

Experience with probabilistic assessments results in several recommendations that are key to conducting a successful analysis:

- Assessment needs to be focused on a clear question to be answered (avoid tendency to become a modeling exercise rather than a problem solver),

- Role of a robust sensitivity analysis as part of a demonstration that the system dynamics are relatively well understood, thereby increasing confidence that the uncertainty analysis, and the PA in general, are reliable and sufficient for use in decision-making,

- Focus on average behavior. Inclusion of unlikely behavior (largely a qualitative judgment) in a probabilistic analysis distorts the calculated outcomes and compromises the applicability of the results for decisions making (compliance, closure actions, etc.),

- Document the basis for probability density functions extensively. Concurrence with these selections is critical to a believable analysis, and

- Conduct sensitivity analyses at different times to evaluate how the relative importance of input parameters can change depending on the time of the analysis (i.e., multiple peaks typically occur in PA results and the different peaks will often depend on different inputs). 
Several specific recommendations for future work were identified over the course of the discussions:

- Need for guidance on fundamental questions (e.g., when deterministic analyses are enough to demonstrate compliance and how many cases need to be considered, need for probabilistic screening, how to present low probability scenarios, potential to use probabilistic approach as confidence building tool rather than for demonstration of compliance),

- Need for guidance on conduct of sensitivity analyses for non-linear systems when basic rank correlation approaches may not be sufficient, and

- How to know when an abstracted model is sufficient for sensitivity analysis (is the simplified model sufficient to identify the key parameters controlling behavior?). 
WSRC-STI-2008-00333, REVISION 0

\subsection{SESSION 4: POLICY IMPLICATIONS}

\subsection{INTRODUCTION}

The policy session included two informal presentations that largely reflected on discussions in the previous sessions in the context of DOE policies and initiatives. The intent of the session was to provide some reality check to identify practical limitations that may exist at a policy level. Two informal presentations were provided that gave perspective from the LFRG and also from the health and safety side of DOE-HQ.

\subsection{PRESENTATIONS}

Roger Seitz - SRNL served as Rapporteur for Session 4 on Policy Implications, which was an informal discussion of policy issues. Roger has more than 20 years of experience on PAs at multiple DOE sites and around the world. The first presentation was from Howard Pope, the DOE-SR representative on the LFRG. Howard highlighted a few of the issues that the LFRG is wrestling with related to moving towards probabilistic approaches. The issues that have been a concern with the LFRG were generally consistent with many of the questions and discussions that had taken place in the earlier sessions, for example:

- identification of point of compliance and representation in a probabilistic assessment,

- use of peak of means, mean of peaks, median, etc. as the compliance determination with a deterministic standard,

- how to consider probabilistic results other than the central tendency in a compliance determination (e.g., $95^{\text {th }}$ percentile),

- how to address low probability scenarios, and

- how to address results that may exceed the standard after the time of compliance.

The second presentation was provided by Andy Wallo, DOE-HQ HS-20. Andy began with some links to the application of probabilistic risk assessment (PRA) in the safety analysis world and Defense Nuclear Facilities Safety Board (DNFSB) positions. DOE-HS is preparing a position paper on the appropriate use of PRA in safety analysis. Andy believed that for safety analysis and performance assessment, probabilistic assessment needs to be seen as a tool to help support decision-making rather than a required process. The need for conservative deterministic calculations is still recognized in the safety analysis community.

Andy discussed the activity to incorporate probabilistic methods in RESRAD and how HS (formerly EH) have been developing the capability to conduct probabilistic calculations for a number of years. Andy discussed concerns regarding knowing when deterministic approaches are enough and how to address probabilistic results in the context of deterministic standards. Andy concluded by re-emphasizing the importance of recognizing the purpose of a deterministic and/or a probabilistic assessment is to help make sound decisions and the type of approach used should be selected in the context of helping to make a sound decision. 


\subsection{DISCUSSION}

There was lively discussion with a variety of different opinions regarding the policy issues, which are linked to many of the issues discussed previously. The topic of interpretation of probabilistic results in the context of deterministic standards generated a lot of discussion with opinions varying from the idea that well thought out deterministic approaches are sufficient all the way to a need to establish limits on the tails of distributions like the $95^{\text {th }}$ percentile in addition to the central tendency when using probabilistic approaches. The topic of how to handle cases where the $25 \mathrm{mrem} / \mathrm{yr}$ standard is exceeded at times beyond the time of compliance was also discussed. The general feeling was those cases should be dealt with in the context of a decision making framework on a case-by-case basis (i.e., an exceedence beyond the time of compliance does not trigger a non-compliance).

The discussion also included a number of opinions regarding how to handle low probability what-if cases in a probabilistic framework. Traditionally, one of the benefits of a probabilistic approach is the ability to assign a probability to such cases and either screen them from consideration for very low probabilities or implicitly include the probability in the calculation. The NRC Staff have recommended that a probability of one be assumed for intruder scenarios as part of accepted practice, while DOE Order 435.1 has indicated that it is possible to take credit for probabilities of intrusion with appropriate justification. However, the question here goes beyond intrusion and is related to what-if type cases for alternative conceptual models, etc. for the all pathways scenarios. The ability to take account of the relative likelihood of different what-if cases reflects the advantage of moving towards more realistic interpretations as part of a probabilistic approach.

There were also discussions regarding how to conduct screening and develop waste concentration or inventory limits within a probabilistic framework.

\subsection{RECOMMENDATIONS}

The group agreed that there are technical and policy concerns related to interpretation of probabilistic results in the context of deterministic standards. The consensus of the group was that these issues need to be dealt with carefully and in the overall context of the purpose of performance assessment as a decision making tool. In this context, the general feeling was there should be caution applied to making any rushes to judgment regarding quantitative criteria beyond comparisons of central tendencies of probabilistic results with the deterministic standards. The group agreed there are benefits to reviewing probabilistic approaches used for safety analysis of nuclear facilities for lessons learned and a point of comparison. 
Some caution is needed when interpreting the distribution of results, because of the dependence on the input distributions used, which may reflect simplifications on reality (See discussions in Session 1). Thus, there seemed to be more interest in comparisons with central tendencies (mean or median) for compliance purposes rather than percentiles further out on the distribution (e.g., $95^{\text {th }}$ ). This reflects a desire to use the full distribution of results to inform a decision, but not to set too many numerical criteria for distributions that could potentially force a decision rather than using results other than the central tendencies to inform the decision.

It was recommended to highlight a distinction between assigning probabilities for intruder scenarios and assigning probabilities or some indicator of relative frequency to what-if cases, alternative exposure scenarios, multiple failure configurations, etc. This would be consistent with application of probabilistic approaches used for safety analysis and also with the intent for probabilistic assessments to move more towards realism rather than conservatism. 
WSRC-STI-2008-00333, REVISION 0

\subsection{CONCLUSIONS AND RECOMMENDATIONS}

Following the policy discussion, a brief summary discussion was used as an opportunity to summarize some of the main impressions from the workshop and also to identify a set of key recommendations as conclusions from the workshop. Overall, the workshop was successful in meeting the objectives that were set:

- Review applications of probabilistic performance assessments and share technical perspectives, lessons learned and challenges to implementation,

- Provide a forum for practitioners to identify good practices and share experiences,

- Improve consistency in application of probabilistic assessments,

- Address key implementation and policy issues, and

- Identify references for information that can help improve the application of probabilistic approaches.

In the opening remarks for the Workshop, it was pointed out that it had been several years since such a gathering of people involved in performance assessments had been held. In the context of improving consistency, the importance of fostering improved communication among the people conducting assessments was recognized by all and this workshop was believed to be an effective means of fostering communication.

\subsection{GENERAL IMPRESSIONS}

The presentations and discussions highlighted the fact that there remains healthy skepticism regarding both probabilistic and deterministic approaches. The group did not see this as a negative. The consensus was this skepticism simply reflects the need for PA practitioners to demonstrate that a thoughtful approach has been used for the assessment rather than relying on a technique to do the thinking. The importance of conducting PAs that are focused on the specific objectives (generally, informing a decision regarding compliance) rather than treating the effort as a modeling exercise was also emphasized many times. PA models can be so complex, it is essential to keep such an objective in mind throughout the process to avoid getting sidetracked on activities that do not contribute to achieving the real goal.

The importance of an iterative approach using sensitivity analysis as a tool was highlighted throughout the discussions and presentations. Although people tend to think of uncertainty analysis first, it was apparent that the sensitivity analysis aspect of the work was a critical consideration for the PA process. The role of sensitivity analysis in identifying future work that has the best potential to benefit the PA and also to guide reviewers to key aspects of the analysis was consistently highlighted. The need for the sensitivity analysis (and the PA in general) to be focused on the decision to be made was highlighted numerous times as well. These considerations have resulted in the term "importance analysis" being used to emphasize that it is not simply global model sensitivity, but identifying sensitive parameters specific to the decision to be made. Approaches for implementation of the sensitivity analysis part of the PA were clearly an area of interest. 
A few key technical/policy areas were the subject of much discussion. Concerns about how input distributions are being developed were apparent. Several examples were discussed that illustrated how problems with the definition of distributions have resulted in strange output for the PA models that were resolved through further refinement of the input distributions.. There was a lot of discussion about how to know when it is necessary to use a probabilistic approach. There is some expense and time associated with developing a probabilistic model and the inputs needed, so there needs to be a recognized benefit. The general feeling was that it is difficult to set specific criteria, a priori. The consensus seemed to be more along the lines that it is important to recognize the points where the benefits outweigh the costs. Along those lines, it was discussed that there can be many secondary benefits of developing a simplified model amenable to a probabilistic approach, beyond simply enabling the probabilistic calculations. The need to highlight these benefits was identified.

One of the important challenges in adopting a probabilistic approach is developing the model that is amenable to the multiple realizations required for a probabilistic approach. One session was devoted to this topic in recognition of the challenges. Significant progress has been made in abstracting the details from a three-dimensional model into a more simplified representation amenable to multiple realizations needed for a full probabilistic assessment.

\subsection{OVERALL RECOMMENDATIONS}

The workshop concluded with six key recommendations based on the presentations and discussions that took place:

1. There was a desire for guidance on how to determine what level of complexity is needed and when there is a need to implement a probabilistic approach. It is not clear whether an a priori determination for these issues is generally possible. The general view was to maintain proper perspective that the PA process is intended to inform decisions and deterministic and probabilistic approaches should be used with that goal in mind. From this view, decisions regarding when to use deterministic and probabilistic approaches will be based on how much information is needed to make the decision at hand. It was recommended that part of an activity to develop guidance could involve a review of existing positions regarding the use of deterministic and probabilistic methods (e.g., DOE-HS, NRC, EPA, international). A critical aspect of any recommendations will be an emphasis on the importance of conducting sensitivity or "importance" analyses and their use in the iterative assessment approach and the value that can be added by using a "hybrid" approach based on a combination of deterministic and probabilistic calculations. 
2. The most significant technical recommendation was a clear need for additional guidance on the development of input distributions to be used for performance assessments. Experience to date has shown this is one of the most challenging aspects of moving to a probabilistic approach. The uniqueness of the problem for PAs entails a need to make modifications to traditional approaches. It was strongly recommended that a future workshop on this topic would probably be the best single area of focus resulting from this workshop.

3. The group believed it would be very beneficial to create a categorized data repository and relevant references from existing PA efforts that could serve as a resource for practitioners across the complex. Having this centralized resource would reduce time spent searching for data, reduce duplication of effort and also should help promote more consistency in approaches. The task should also be linked with similar activities for other organizations (e.g., ISCORS). A retrospective look at data and references used in existing PAs would be a first step towards building this resource.

4. Model abstraction was another technical issue that poses several challenges. As a first step, the group believed that it would be useful to summarize some of the benefits of having a simplified model available (e.g., ease of conducting special analyses, ease of explanation to stakeholders, QA of detailed model, composite analysis). This discussion could be included with a summary of some fundamental criteria to be considered when trying to develop a simplified representation of a more complex model.

5. There is a need for specific guidance on addressing uncertainties beyond variability (conceptual models, alternative exposure scenarios, multiple failure scenarios, etc.). There is a need for the ability to consider probabilities for some of these situations, especially in cases of highly unlikely what-if scenarios.

6. Two general topics were highlighted that need to be considered for all activities: benefits of involvement of reviewers early and often in the PA process and the need to maintain sufficient QA and documentation for the process, especially in the context of being able to transfer the work to others, if needed. 
WSRC-STI-2008-00333, REVISION 0

\subsection{REFERENCES}

\subsection{SESSION 1}

Cullen, A.C., and H.C. Frey, 1999, Probabilistic Techniques in Exposure AssessmentHandbook for Dealing with Variability and Uncertainty in Models and Inputs, Plenum Press, New York.

Kammen, D. M., and Hassenzzahl, 1999, Should We Risk It? Exploring Environmental, Health, and Technological Problem Solving, Princeton University Press, Princeton, NJ.

Krupnic, A., R. Morgenstern, M. Batz, P. Nelson, D. Burtraw, J-S. Shih, and M. McWilliams, 2006; Not a Sure Thing: Making Regulatory Choices under Uncertainty, Resources for the Future. http://www.rff.org/rff/Documents/RFF-Rpt-RegulatoryChoices.pdf

Morgan M. G., and M. Henrion, 1990, Uncertainty, Cambridge University Press, Cambridge, U.K.

Vose, D., 2000, Risk Analysis - A Quantitative Guide, John Wiley \& Sons, Cambridge, U.K.

\subsection{SESSION 2}

ACRI. 2004. PORFLOW ${ }^{T M}$ Version 5.0 User's Manual, Revision 5, Analytical \& Computational Research, Inc., Los Angeles, California.

GoldSim. 2007a. GoldSim User's Guide. GoldSim Technology Group LLC. Issaquah, Washington.

GoldSim. 2007b. GoldSim Contaminant Transport User's Guide. GoldSim Technology Group LLC. Issaquah, Washington.

Neuman, S.P. and P.J. Wierenga. 2003. A Comprehensive Strategy of Hydrogeologic Modeling and Uncertainty Analysis for Nuclear Facilities and Sites. NUREG/CR-6805. U.S. Nuclear Regulatory Commission. Washington, DC.

Pachepsky, Y.A., A.K. Gruber, M. Th. van Genuchten, T. J. Nicholson, R.E. Cady, J. Šimůnek, and M.G. Schaap. 2006. Model Abstraction Techniques for Soil-Water Flow and Transport. NUREG/CR-6884. U.S. Nuclear Regulatory Commission. Washington, DC. 


\section{APPENDIX A. WORKSHOP AGENDA}

\section{Probabilistic Sensitivity and Uncertainty Analysis Workshop March 10-11, 2008, Augusta, Georgia Augusta Marriott Hotel and Suites, Estes Meeting Room Call-in Number: (803) 725-1403 - Access Code 6858190 (limited lines, please try to call as a group, if possible)}

\begin{tabular}{|c|c|c|}
\hline \multicolumn{3}{|c|}{ Day 1 - March 10 (7:30 a.m. - 5:30 p.m.) } \\
\hline Time & Topic & Presenter \\
\hline 7:30 - 8:00 a.m. & Registration & All \\
\hline 8:00 - 8:30 a.m. & Opening Remarks & $\begin{array}{l}\text { Marty Letourneau } \\
\text { DOE-HQ, EM-11 }\end{array}$ \\
\hline $8: 30$ - 9:00 a.m. & Background for Workshop & $\begin{array}{l}\text { Roger Seitz } \\
\text { SRNL }\end{array}$ \\
\hline 9:00 - 9:05 a.m. & Session 1 - Input Data and Distributions & $\begin{array}{l}\text { Bruce Crowe } \\
\text { NTS - Battelle }\end{array}$ \\
\hline 9:05 - 9:35 a.m. & $\begin{array}{l}\text { Probabilistic Analysis and Parameter } \\
\text { Distributions - Experience Learned in } \\
\text { Developing the Probabilistic RESRAD }\end{array}$ & $\begin{array}{c}\text { Charley Yu } \\
\text { ANL }\end{array}$ \\
\hline 9:35 - 10:05 a.m. & $\begin{array}{c}\text { Development of Input Data and } \\
\text { Distributions for Performance } \\
\text { Assessment }\end{array}$ & $\begin{array}{l}\text { David Esh } \\
\text { U.S. NRC }\end{array}$ \\
\hline $\begin{array}{l}\text { 10:05-10:30 } \\
\text { a.m. }\end{array}$ & Break & \\
\hline $\begin{array}{l}\text { 10:30-11:00 } \\
\text { a.m. }\end{array}$ & $\begin{array}{l}\text { How to Construct Defensible Input } \\
\text { Distributions }\end{array}$ & $\begin{array}{c}\text { Paul Black } \\
\text { Neptune and Co. }\end{array}$ \\
\hline $\begin{array}{c}\text { 11:00 - 12:00 } \\
\text { a.m. }\end{array}$ & Moderated Discussion for Session 1 & $\begin{array}{c}\text { Bruce Crowe } \\
\text { NTS - Battelle }\end{array}$ \\
\hline $12: 00-1: 30$ p.m. & Lunch & \\
\hline
\end{tabular}




\begin{tabular}{|c|c|c|}
\hline \multicolumn{3}{|c|}{ Day 1- March 10 (7:30 a.m. - 5:30 p.m.) - continued } \\
\hline Time & Topic & Presenter \\
\hline 1:30-1:35 p.m. & Session 2 - Model Abstraction & $\begin{array}{c}\text { Mike Sully } \\
\text { Neptune and Co. }\end{array}$ \\
\hline 1:35-2:05 p.m. & $\begin{array}{c}\text { Model Abstraction for the F Tank Farm } \\
\text { Performance Assessment }\end{array}$ & $\begin{array}{c}\text { Greg Flach/Glenn Taylor } \\
\text { SRNL }\end{array}$ \\
\hline 2:05-2:35 p.m. & $\begin{array}{c}\text { Groundwater Transport Abstraction in a } \\
\text { PA for a LLW facility, Los Alamos, NM }\end{array}$ & $\begin{array}{c}\text { Phil Stauffer } \\
\text { LANL }\end{array}$ \\
\hline 2:35 - 3:00 p.m. & Break & $\begin{array}{c}\text { Matt Kozak } \\
\text { Monitor Scientific }\end{array}$ \\
\hline 3:00-3:30 p.m. & $\begin{array}{c}\text { Model Abstraction and Treatment of } \\
\text { Model Uncertainty }\end{array}$ \\
\hline 3:30-4:30 p.m. & Moderated Discussion for Session 2 & $\begin{array}{c}\text { Mike Sully } \\
\text { Neptune and Co. }\end{array}$ \\
\hline 4:30- 5:30 p.m. & Open Discussion of Sessions 1 and 2 & $\begin{array}{c}\text { Roger Seitz } \\
\text { SRNL }\end{array}$ \\
\hline
\end{tabular}




\begin{tabular}{|c|c|c|}
\hline \multicolumn{3}{|c|}{ Day 2 - March 11 (8:30 a.m. - 5:30 p.m.) } \\
\hline Time & Topic & Presenter/ Organization \\
\hline $8: 30-9: 00$ a.m. & Summary from Sessions 1 and 2 & Bruce Crowe/Mike Sully \\
\hline 9:00 - 9:05 a.m. & $\begin{array}{c}\text { Session } 3 \text { - Implementation of Probabilistic } \\
\text { Sensitivity and Uncertainty Analyses }\end{array}$ & $\begin{array}{c}\text { Marc Wood } \\
\text { Fluor Hanford }\end{array}$ \\
\hline $9: 05-9: 35$ a.m. & $\begin{array}{c}\text { Sensitivity Analysis for Low-Level Waste } \\
\text { Performance Assessment }\end{array}$ & $\begin{array}{l}\text { Art Rood } \\
\text { INL }\end{array}$ \\
\hline $9: 35$ - 10:05 a.m. & $\begin{array}{c}\text { So That All Works in Theory - How Does } \\
\text { It Happen? Implementation Issues in } \\
\text { Uncertainty/Sensitivity Analysis }\end{array}$ & $\begin{array}{c}\text { John Tauxe } \\
\text { Neptune and Co. }\end{array}$ \\
\hline 10:05 - 10:30 a.m. & Break & \\
\hline $10: 30-11: 00$ a.m. & $\begin{array}{c}\text { The Roles of Uncertainty Analysis and } \\
\text { Sensitivity Analysis in Real World Decision } \\
\text { Making }\end{array}$ & $\begin{array}{c}\text { Paul Black } \\
\text { Neptune and Co. }\end{array}$ \\
\hline $11: 00-12: 00$ a.m. & Moderated Discussion for Session 3 & $\begin{array}{c}\text { Marc Wood } \\
\text { Fluor Hanford }\end{array}$ \\
\hline $12: 00-1: 30$ p.m. & Lunch & \\
\hline $1: 30-2: 00$ p.m. & Summary of Session 3 & Marc Wood \\
\hline $2: 00-2: 05$ p.m. & Session 4 - Policy Implications & $\begin{array}{l}\text { Roger Seitz } \\
\text { SRNL }\end{array}$ \\
\hline $2: 05-2: 35$ p.m. & EM-11 Perspectives & $\begin{array}{l}\text { Marty Letourneau } \\
\text { DOE-HQ, EM-11 }\end{array}$ \\
\hline $2: 35-3: 05$ p.m. & HS-20 Perspectives & $\begin{array}{c}\text { Andy Wallo } \\
\text { DOE-HQ, HS-20 }\end{array}$ \\
\hline 3:05 - 3:30 p.m. & Break & \\
\hline $3: 30-4: 30$ p.m. & Moderated Discussion for Session 4 & $\begin{array}{l}\text { Roger Seitz } \\
\text { SRNL }\end{array}$ \\
\hline $4: 30-5: 30$ p.m. & Path Forward - Open Discussion & $\begin{array}{l}\text { Marty Letourneau } \\
\text { Roger Seitz }\end{array}$ \\
\hline
\end{tabular}


APPENDIX B. LIST OF PARTICIPANTS

\begin{tabular}{|c|c|c|c|c|}
\hline NAME & COMPANY & $\begin{array}{c}\text { SITE } \\
\text { LOCATION } \\
\end{array}$ & $\begin{array}{c}\text { TELEPHONE } \\
\text { NUMBER } \\
\end{array}$ & E-MAIL ADDRESS \\
\hline Angwafo, Maatsi & DOE & SRS/HQ & $803-208-1156$ & maatsi.angwafo@em.doe.gov \\
\hline Bentley, Jeffrey & DOE & SRS/HQ & $803-648-1269$ & jeffrey.bentley@em.doe.gov \\
\hline Bonczek, Rich & $\mathrm{DOE}$ & PPPO & $859-219-4051$ & rich.bonczek@lex.doe.gov \\
\hline Black, Paul & Neptune \& Co. & Various & $720-746-1803$ & pblack@neptuneinc.org \\
\hline Blocker, Rosalind & SRNL & SRS & $803-725-7745$ & rosalind.blocker@srnl.doe.gov \\
\hline Burns, Heather & SRNL & SRS & $706-863-5920$ & heather.burns@srnl.doe.gov \\
\hline Butcher, Tom & SRNL & SRS & $803-725-5810$ & tom.butcher@srnl.doe.gov \\
\hline Carter, Anna & DOE & ID & $208-526-6410$ & carterac@id.doe.gov \\
\hline Collard, Len & SRNL & SRS & $803-613-0372$ & leonard.collard@srnl.doe.gov \\
\hline Connelly, Michael & $\mathrm{CH} 2 \mathrm{M}$ & Hanford & 509-373-3891 & michael_connelly@rl.gov \\
\hline Crowe, Bruce & Battelle & $\mathrm{NV}$ & $702-295-0519$ & bruce.crowe@nv.doe.gov \\
\hline Dickert, Virginia & WSRC & SRS & $803-952-8700$ & virginia.dickert@srs.gov \\
\hline Dimenna, Richard & SRNL & SRS & $803-725-8203$ & richard.dimenna@srnl.doe.gov \\
\hline Esh, David & US NRC & Headquarters & $301-415-6705$ & david.esh@nrc.gov \\
\hline Flach, Greg & SRNL & SRS & $803-725-5195$ & gregory.flach@srnl.doe.gov \\
\hline Frantz, Aaron & $\mathrm{CDM}$ & Portsmouth & $610-293-0450$ & frantzar@cdm.com \\
\hline French, Sean & LANS & LANL & $505-667-5953$ & sbf@lanl.gov \\
\hline Goldston, Sonny & WSRC & SRS & $803-507-1310$ & sonny.goldston@srs.gov \\
\hline Greeves, John & PEC & $\mathrm{HQ}$ & $301-452-3511$ & greevesj@aol.com \\
\hline Hiergesell, Bob & SRNL & SRS & $803-725-5219$ & robert.hiergesell@srnl.doe.gov \\
\hline Johnstone, Fraser & PRS & Paducah & $270-441-5206$ & edward.johnstone@prs-llc.net \\
\hline Knepp, Tony & YAHSGS & Hanford & $509-438-6453$ & anthony_j_knepp@rl.gov \\
\hline Kozak, Matt & Monitor Sci. & Denver & $303-985-0005$ & mkozak@monitorsci.com \\
\hline Krenzien, Susan & Navarro & $\mathrm{NV}$ & $702-295-1618$ & krenziens@nv.doe.gov \\
\hline Langton, Chris & SRNL & SRS & $803-725-5806$ & christine.langton@srnl.doe.gov \\
\hline Layton, Mark & WSRC & SRS & $803-208-0450$ & mark.layton@srs.gov \\
\hline Letourneau, Martin & DOE & HQ & $301-903-3532$ & martin.letourneau@em.doe.gov \\
\hline
\end{tabular}


WSRC-STI-2008-00333, REVISION 0

\begin{tabular}{|c|c|c|c|c|}
\hline NAME & COMPANY & $\begin{array}{c}\text { SITE } \\
\text { LOCATION } \\
\end{array}$ & $\begin{array}{c}\text { TELEPHONE } \\
\text { NUMBER }\end{array}$ & E-MAIL ADDRESS \\
\hline Mahadevan, Sankaran & Vanderbilt & Various & $615-322-3040$ & sankaran.mahadevan@vanderbilt.edu \\
\hline McCarthy, James & CWI & Idaho Falls & $208-526-8319$ & james.mccarthy@icp.doe.gov \\
\hline Meyer, Philip & PNNL & Hanford & $503-417-7552$ & philip.meyer@pnl.gov \\
\hline Newman, Jeff & WSRC & SRS & $803-206-3215$ & jeffry.newman@srs.gov \\
\hline Patterson, John & Strata-G & Paducah & $865-934-3403$ & jpatterson@stratag.org \\
\hline Pinkston, Karen & US NRC & $\mathrm{HQ}$ & $301-415-3650$ & karen.pinkston@nrc.gov \\
\hline Pope, Howard & DOE & SRS & $803-208-6218$ & howard.pope@srs.gov \\
\hline Rafferty, Melda & $\mathrm{DOE} / \mathrm{PPPO}$ & Portsmouth & $740-897-5521$ & melda.rafferty@lex.doe.gov \\
\hline Regnier, Edward & DOE & HQ & $202-586-5027$ & edward.regnier@eh.doe.gov \\
\hline Reid, Luke & WSRC & SRS & $803-557-6309$ & luke.reid@srs.gov \\
\hline Rood, Art & INL & Idaho Falls & $208-526-1678$ & arthur.rood@inl.gov \\
\hline Robinson, Tom & WSRC & SRS & $803-208-3443$ & tom.robinson@srs.gov \\
\hline Rosenberger, Kent & WSRC & SRS & $803-208-3147$ & kent.rosenberger@srs.gov \\
\hline Ross, Sherri & $\mathrm{DOE}$ & SR & $803-208-6078$ & sherri.ross@srs.gov \\
\hline Seitz, Jolene & $\mathrm{DOE}$ & SRS & $803-208-6234$ & jolene.seitz@srs.gov \\
\hline Seitz, Roger & SRNL & SRS & $803-725-8269$ & roger.seitz@srnl.doe.gov \\
\hline Sharp, Dave & LATA/Parallax & Portsmouth & $740-897-4169$ & dsharp@lpports.com \\
\hline Shott, Greg & NSTEC & NTS & $702-295-7388$ & shottgj@nv.doe.gov \\
\hline Shuman, Rob & URS Corp & LANL & $207-639-2269$ & rshuman@gwi.net \\
\hline Simmons, Mike & DOE-SR & SRS & $803-208-6328$ & jonathan.simmons@srs.gov \\
\hline Smith, Danny & Mentat & $\mathrm{HQ}$ & $703-505-7845$ & danny_smith_rpe@msn.com \\
\hline Smith, Frank & SRNL & SRS & $803-725-9780$ & frank02.smith@srnl.doe.gov \\
\hline Stanisich, Nick & Portage & Idaho Falls & 406-683-2836 & nstanisich@portageenv.com \\
\hline Stauffer, Phil & LANL & Los Alamos & $505-665-4638$ & stauffer@lanl.gov \\
\hline Sully, Michael & New Mexico & Los Alamos & $505-827-6187$ & michael.sully@state.nm.us \\
\hline Swingle, Rob & SRNL & SRS & $803-725-2369$ & rob.swingle@srnl.doe.gov \\
\hline Snook, Jeff & $\mathrm{DOE}$ & Paducah & $270-441-6814$ & jeff.snook@lex.doe.gov \\
\hline Tauxe, John & Neptune & Various & $505-662-2121$ & jtauxe@neptuneinc.org \\
\hline Taylor, Glenn & SRNL & SRS & $803-725-5823$ & glenn.taylor@srnl.doe.gov \\
\hline Thomas, Steve & WSRC & SRS & $803-208-8064$ & steven.thomas@srs.gov \\
\hline
\end{tabular}


WSRC-STI-2008-00333, REVISION 0

\begin{tabular}{|l|l|l|l|l|}
\hline \multicolumn{1}{|c|}{ NAME } & \multicolumn{1}{|c|}{ COMPANY } & \multicolumn{1}{c|}{$\begin{array}{c}\text { SITE } \\
\text { LOCATION }\end{array}$} & $\begin{array}{c}\text { TELEPHONE } \\
\text { NUMBER }\end{array}$ & \multicolumn{1}{|c|}{ E-MAIL ADDRESS } \\
\hline Thorne, David & Portage & Idaho Falls & $970-243-5237$ & dthorne@portageenv.com \\
\hline Wallo, Andy & DOE & HQ & $202-586-4996$ & andrew.wallo@hq.doe.gov \\
\hline Wilhite, Elmer L. & SRNL & SRS & $803-725-5800$ & elmer.wilhite@srnl.doe.gov \\
\hline Willcox, Mary V. & DOE & ID & 208-526-2173 & willcomv@id.doe.gov \\
\hline Wood, Marc & Fluor & Hanford & $509-373-3308$ & marcus_i_wood@rl.gov \\
\hline Yu, Charley & ANL & Argonne & $630-252-5589$ & cyu@anl.gov \\
\hline Yucel, Vefa & NSTEC & NTS & $702-295-7388$ & yucelv@nv.doe.gov \\
\hline
\end{tabular}


WSRC-STI-2008-00333, REVISION 0

This page intentionally left blank. 
WSRC-STI-2008-00333, REVISION 0

APPENDIX C. PRESENTATIONS

INTRODUCTION 
WSRC-STI-2008-00333, REVISION 0

This page intentionally left blank. 

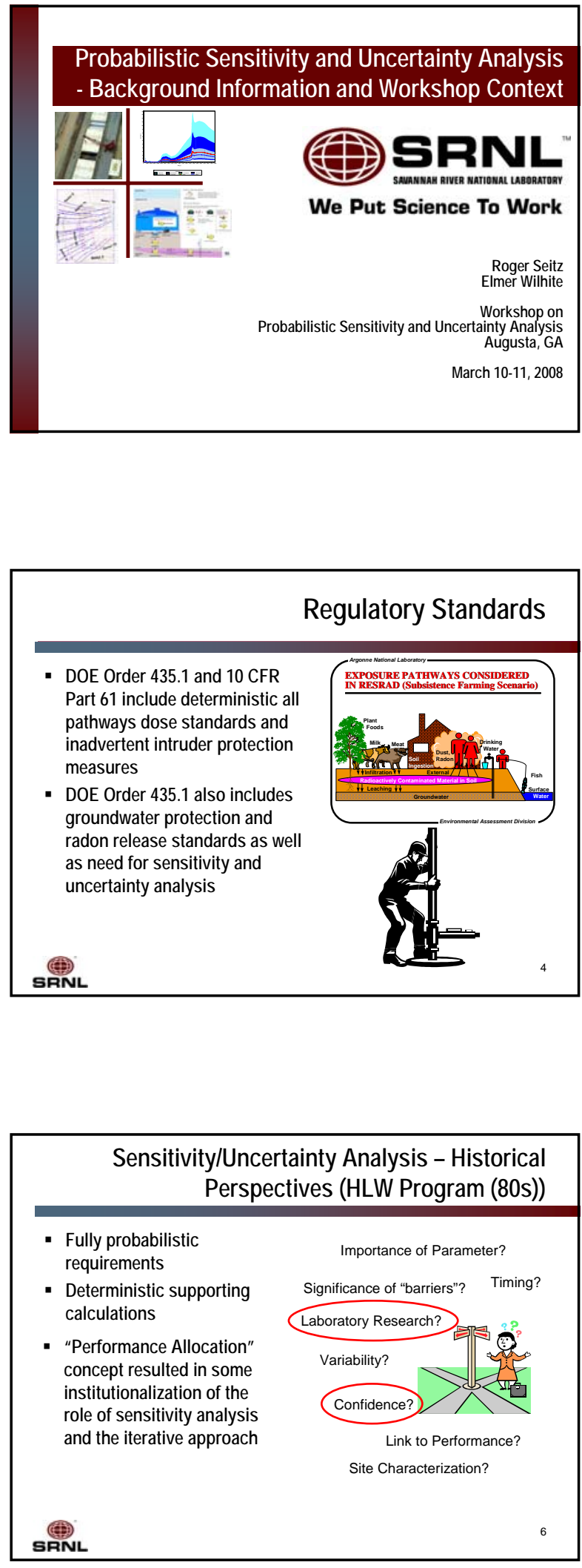
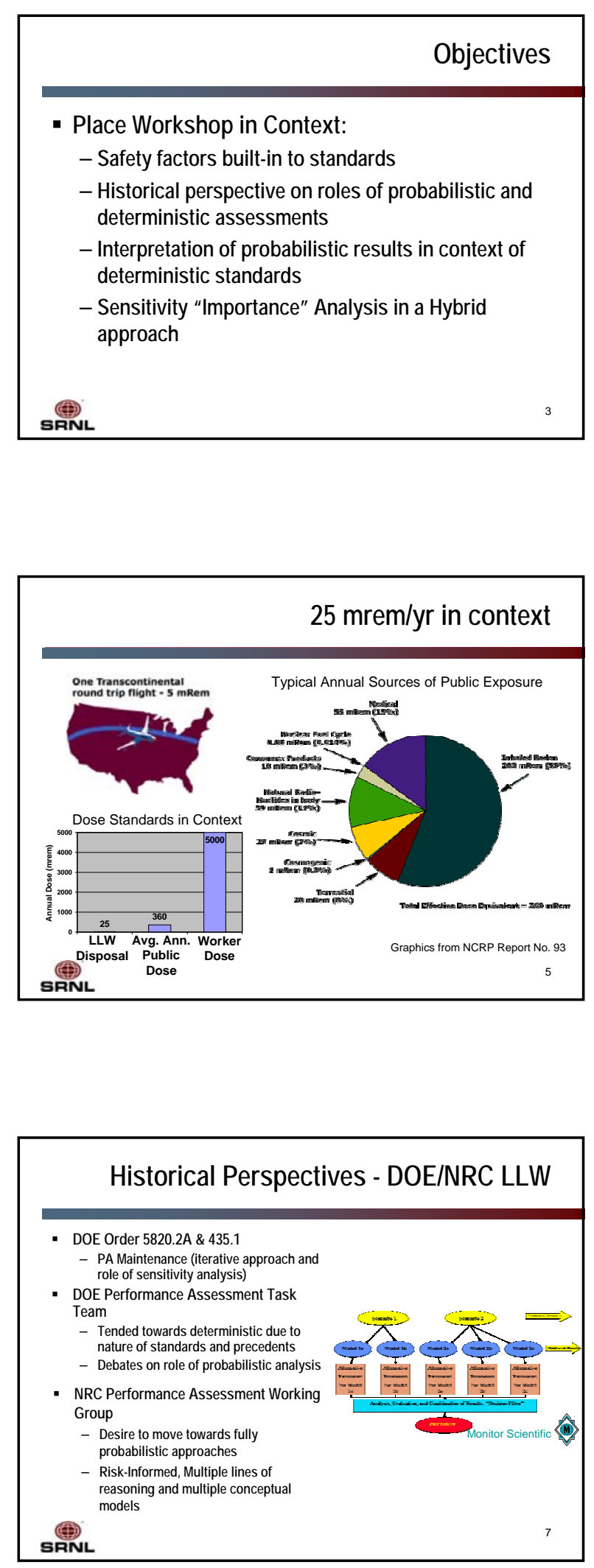

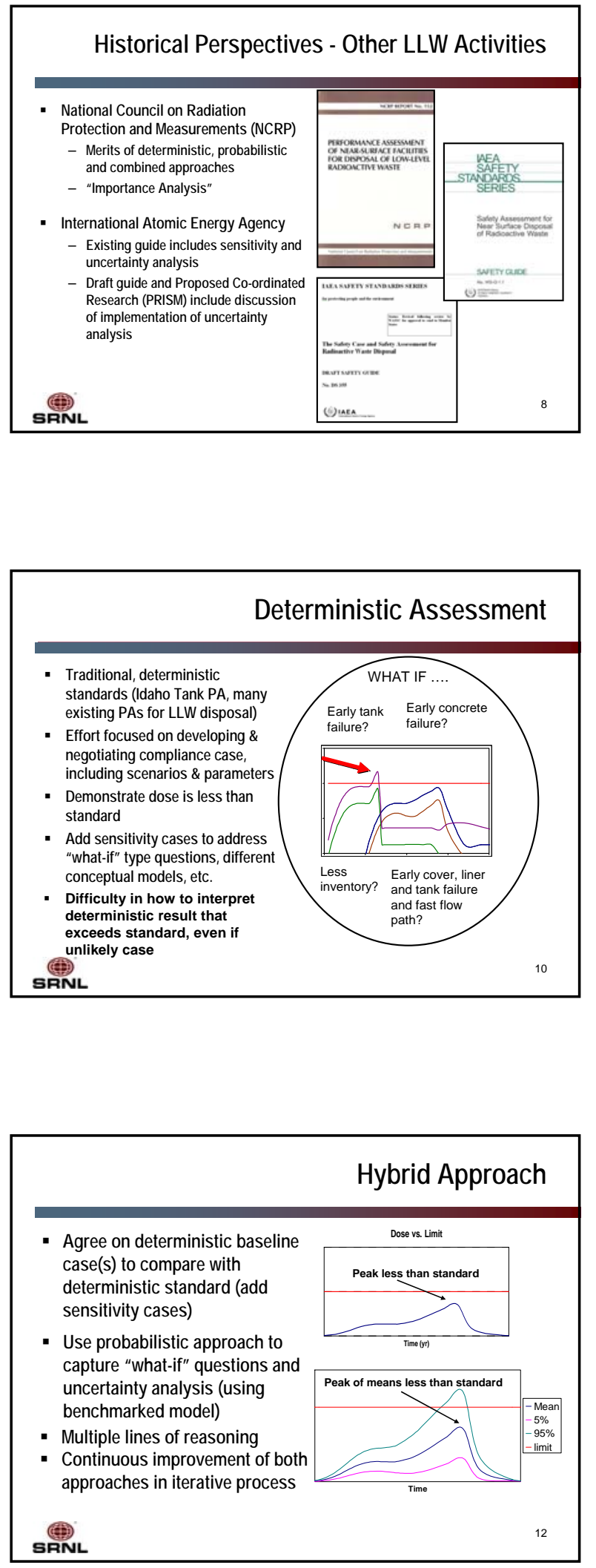

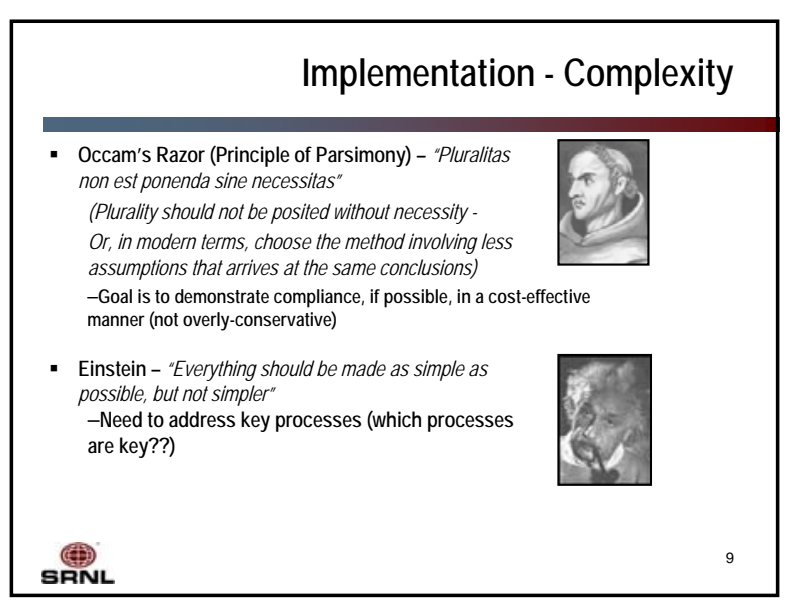

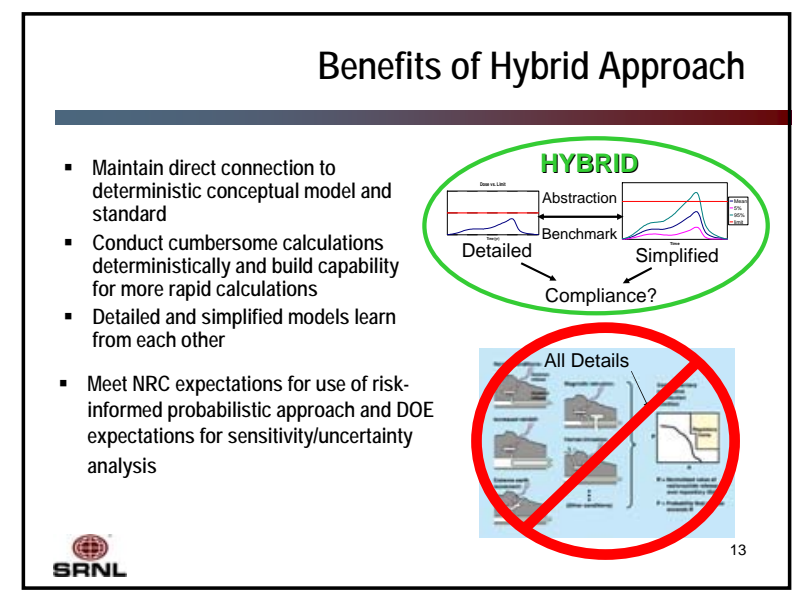



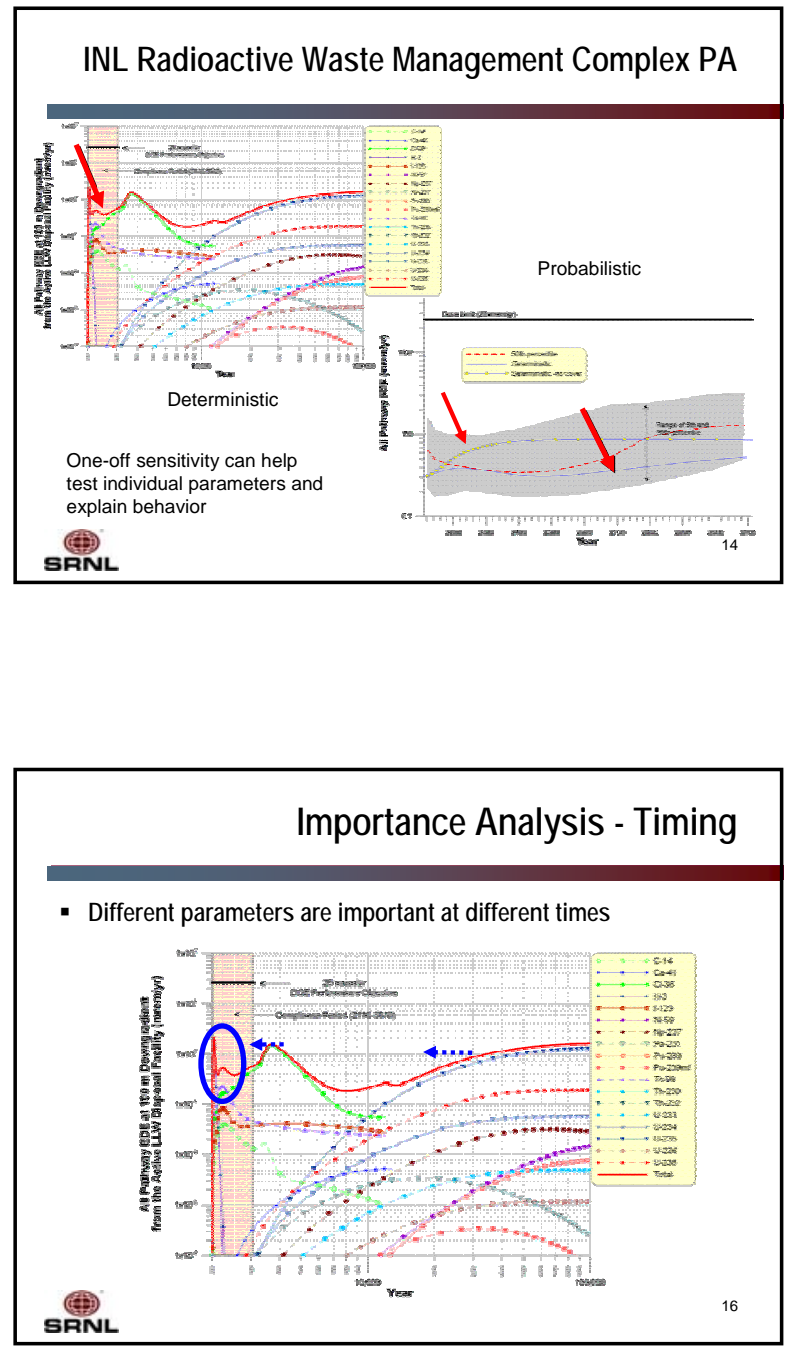

\section{Summary}

- Deterministic regulations include safety factors

- Value of sensitivity and uncertainty analysis as part of PA has been recognized for many years

- Deterministic approaches have been successful for many cases, but addressing "what-ifs" can be problematic

- Probabilistic approaches provide a means to implicitly consider uncertainty and not consider extreme cases individually

- Sensitivity (Importance) analyses need to be interpreted carefully

- Don't get consumed by implementing methods, no approach is a substitute for thinking and demonstrating system understanding

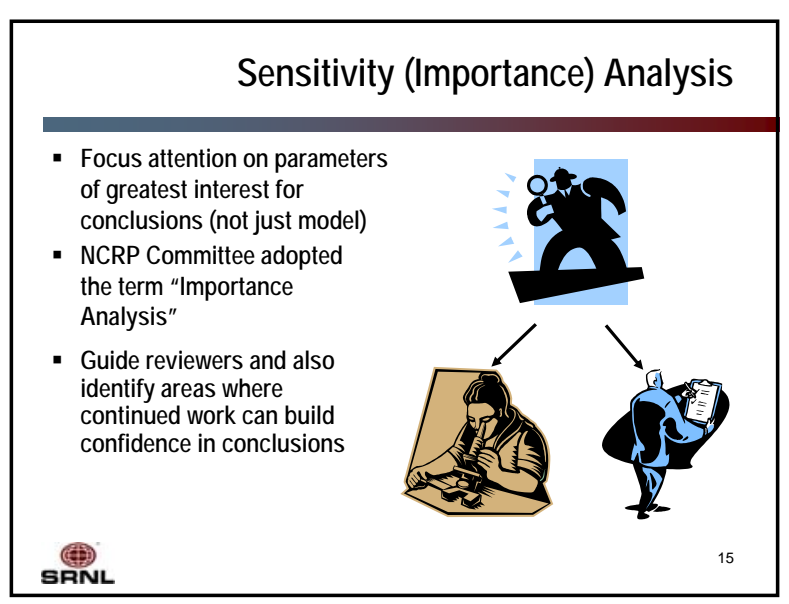

\begin{tabular}{|c|c|c|c|c|c|c|}
\hline \multicolumn{7}{|c|}{ Importance Analysis - Probabilistic } \\
\hline \multicolumn{7}{|c|}{ INL RWMC Example - Timing } \\
\hline \multirow{2}{*}{$\begin{array}{l}500 \text { years after } \\
\text { Disposal }\end{array}$} & \multirow{2}{*}{$\begin{array}{c}\text { Rank } \\
\text { Correlation } \\
\text { Coefficient } \\
\end{array}$} & \multirow{2}{*}{$\begin{array}{c}\text { Percent } \\
\text { Variance } \\
(\%)\end{array}$} & \multicolumn{4}{|c|}{ SRS Tank Farm Example - Endpoint } \\
\hline & & & \multirow[t]{2}{*}{ First 10,000 years } & \multicolumn{3}{|c|}{ Sensitivity Index } \\
\hline \begin{tabular}{|c|}
$\mathrm{Cl}-36$ source term \\
\end{tabular} & & 37 & & Well A & Well B & All Wells \\
\hline & -0.27 & 27 & Tank X failure scenario & 11 & - & 3.7 \\
\hline longevity (year) & & & Vadose zone thickness & 5.6 & 6.8 & 3.1 \\
\hline \multirow[t]{2}{*}{ Uranium Kd (mL/g) } & -0.23 & 19 & Pu Kd (sandy soil) & 4.9 & 11 & 5.5 \\
\hline & & & Saturated aquifer thickness & 4.4 & 6.4 & 7.3 \\
\hline 2000 years after & Rank & Percent & \begin{tabular}{|l} 
Pu Kd (clayey soil) \\
\end{tabular} & - & 4.9 & $\cdot$ \\
\hline Disposal & $\begin{array}{l}\text { Correlation } \\
\text { Coefficient }\end{array}$ & $\begin{array}{c}\text { Variance } \\
(\%)\end{array}$ & \multirow{3}{*}{\multicolumn{4}{|c|}{$\begin{array}{l}\text { Iterative approach to identify key } \\
\text { contributors, then focus sensitivity analysis }\end{array}$}} \\
\hline Uranium $\mathrm{Kd}(\mathrm{mL} / \mathrm{g})$ & -0.5 & 67 & & & & \\
\hline Cl-36 source term & 0.2 & 11 & & & & \\
\hline \multicolumn{3}{|l|}{ SRNL } & & & & 17 \\
\hline
\end{tabular}

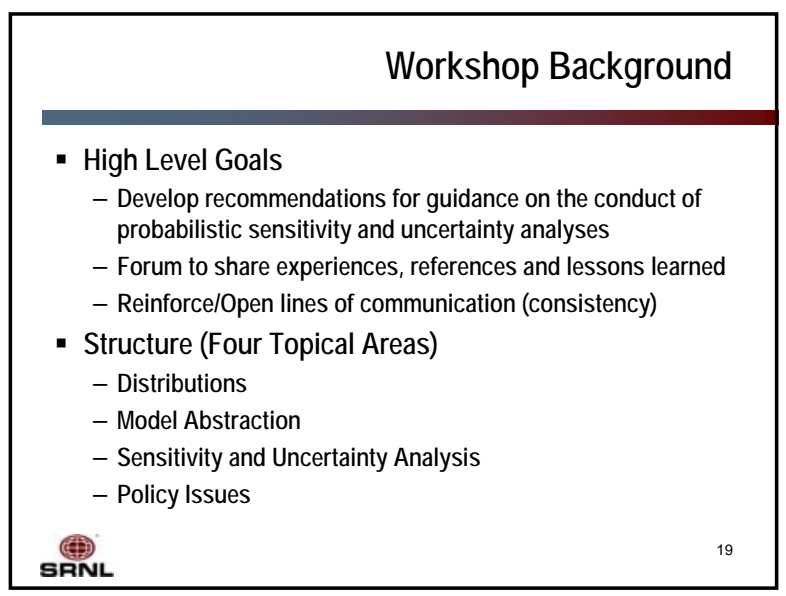




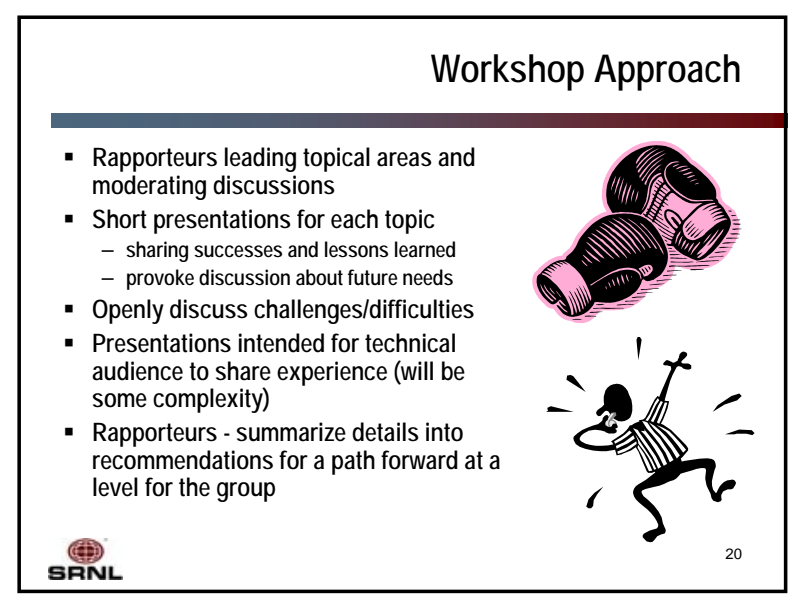

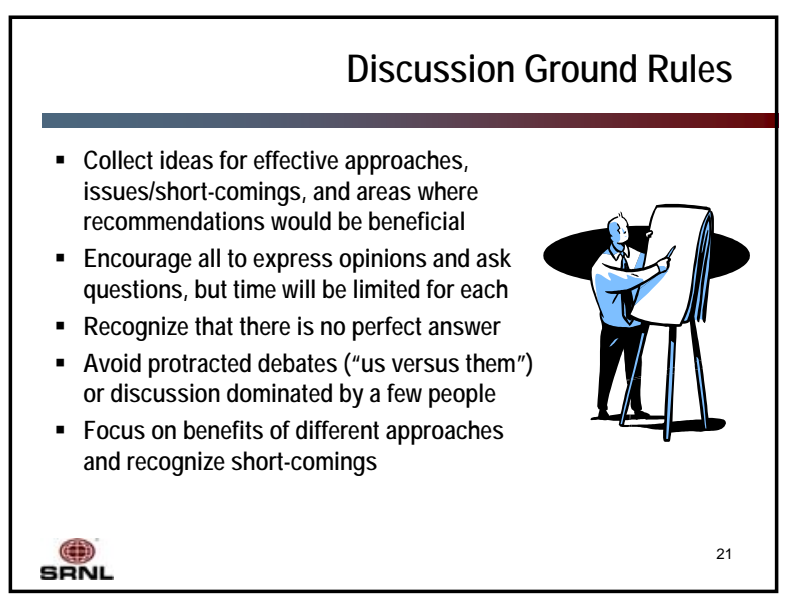


WSRC-STI-2008-00333, REVISION 0

\section{SESSION 1 - INPUT DATA AND DISTRIBUTIONS}


WSRC-STI-2008-00333, REVISION 0

This page intentionally left blank. 

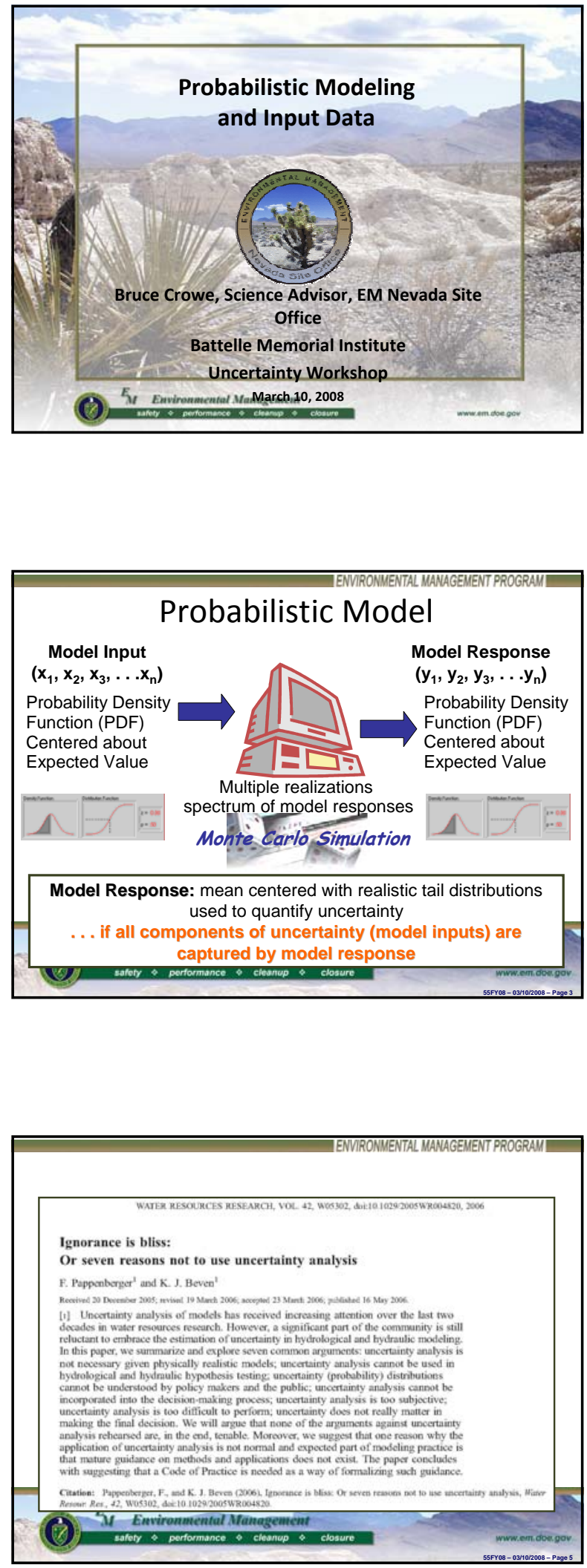
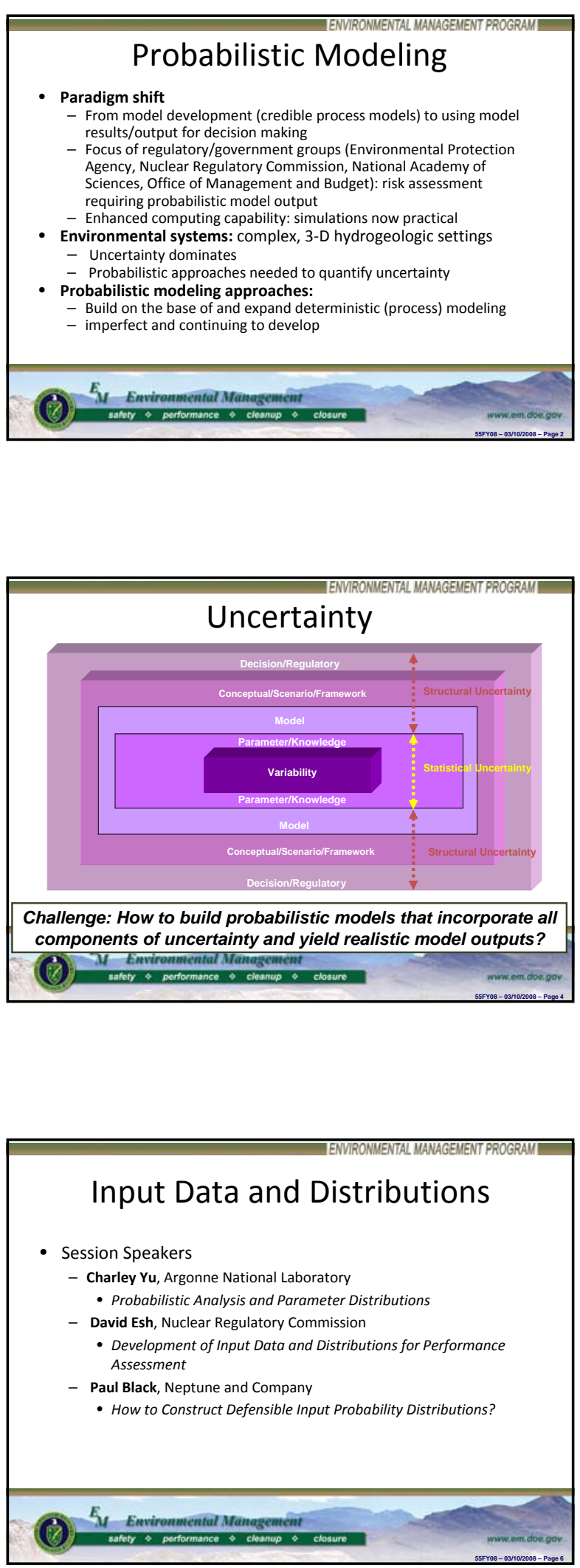

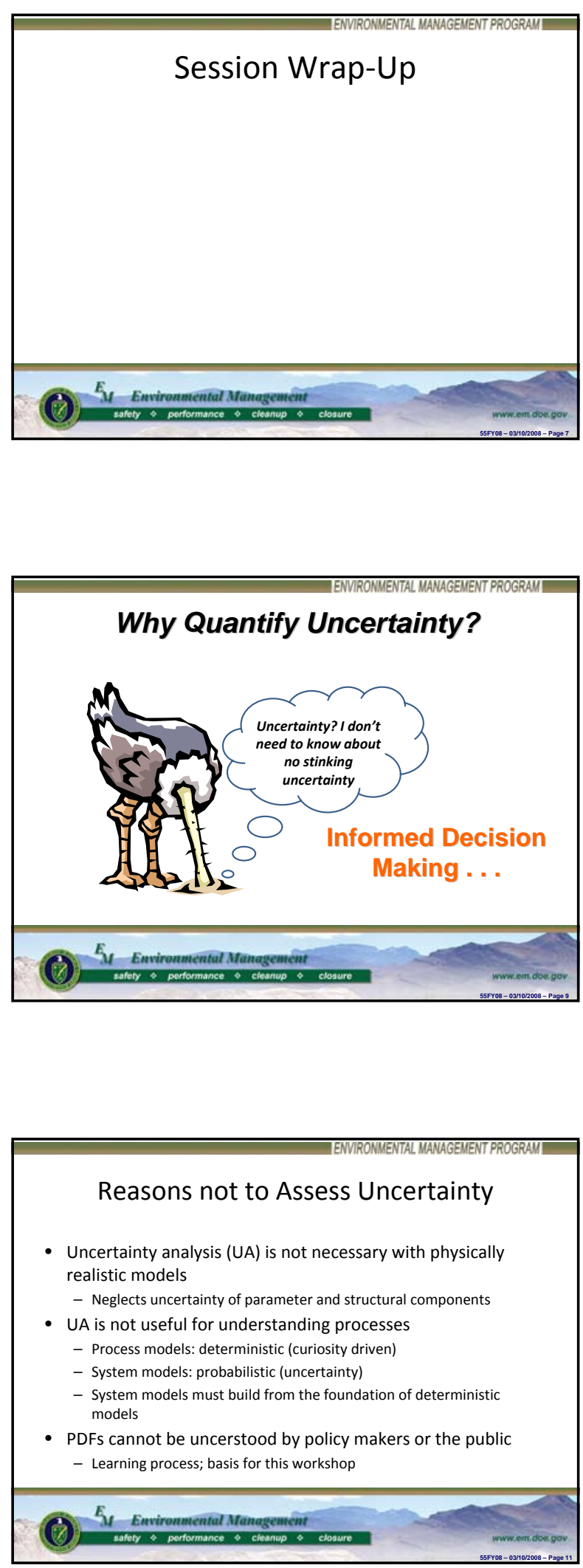
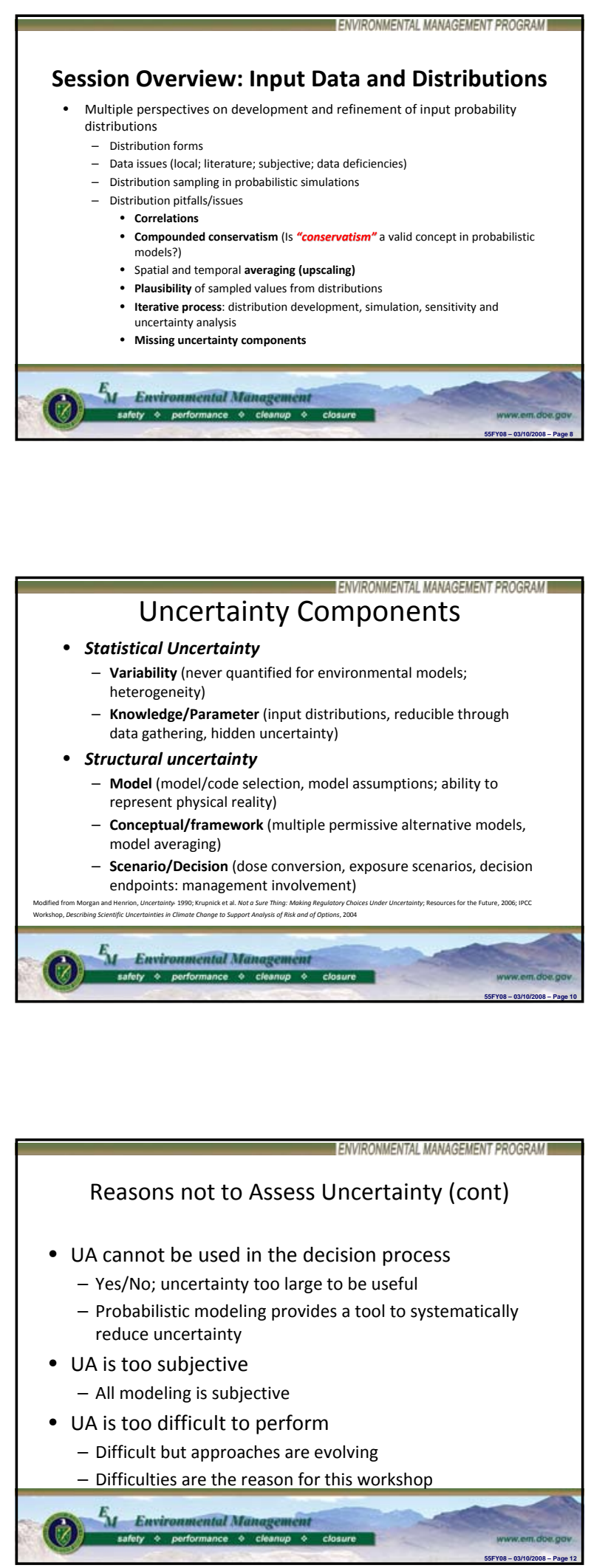


\begin{tabular}{|c|c|}
\hline Argonne & $\begin{array}{l}\text { Probabilistic Analysis and } \\
\text { Parameter Distributions } \\
\text { Experience Learned in Developing } \\
\text { the Probabilistic RESRAD Codes }\end{array}$ \\
\hline & $\begin{array}{l}\text { Charley Yu, PhD, CHP } \\
\text { RESRAD Program Manager } \\
\text { Presented at the Probabilistic Sensitivity and } \\
\text { Uncertainty Analysis Workshop }\end{array}$ \\
\hline $\begin{array}{l}\text { (3) } \\
\text { UChicagor } \\
\text { Argone. }\end{array}$ & $\begin{array}{l}\text { March 10-11, } 2008 \\
\text { Augusta, Georgia }\end{array}$ \\
\hline
\end{tabular}

\section{Schematic Overview of Probabilistic Analysis}

Probabilistic Analysis

Monte Carlo or LHS Sampling
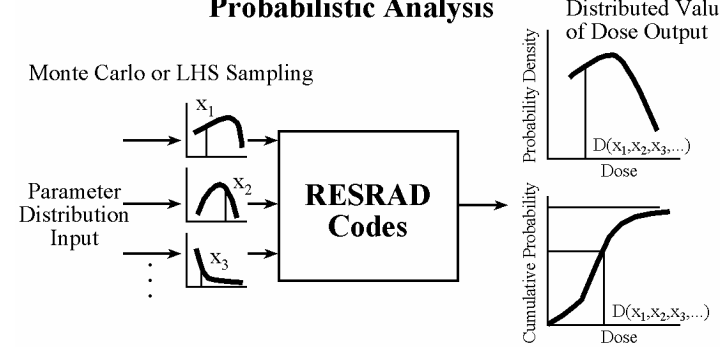

Argonne 4

Use of Dose Results
\begin{tabular}{|l|l|}
\hline \multicolumn{1}{|c|}{ Deterministic } & \multicolumn{1}{c|}{ Probabilistic } \\
\hline Based on the peak dose & $\begin{array}{l}\text { Based on some measure of the } \\
\text { distribution of the peak dose } \\
\text {-A percentilee (e.g., 99\%, 95\%) of } \\
\text { the distribution of the peak dose, } \\
\text {-The mean of the peak doses, } \\
\text {-The peak of a percentile of the } \\
\text { distribution of the dose over time, } \\
\text {-The peak of the mean doses over } \\
\text { time }\end{array}$ \\
\hline $\begin{array}{l}\text { Consistently used for various types } \\
\text { of dose assessment }\end{array}$ & $\begin{array}{l}\text { Not consistent? } \\
\text { Need guidance? }\end{array}$ \\
\hline \multicolumn{2}{|c|}{ Environmental science Division } \\
\hline
\end{tabular}

\section{Presentation Outline}

What is Probabilistic Analysis

- The difference between deterministic analysis and probabilistic analysis

- Compilation of Parameter Distributions

- Precautions on using default distributions

- Sampling the Distributions

- Monte Carlo and Latin hypercube sampling

- Grouping the Samples

- Correlated or random

- Discussion

- How certain are you that you are doing it right?

\section{Argonne 4}

Comparison of Deterministic and Probabilistic Analysis

\begin{tabular}{|l|l|}
\hline \multicolumn{1}{|c|}{ Deterministic } & \multicolumn{1}{c|}{ Probabilistic } \\
\hline $\begin{array}{l}\text { Single value for each input } \\
\text { parameter }\end{array}$ & $\begin{array}{l}\text { A distribution for one or more } \\
\text { parameters }\end{array}$ \\
\hline Use one set of inputs & $\begin{array}{l}\text { Sample the distribution many } \\
\left(\mathrm{N}_{\text {obs }}\right) \text { times and use } \mathrm{N}_{\text {obs }} \text { sets of } \\
\text { inputs, then repeat } \mathrm{N}_{\text {rep }} \text { times }\end{array}$ \\
\hline $\begin{array}{l}\text { Calculations are performed one } \\
\text { time }\end{array}$ & $\begin{array}{l}\text { Calculations are performed } \\
\text { many }\left(\mathrm{N}_{\text {obs }} \times \mathrm{N}_{\text {rep }}\right) \text { times }\end{array}$ \\
\hline $\begin{array}{l}\text { One result } \\
\text { the peak dose } \\
\text { •the temporal plot of dose }\end{array}$ & $\begin{array}{l}\text { Many sets of results } \\
\text { - a distribution of } \\
\text { •the peak dose } \\
\text {-the temporal plot of dose }\end{array}$ \\
\hline
\end{tabular}

Argonne 4

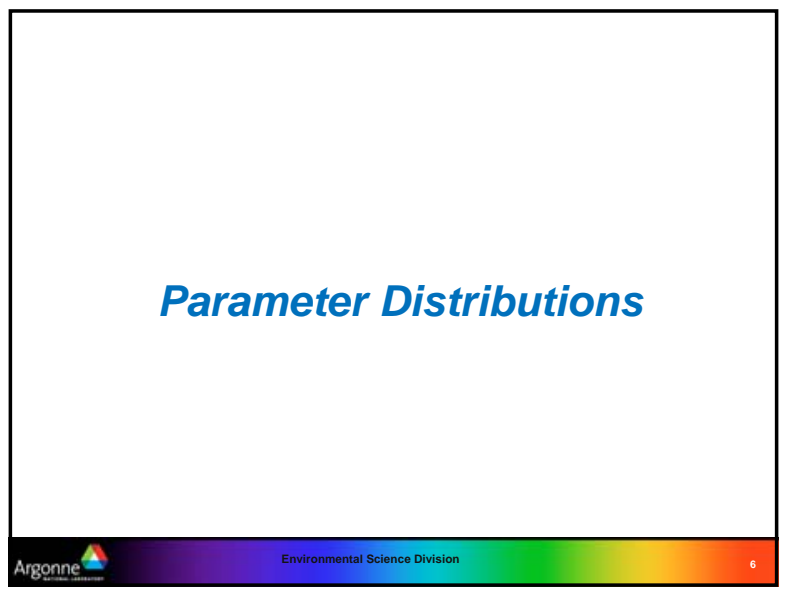




\section{Compilation of Distributions for Input Parameters}

- National statistical distributions are available for many physical parameters

- Most behavioral and metabolic parameter distributions are based on adult male receptors

- Distributions dependent on data amount - distribution fit if sufficient data - restricted by known values otherwise

- Can choose from 19 statistical distributions when specifying site specific distributions

- There are more than 30 ways to specify distributions in RESRADOFFSITE code

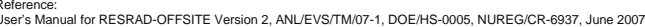

\section{Argonne 4}

Unsaturated Zone Thickness Distribution

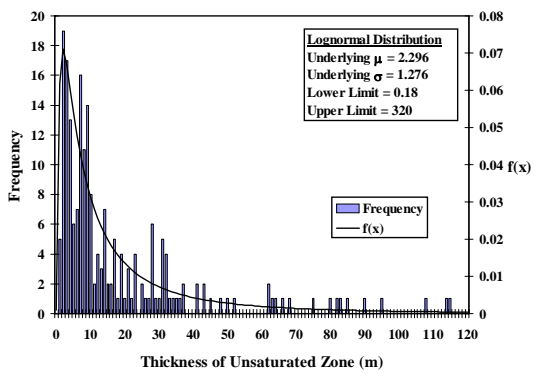

Argonne 4

Precautions on Using Default Distributions

- National (and Regional) Average Distributions

- Unsaturated zone thickness

- Precipitaion, evapotranspiration rate, etc.

- Time Average Distributions

- Mass Loading Factor

- Can short-term dust loading be used for long-term dose assessment (annual dose, dose at 10,000 years, etc.)?

- Precipitation Rate

- Hourly vs. annual precipitation

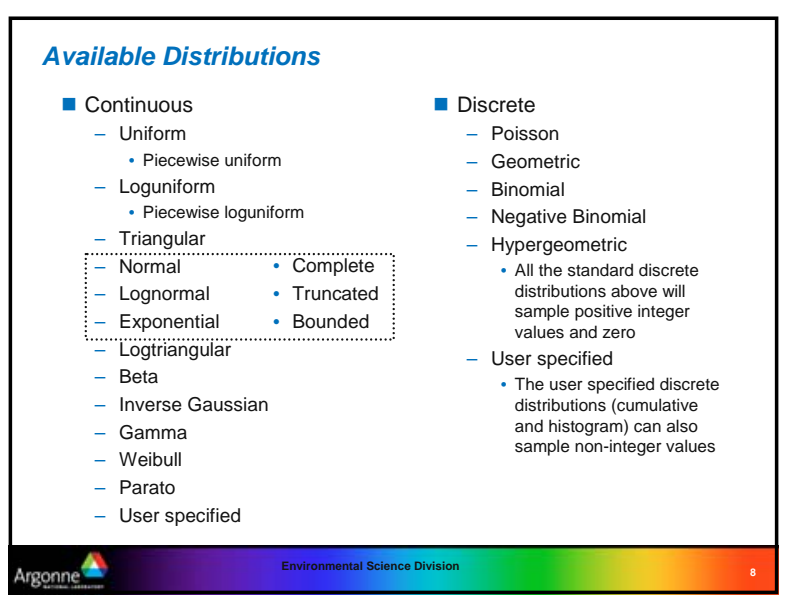

Mass Loading Distribution

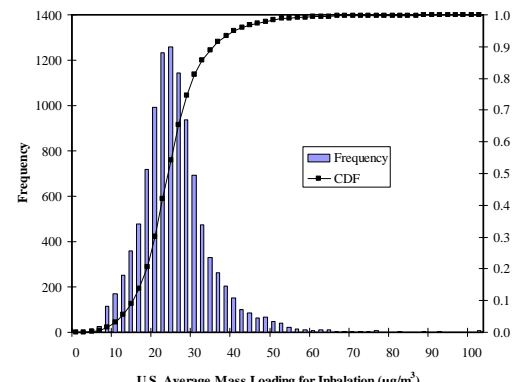

U.S. Average Mass Loading for Inhalation $\left(\mu \mathrm{g} / \mathrm{m}^{3}\right)$

Using Empirical Distributions for Site-specific Data

- Site-specific data can be used directly without having to fit it to a common distribution

- Continuous Distribution Options

- Piecewise Uniform (specify number of observations)

- Continuous Linear (specify cdf)

- Continuous Frequency (specify frequency) 


\section{An Example of Continuous Empirical Distributions}

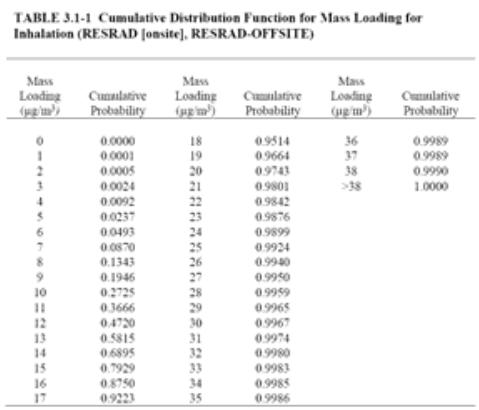

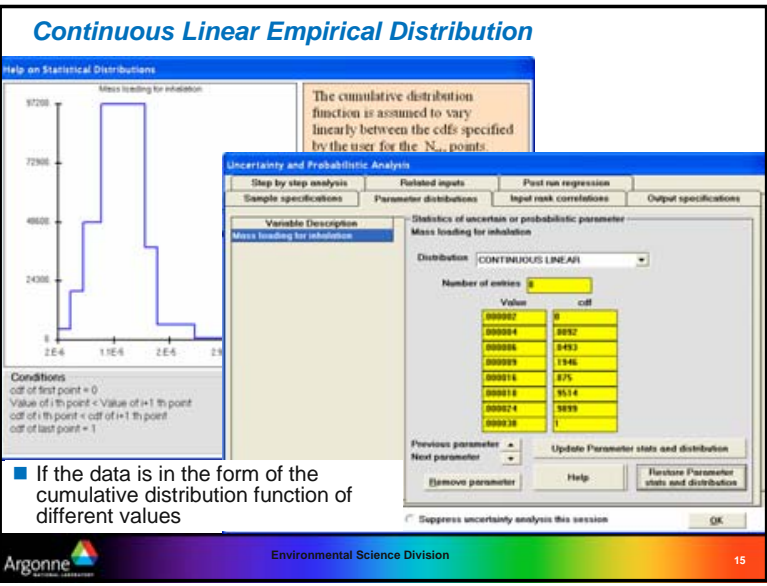

Implementing a Probabilistic Input

- Parameter distributions can not be used directly

- Parameter distributions are "sampled" to reflect the

- Many sampling routines have been developed - Simple Random Sampling

- SRS

- Monte Carlo

- Latin Hypercube Sampling

- LHS specified distributions

\section{An Example of Continuous Empirical Distributions (CDF)}

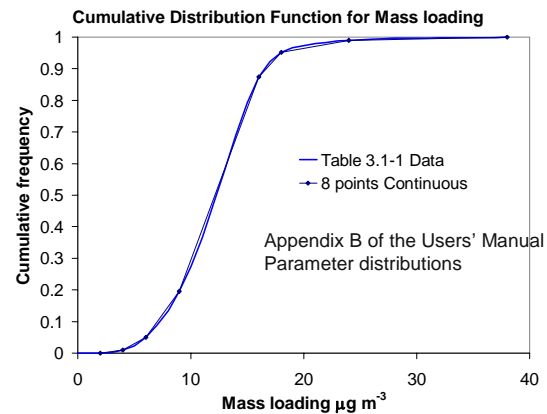

Argonne $\Delta$

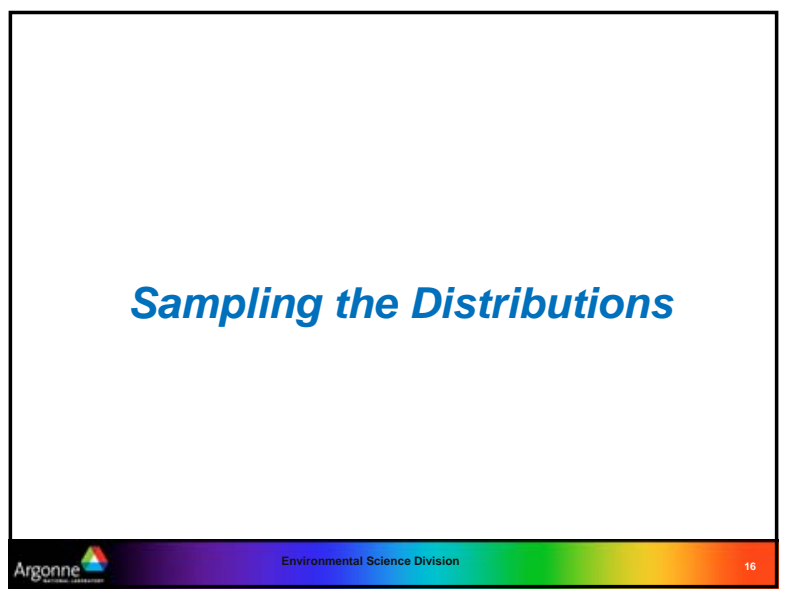

\section{Sampling Methods}

- Simple random sampling "Monte Carlo"

- The entire parameter distribution is sampled randomly based on the number of observations ( $\mathrm{N}_{\mathrm{obs}}$ ) requested by the user

- Latin Hypercube Sampling "LHS"

- Divides the parameter distribution into $\mathrm{N}_{\mathrm{obs}}$ nonoverlapping areas of equal probability

- One sample is obtained from each region 


\section{Combining the Samples}

Grouping of Observations (Highs with Lows)

now should the observations be paired?

- "Highs" with the "lows",

"Lows" with the "highs"?

- Different from highest with lowest, next highest with next lowest, etc.

- Need some way to specify how close you want the paired values to be.

- $\underline{\text { Correlation coefficient }}$

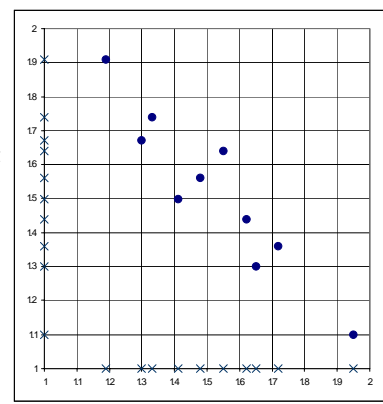

ne

\section{Grouping of Observations (Random)}

- How should the

observations be

paired?

- At "random"?

- May be correlated by

chance

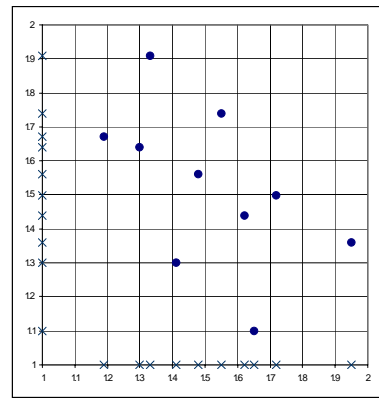

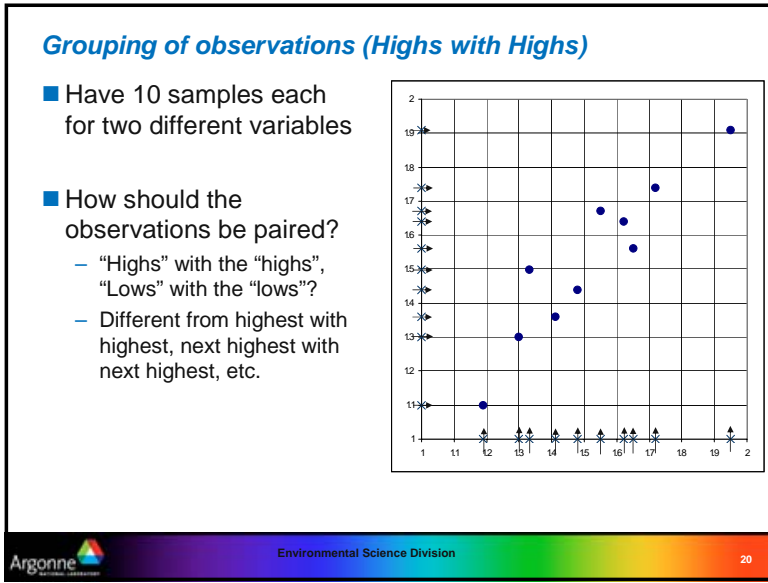

Grouping of Observations (No Correlation)

- How should the

observations be

paired?

- With no correlation?

- Not the same as at

random

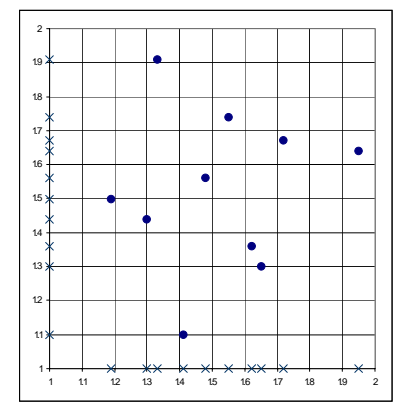

Argonne

Why Correlate Input Parameters?

- There may be a practical reason for a correlation between the input parameters

- Precipitation rate and irrigation rate. More precipitation there is during the (Negative correlation) growing season, less is the need for irrigatio Outdoor time fraction and drinking The amount of water consumed may water intake rate. (Positive

Indoor time fraction and outdoor time Can not add up to be more than 1 fraction

- There may be a theoretical relationship between input parameters

- Porosity and dry bulk density of soil Bulk density $=$ Particle density $\times$ (1- porosity The distribution coefficient of different Depends mainly on the element, minor isotopes, e.g. $238 \mathrm{U}, 234 \mathrm{U}, 235 \mathrm{U}$ isotopic differences due energy of recoil

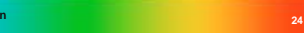




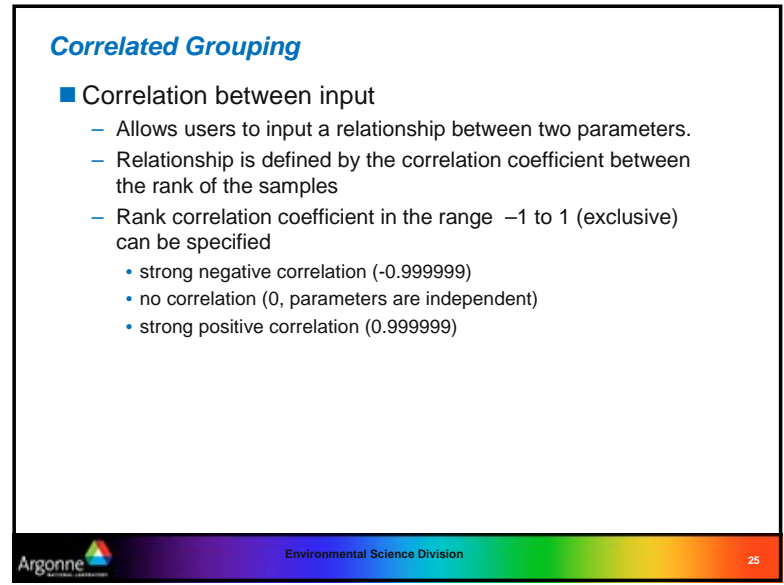

\section{Specifying Correlations Among a Group of Inputs}

- The correlations specified need to be complete and compatible

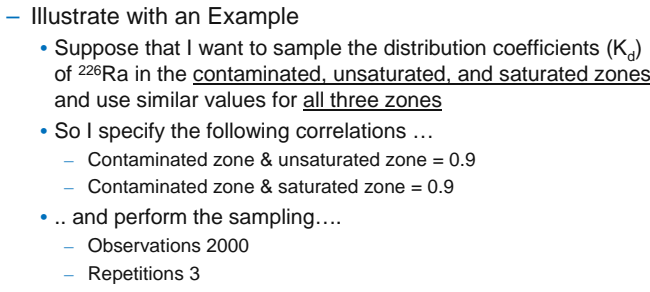

- Suppose that I want to sample the distribution coefficients $\left(K_{d}\right)$ of ${ }^{226} \mathrm{Ra}$ in the contaminated, unsaturated, and saturated zones and use similar values for all three zones

- So I specify the following correlations ...

Contaminated zone \& unsaturated zone $=0.9$

Contaminated zone \& saturated zone $=0.9$

-.. and perform the sampling...

Observations 2000

Repetitions 3

-What will the code do? Will I get what I want to accomplish?

Discussion

- How can one be certain that the code is doing what you think it is doing?

Do I always need site-specific data distributions?

- When can I use default distributions?

- Parameter sensitivity and uncertainty?

- How many observations? How many repetitions?

Which dose value to use? Peak of the means, 95\%ile, or Mean of the peaks? Others?

- Will training help?

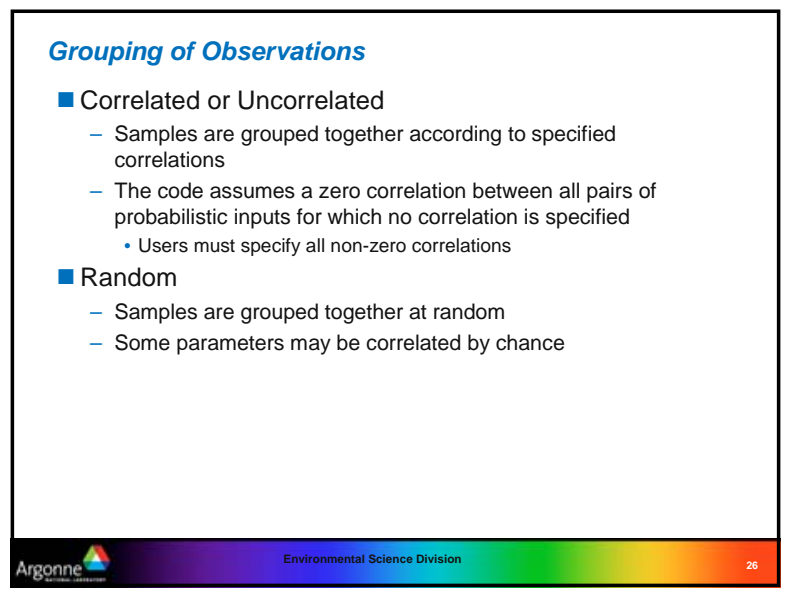

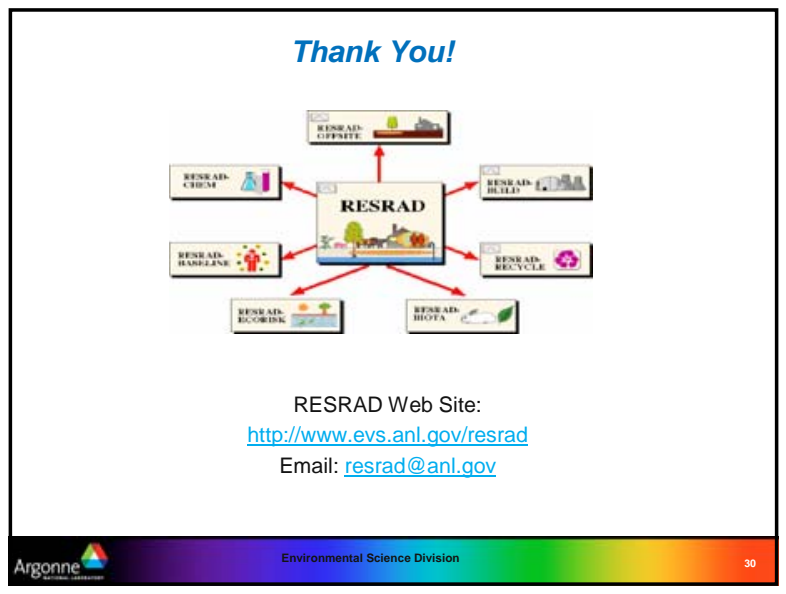




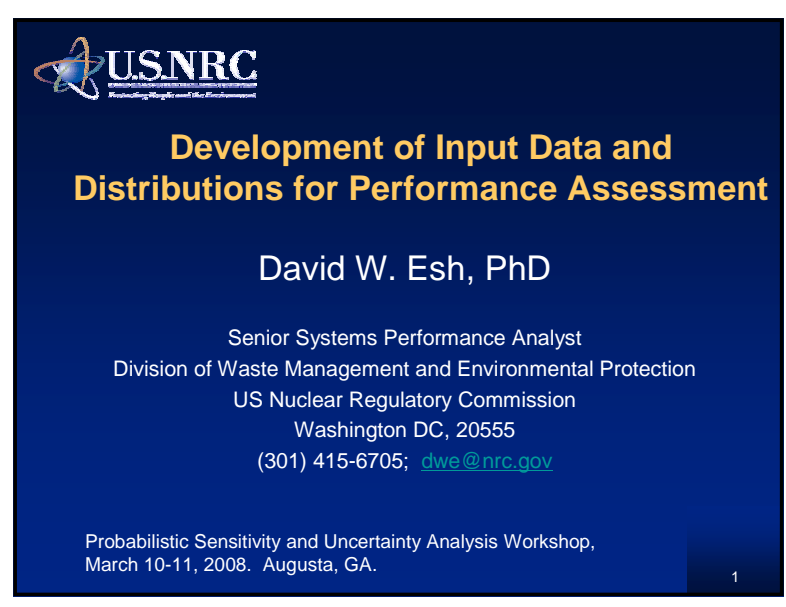

\section{की USNRC}

\section{Data Sources}

- Data for performance assessment - hard to find

- Reasonable sources of data are some of the PA's for specific sites (e.g., NTS, SRS, Hanford)

- Generic datasets available from some regulatory reports

- Wikipedia approach for PA data?
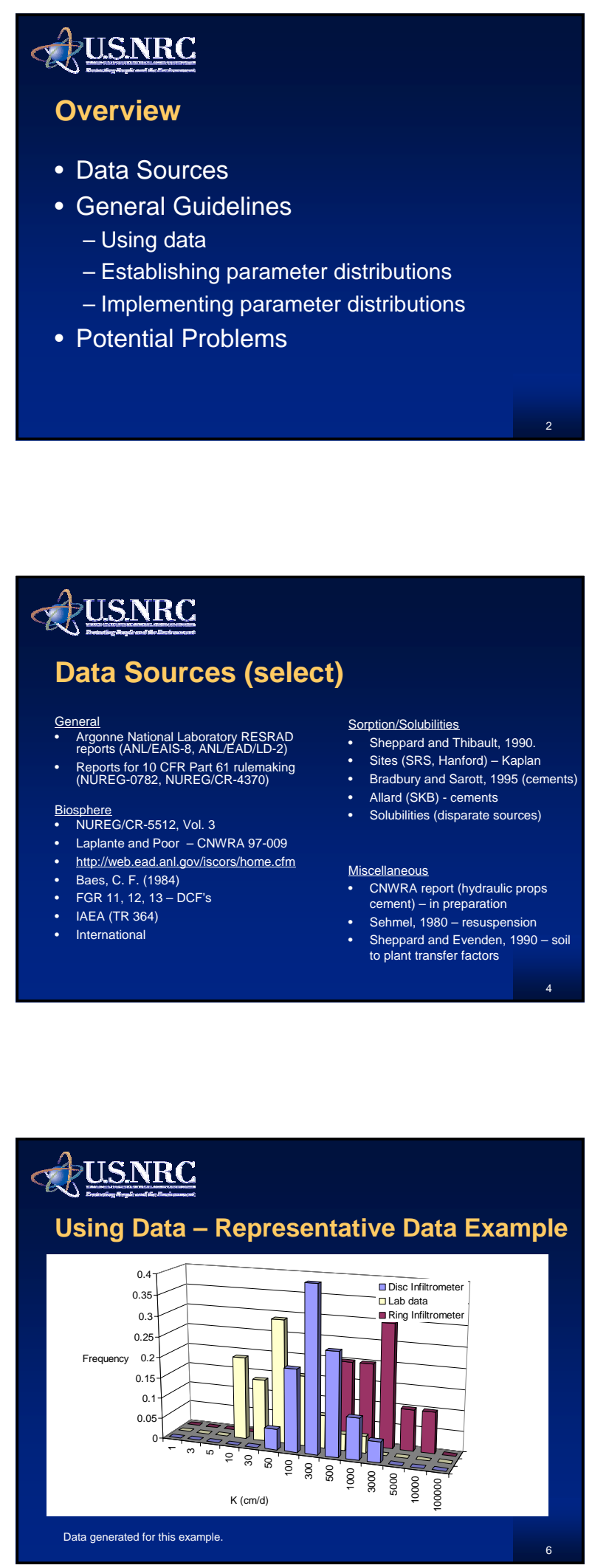

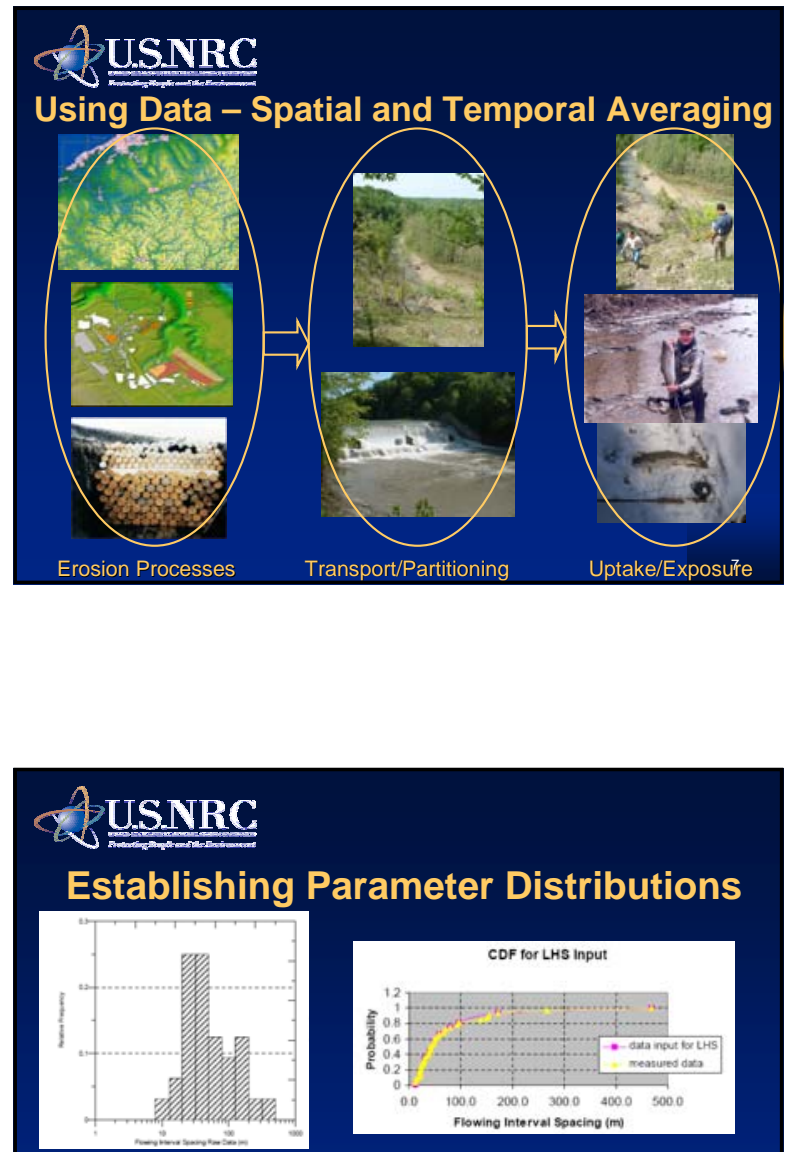

ANL-NBS-MD-000003 Rev 01 September 2004 - Probability Distribution for Flowing Interval Spacing

\section{क्रे USNRC}

\section{Establishing Parameter Distributions}

- A lot of data - use statistical methods

- Limited data:

- Initially use maximum entropy approaches

- Refine distributions by collection of more data

- Truncate distributions that impact timing of dose impacts using conservative bias

- Show comparison of data to distribution

\section{Q U.SNRC}

Implementing Parameter Distributions

- Random sampling - Monte Carlo

- Importance sampling

- Latin Hypercube Sampling (LHS)

- Others

McKay, M.D., R.J. Beckman, and W.J. Conover. 1979. "A Comparison of Three Methods for Selecting Values of Input Variables in the Analysis of Output from a Computer Code," Technometrics. Vol. 21, no. 2, pp. 239-245.

J. C. Helton, "Mathematical and Numerical Approaches in Performance Assessment for Radioactive Waste Disposal: Dealing with Uncertainty," in Modelling Radioactivity in the Environment, E. M. Scott, Ed. New York: Elsevier Science, 2003, pp. 353-390.

\section{\& USNRC}

\section{Problems}

- Unit conversions

- Example: Dry weight or wet weight plant transfer factors

- Lack of correlation

- Example: Food and plant intake rates in probabilistic simulations

- Data replacing a model

- Example: Distribution coefficients (Kd's) from generic sources

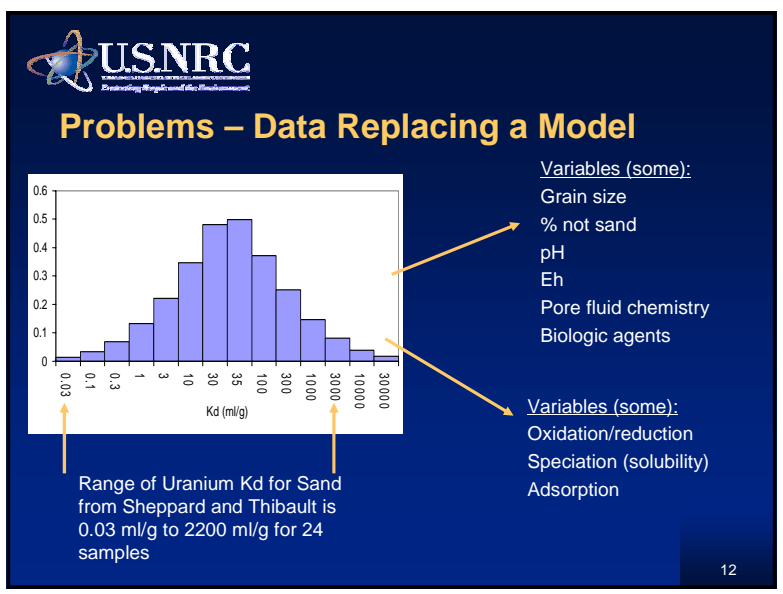




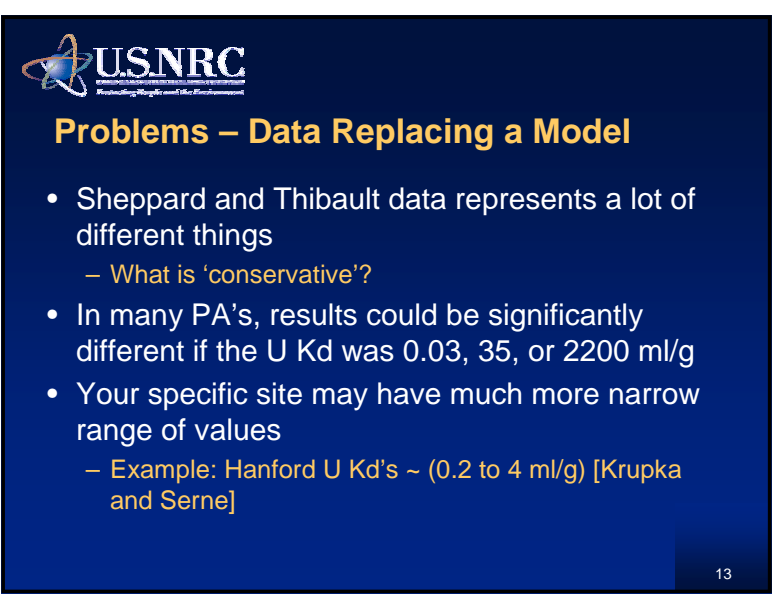

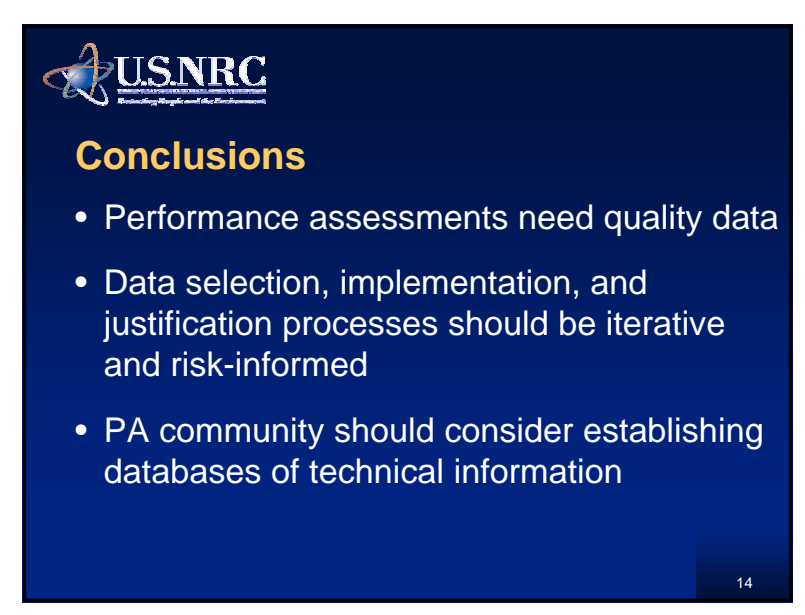




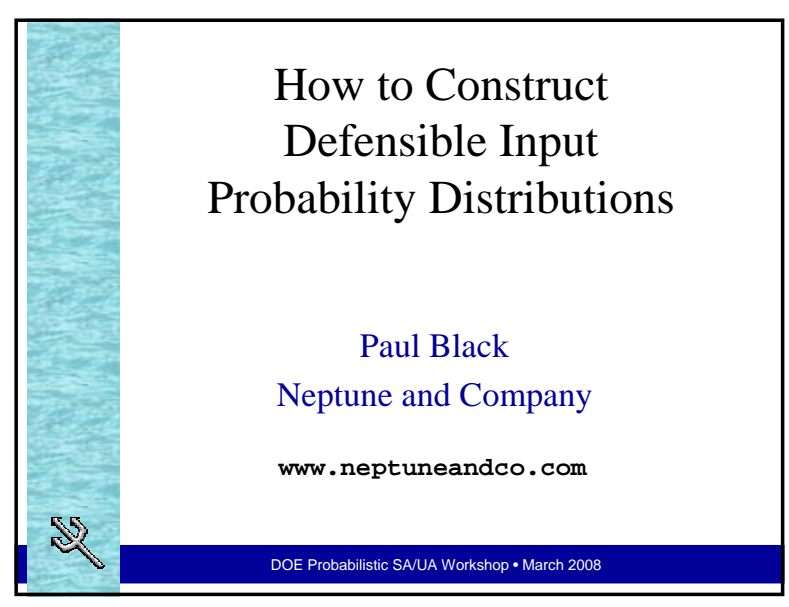

\subsection{Decision Context}

- Compliance assessment

- Comparison to performance objectives

- PA Maintenance

- Reduce uncertainty

- Test conceptual models

- Increase confidence in PA results

- ALARA

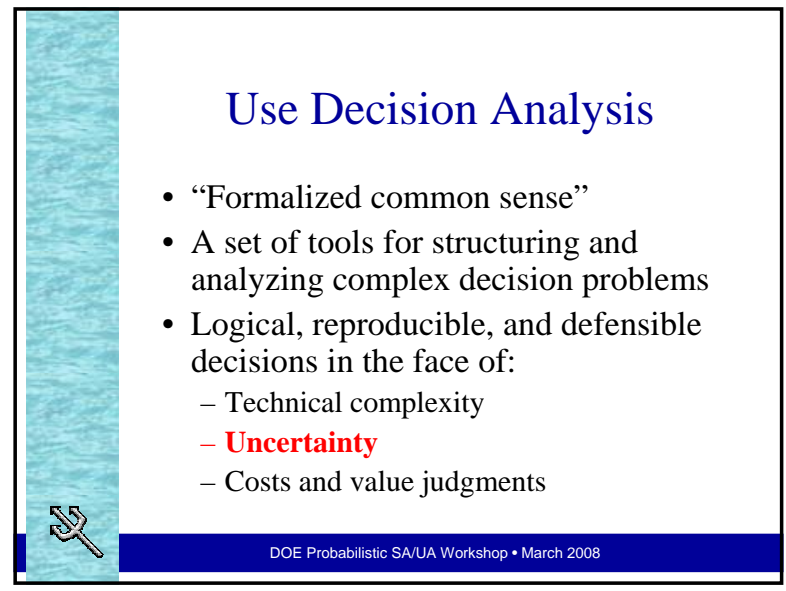

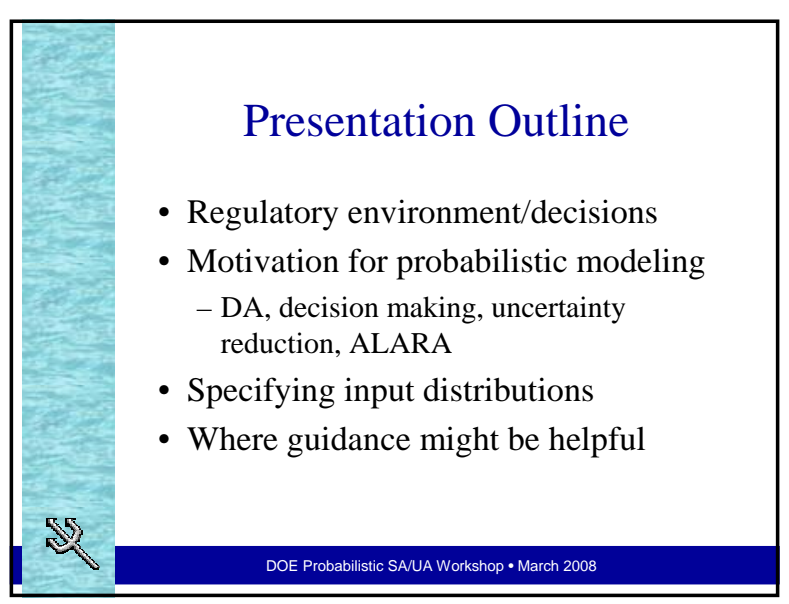
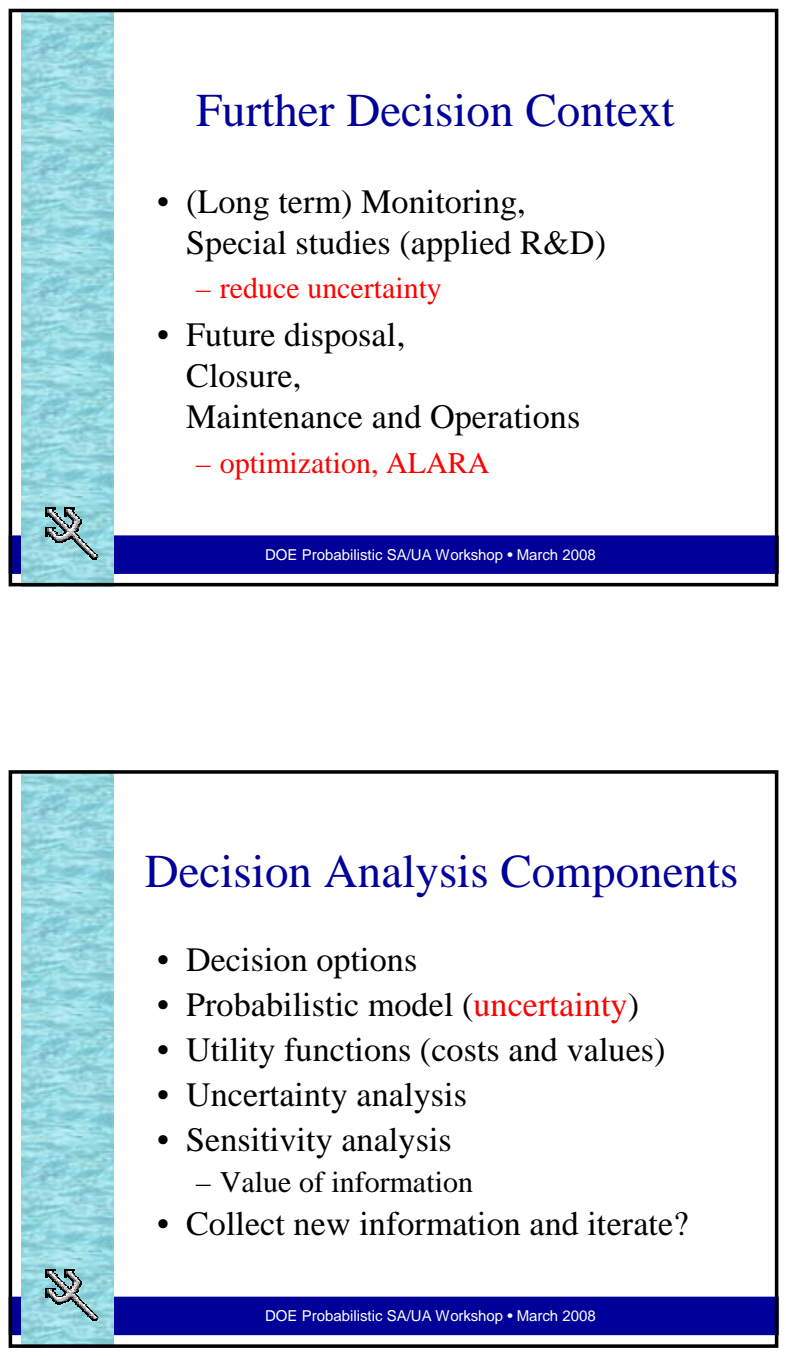


\section{Probabilistic Modeling}

- Model preparation

- Gather pertinent information

- Develop conceptual model

- Model structure

- Variables (parameters) and relationships

- Include alternative models, scenarios

- Model specification

- Probability distributions, correlations

- Conditions (assumptions)

- Costs, value judgments

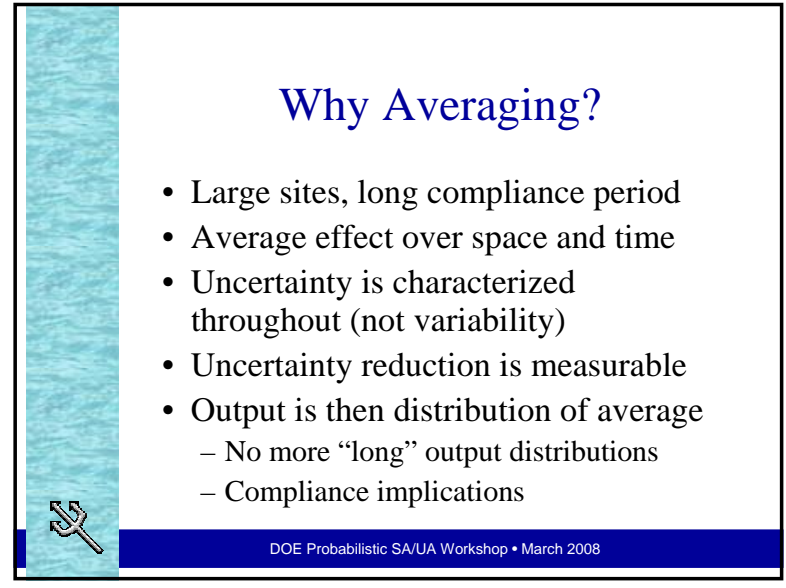

\section{Information Sources}

- Data

- Meta-data (relevant related data)

- Literature review data

- Other secondary data

- Surrogate data

- Other studies

- Model abstraction

- Elicitation
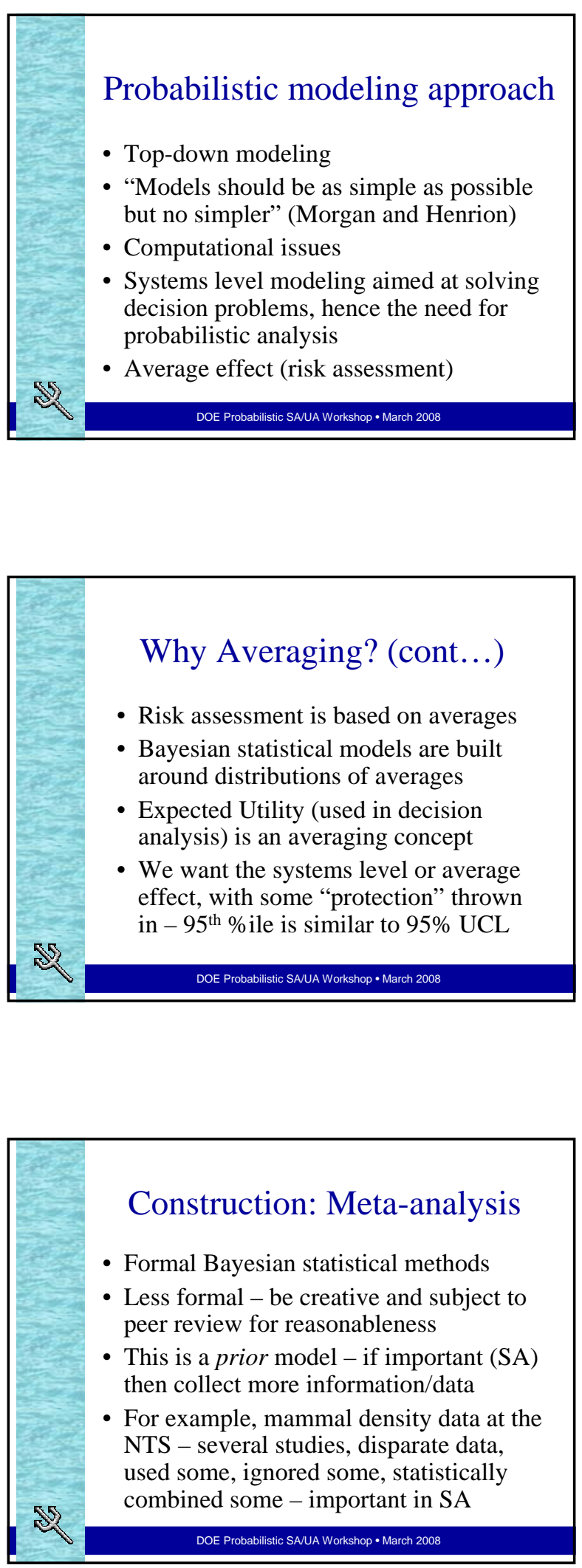

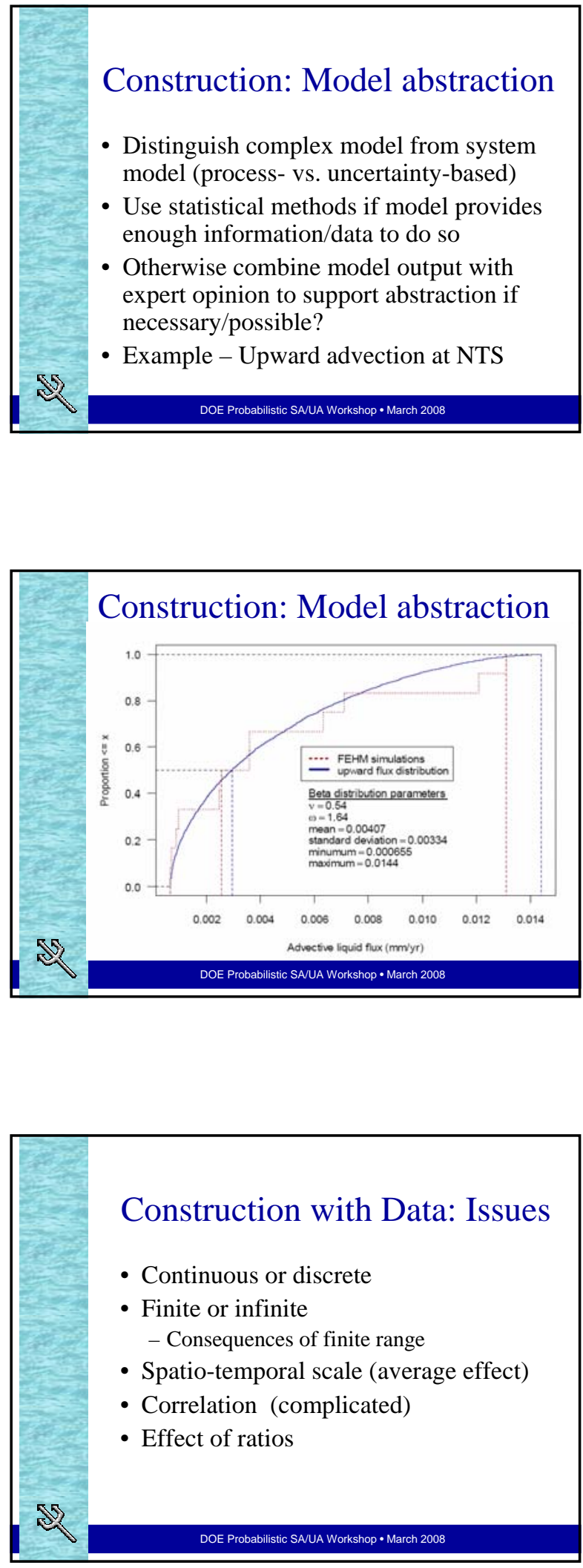

\section{Upward advection example}

- FEHM used to model gas and liquid phase flow and transport

- A range of fluxes for each pilot well was obtained by varying three parameters.

- root zone capillary pressure,

- time from pluvial to drier conditions,

- treatment of material properties as uniform or heterogeneous.

- 12 liquid and vapor flux profiles were fit using a beta distribution.

DOE Probabilistic SAUA Workshop • March 2008

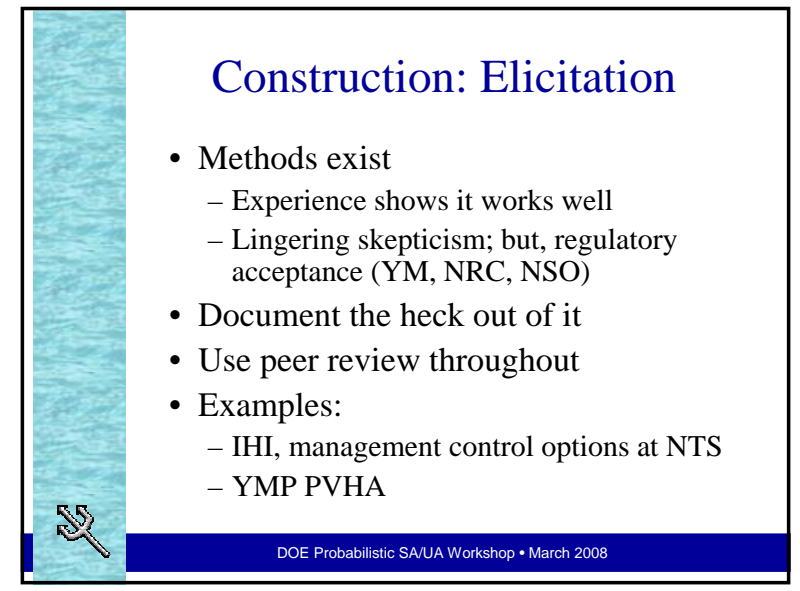

\begin{tabular}{|c|}
\hline Continuous vS. Discrete \\
\hline - Most PA variables are continuous \\
- When discretized, information is lost \\
and uncertainty is inadequately \\
characterized. \\
- For example: \\
- Inventory for the F-Tank Farm PA \\
\hline DOE Probabilistic SAUA Workshop March 2008 \\
\hline
\end{tabular}




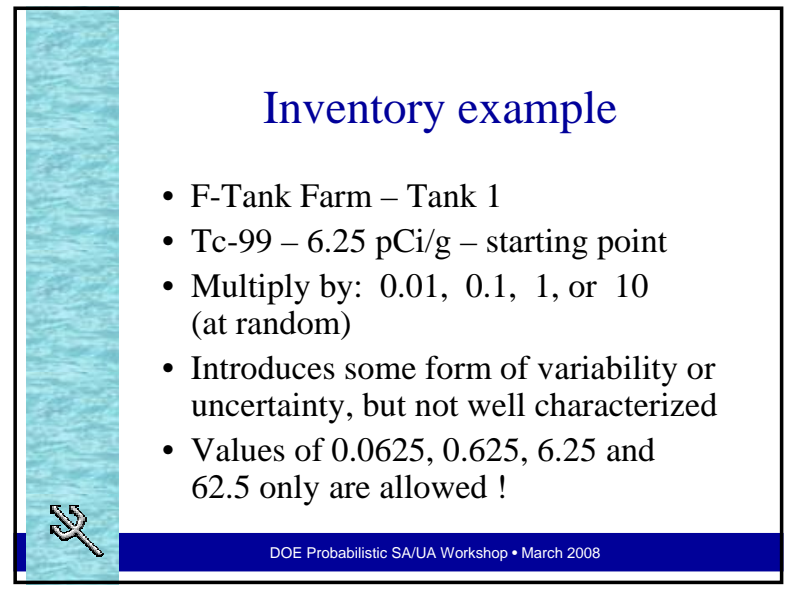

\section{Inventory example}

- Only 4 values are permissible.

- This is not reasonable - a value of 7 (pick a number) is just as reasonable

- $6.25 \mathrm{pCi} / \mathrm{g}$ was the best estimate

- But the average now is about $17 \mathrm{pCi} / \mathrm{g}$ - Not representative of our knowledge - Not properly characterizing uncertainty - Compromises UA/SA
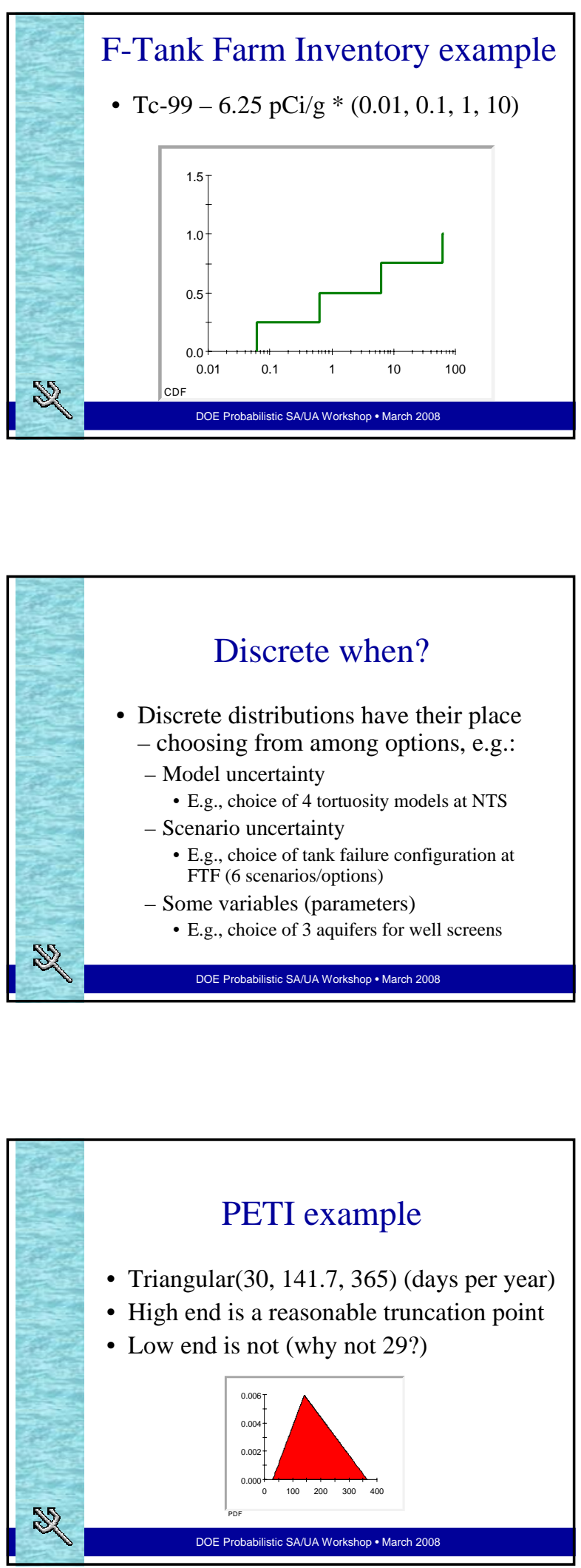


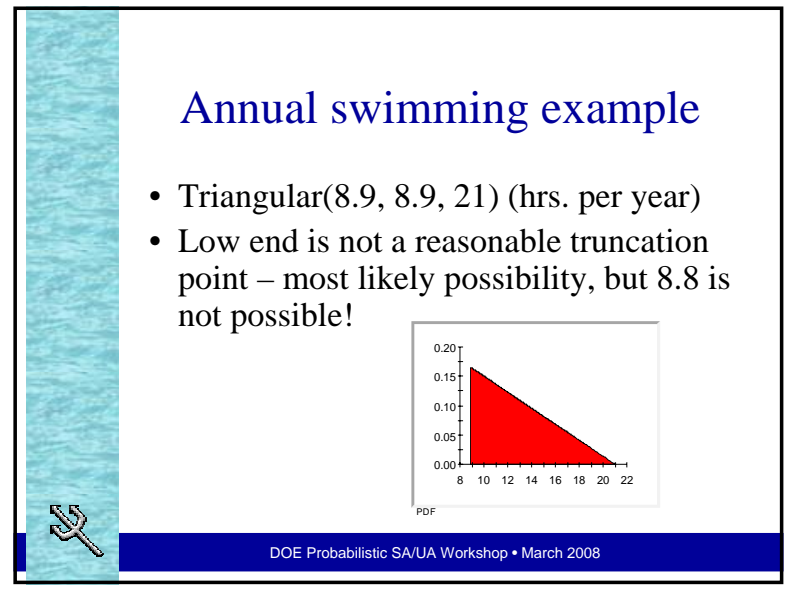

\section{Distribution of the average}

- Perhaps these would be better?

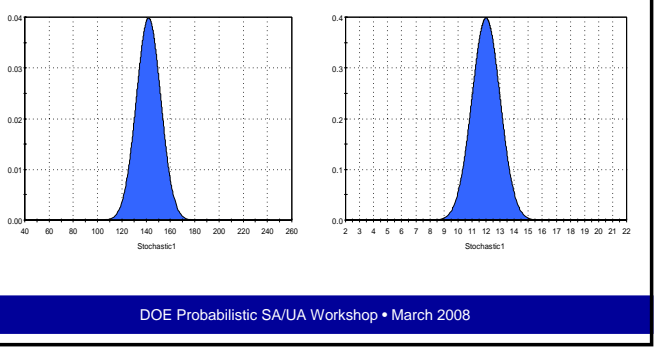

\section{Construction: Data}

- Distribution of average from bootstrapping data

- CLT is working for us with distributions of averages, so use it !

- Consider using Bayesian methods if data are minimal

- Example with lots of data

- Moisture content at NTS
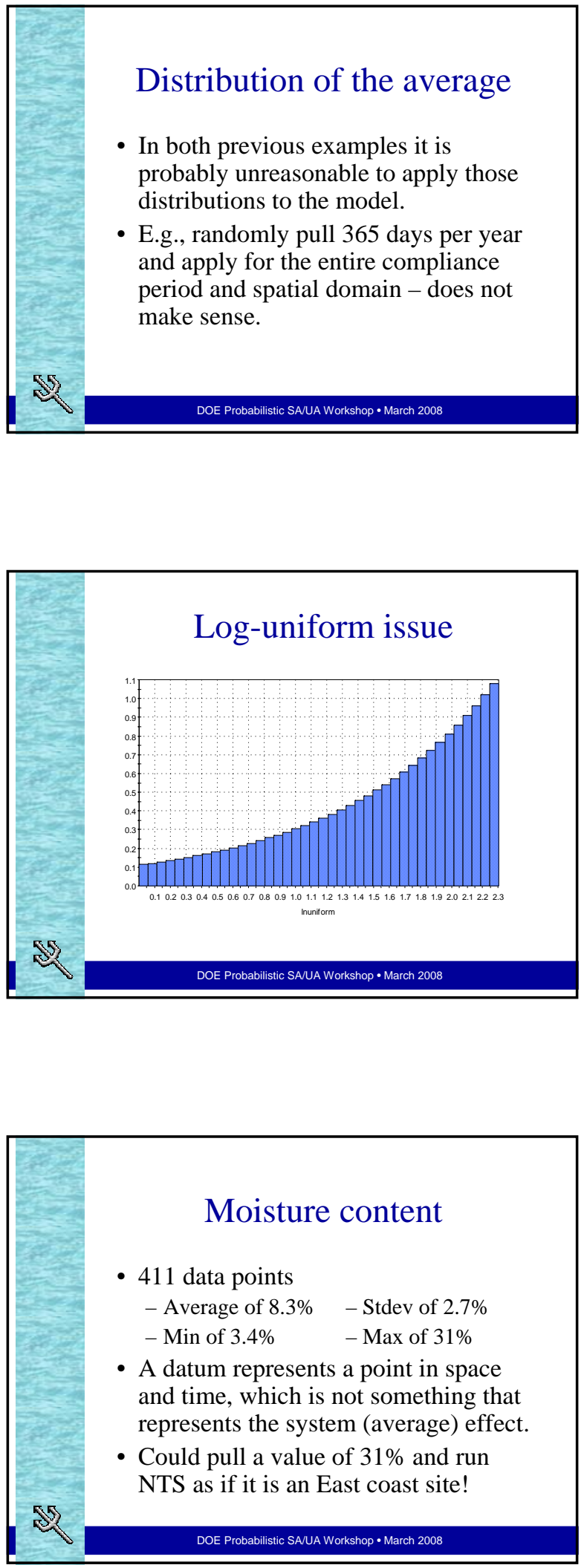

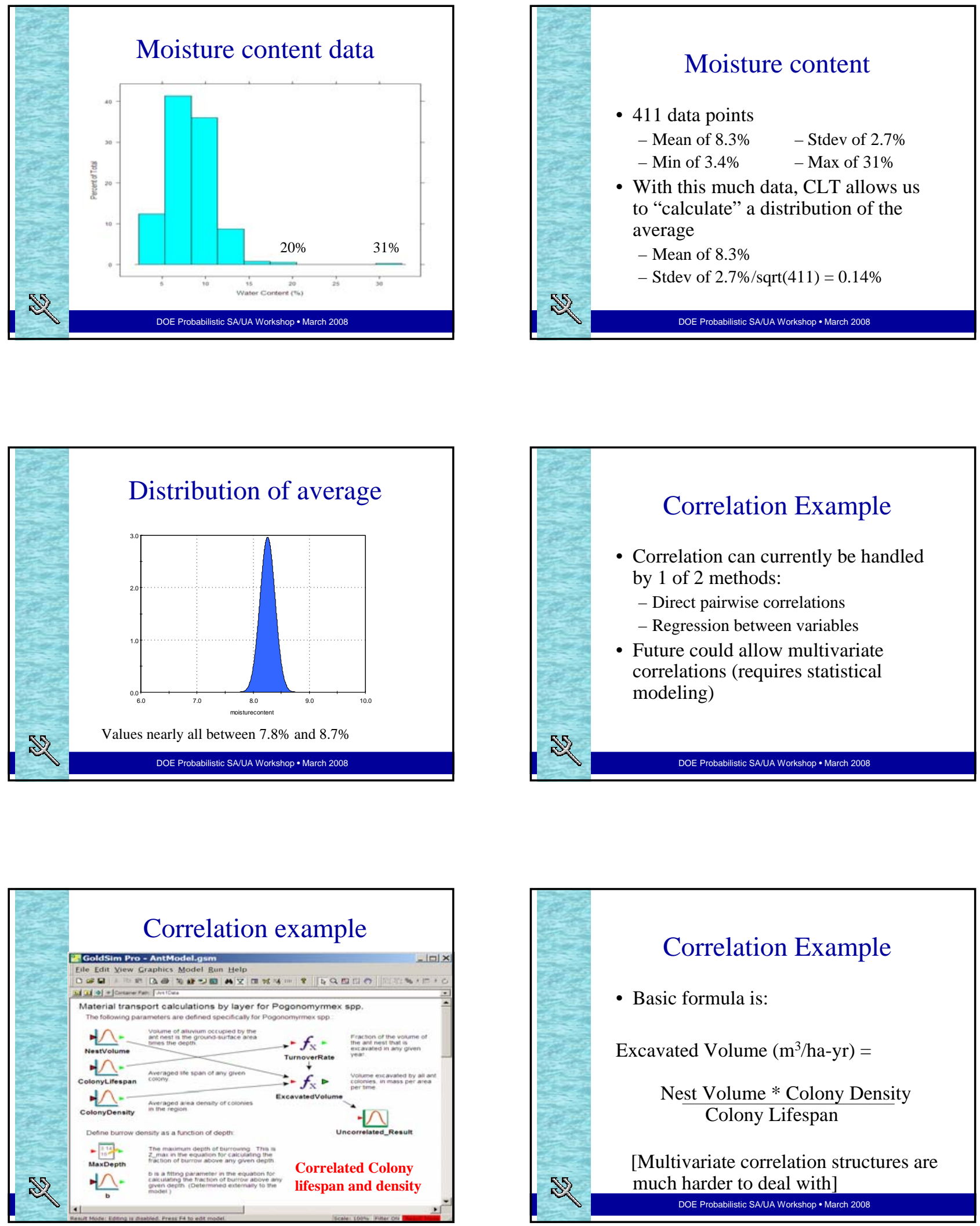

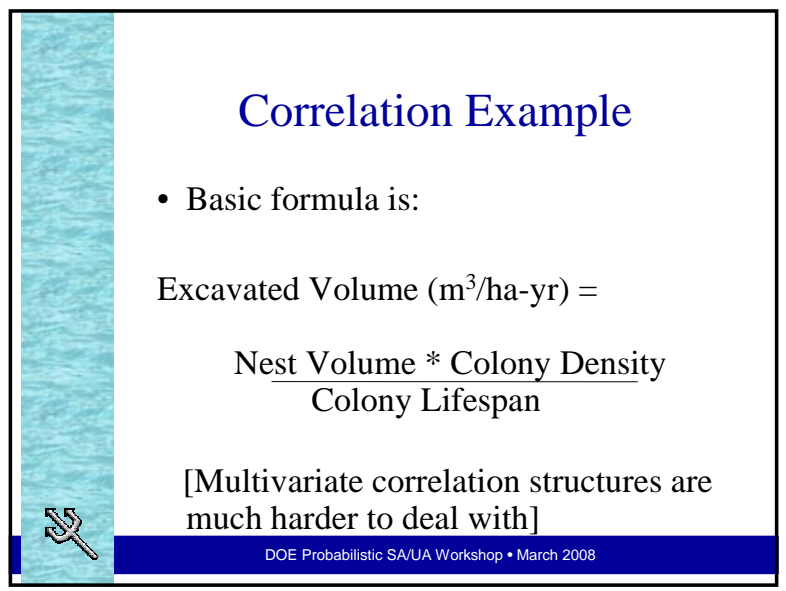




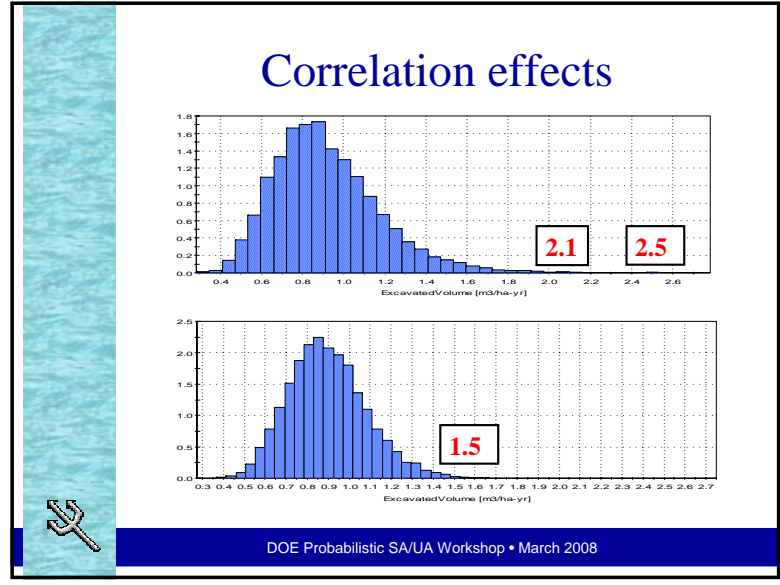

\section{Construction lessons}

- Lots of lessons learned

- Many stories to tell

- Statistical help is critical

- Find some statisticians you can talk to

- Who are not afraid of typically messy

environmental data sets and problems

- For success: Subject all input distributions to statistical rigor, documentation, peer review, openness

DOE Probabilistic SAIUA Workshop • March 2008

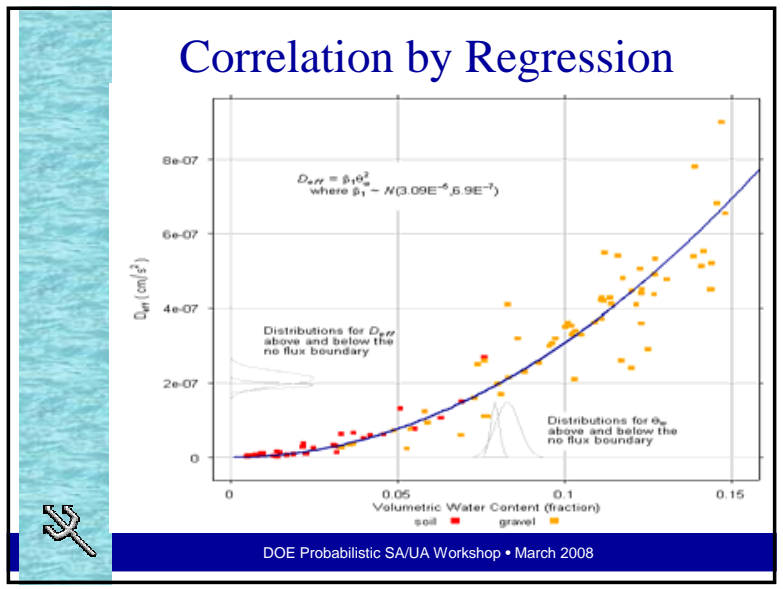

\section{If done properly....}

- Well specified input distributions lead to:

- Defensible decisions

- Useful UA, SA, and VoI

- Otherwise, all aspects of decision making are compromised

- Costs of taking unnecessary action

- Compromised SA

DOE Probabilistic SAIUA Workshop • March 200

\section{Environmental Evolution}

- Compliance using deterministic models

- Difficult to overcome inertia and intransigence in the industry (old dogs new tricks)

- Difficult to overcome established regulations

- Changes at the top-level take a long time to trickle down

\section{Environmental Evolution}

- Successful history of compliance decisions using deterministic models

- Most regulations are deterministic

- Conservatism distorts and/or hides uncertainty of decisions

- Short-circuits continuing data exploration and iterative modeling cycle 


\section{Environmental Evolution}

- Deterministic modeling continues to be useful for exploring processes of fate and transport

- Augmented with probabilistic modeling allows quantification of uncertainty and more effective decision making

- This was difficult 20 years ago, but is very possible now

- But still a continued reluctance to change (old dogs - new tricks?)

DOE Probabilistic SAUUA Workshop • March 2008

\section{PA/CA Application}

- Probabilistic decision-based modeling becomes a decision tool to evaluate decision objectives

- Compliance

- Future disposal

- Closure

- Monitoring

- Maintenance and Operations

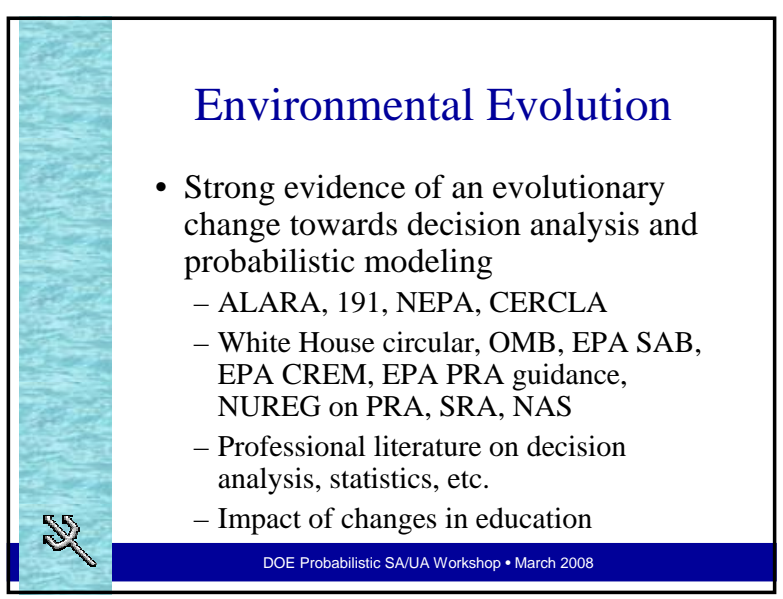

\begin{tabular}{|l|}
\hline PA/CA Application cont... \\
- DA becomes defensible \\
- SA becomes more effective at \\
identifying important variables \\
- SA performed simultaneously for all \\
input variables \\
- Models must be based on expectation \\
and uncertainty to avoid compromised \\
decision analysis process. \\
Doe Probabilistic salua workshop March 2008
\end{tabular}

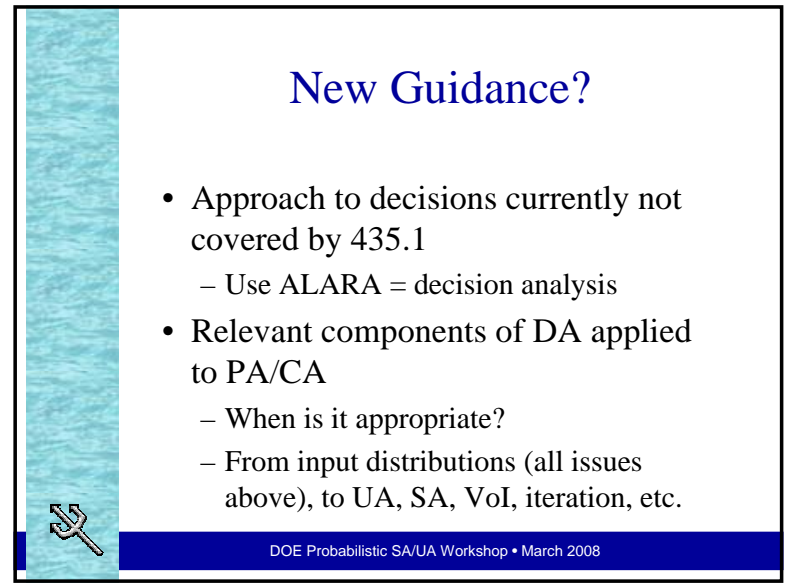


WSRC-STI-2008-00333, REVISION 0

\section{SESSION 2 - MODEL ABSTRACTION}


WSRC-STI-2008-00333, REVISION 0

This page intentionally left blank. 


\section{Abstraction Session Overview}

\section{- E-Area PA}

- Case study involved reducing dimensionality only

- 1D flow fields inferred from 2D UZ and 3D SZ simulation results.

- Provided discrete flow fields to be built into GoldSim.

- Compare PORFLOW transport simulations to calibrate GoldSim

\section{Session Overview}

- All modeling should have clearly defined objectives. The objectives can influence the need for and approach to abstraction. (Rope example)

- An important reason for model simplification is improved communication with stakeholders

- The detailed and simplified models can be complementary.

- Abstraction can be difficult, but simplifications can be sometimes be made. Sometimes not.

- What comparison criteria should be met to accept abstraction?

\section{Session Overview}

- MDA G PA

- Abstraction of flow and transport in a complex hydrogeologic setting

- Developed a 3D model to estimate breakthrough at the compliance boundary

- The model incorporates available data and previous modeling

- Examined system behavior with 3D model. Identify less important features/factors

- Systematic abstraction process 3D pathways to 1D pathways

\section{Session Overview}

- Dimensionality reduced without changing flow and transport behavior

- Comparison of $1 D$ results with 3D simulations

- Linked to GoldSim through DLL for flexibility

- Abstraction of flow, reactive transport, and well capture

- Abstraction makes MC simulations practical

\section{Session Overview}

\section{- Model Abstraction and Treatment of Model} Uncertainty

- Importance of alternative conceptual models

- Model abstraction is part of a comprehensive approach to model uncertainty 

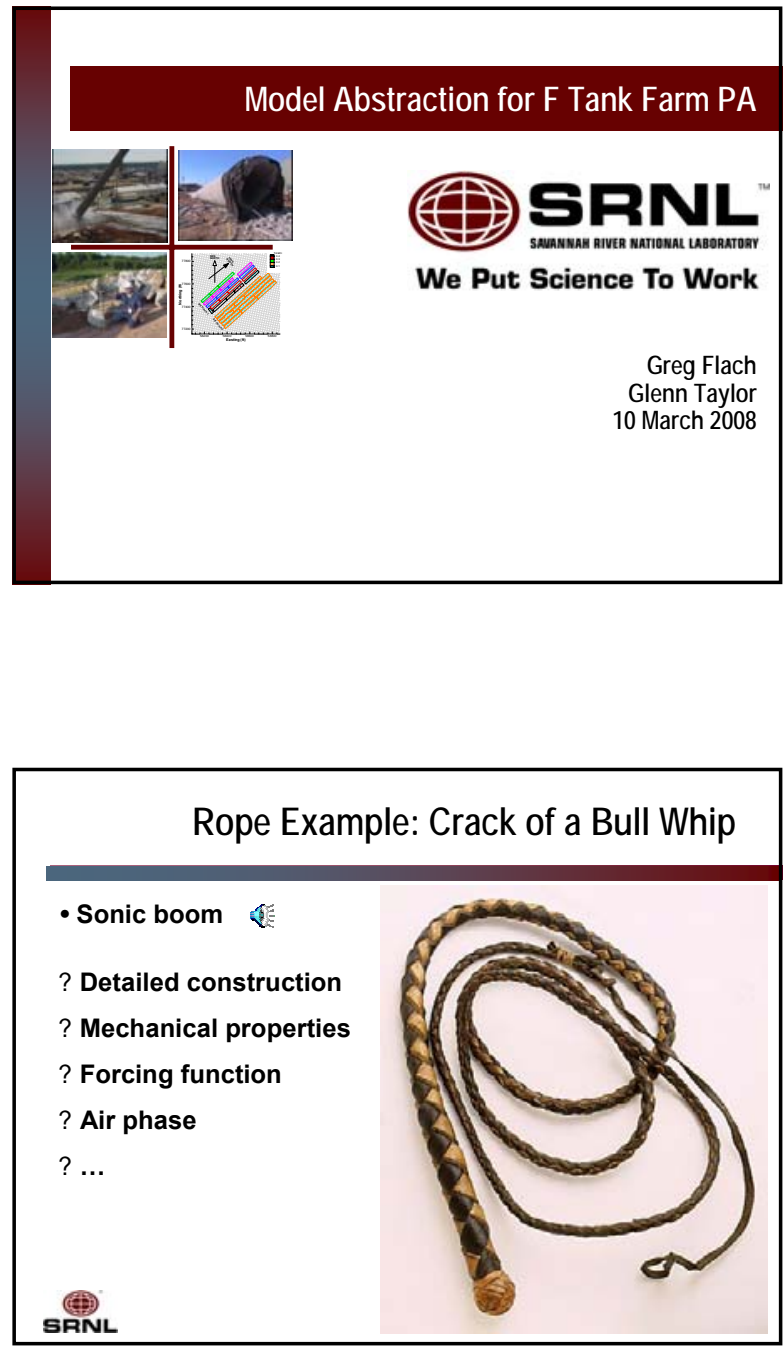

\section{Settings Conducive to Model Abstraction}

- Stakeholders often focus on simpler representations in making decisions or gaining understanding

- not subject matter expert?

- limited time to understand complex model?

- management of uncertainties?

- more straightforward communication?

- detail obscures essence?

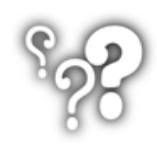

\section{Technical Challenges to Model Abstraction}

- Multi-dimensional phenomena

- Sensitivity to small-scale features

three-dimensions, detail

- Non-linear / chaotic behavior

- Competing effects / mechanisms

full physics

SRNL

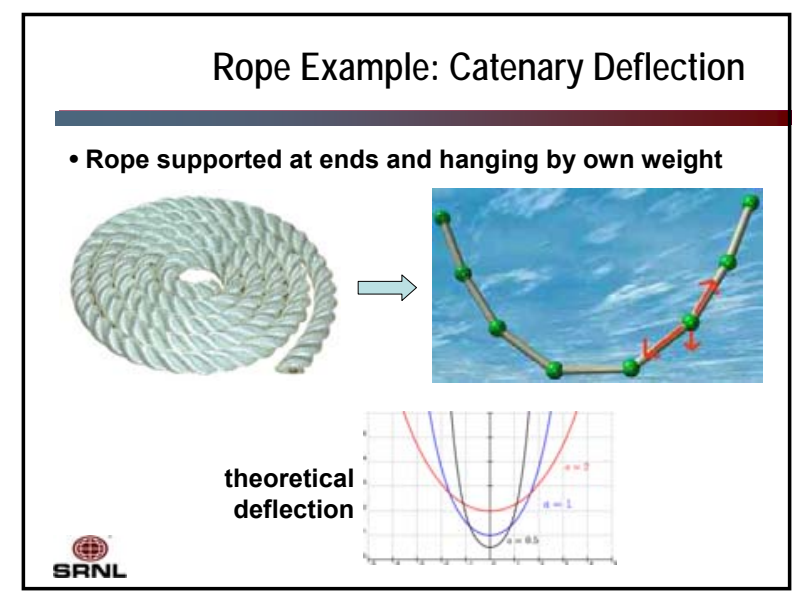

\section{Settings Conducive to Model Abstraction}

- Weak link in overall analysis is often

- choice of conceptual model / scenario definition

- existence of competing alternatives

- insufficient data

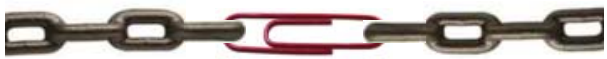

(4)

SRNL 


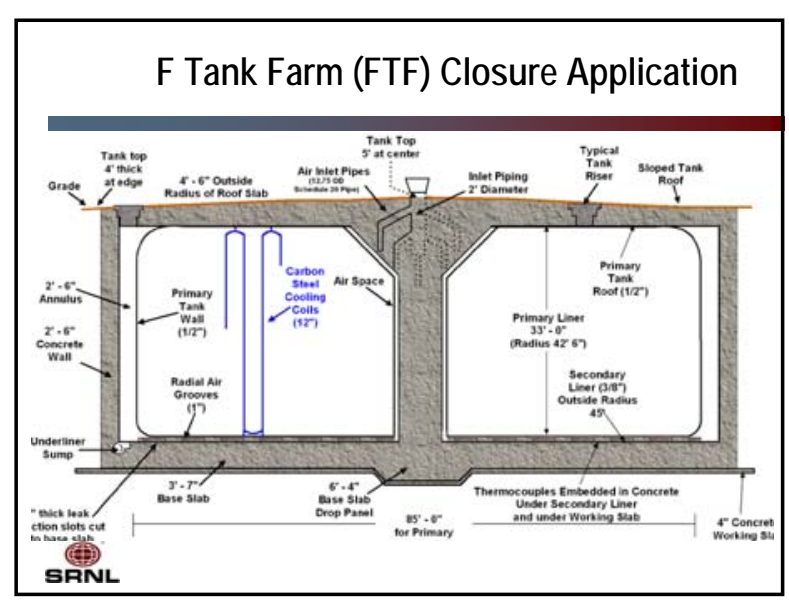

\section{FTF Performance Assessment}

\section{- Technical challenges}

$\cdot 10,000+$ year period of performance

- Degradation of cementitious materials not well understood

- Data on long-term durability lacking

$\rightarrow$ Significant uncertainty in failure scenarios and input data

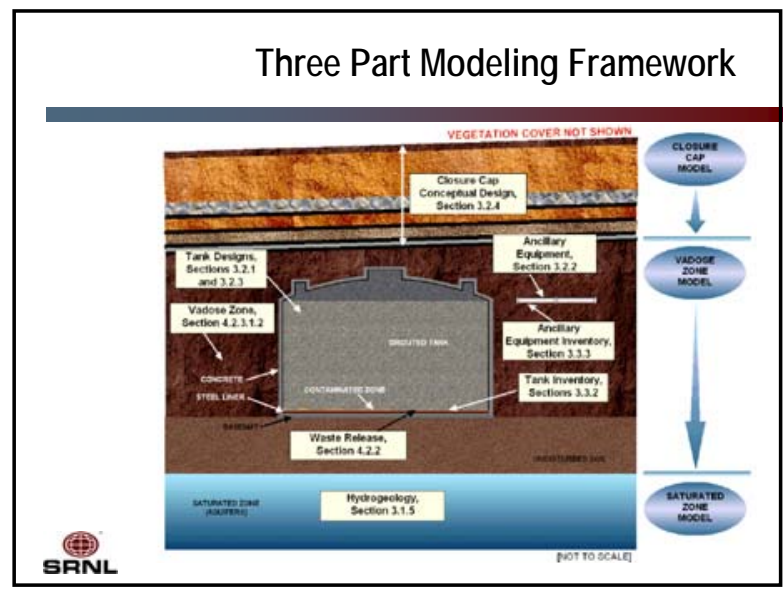

\section{FTF Performance Assessment}

- Stakeholders
- DOE
- SCDHEC
- EPA
- NRC
- Public
$\rightarrow$ Wide range of technical expertise and information
needs
SRNL

\section{Porflow and GoldSim Subsurface Transport}

- Chose simple abstractions of actual system through appropriate scenario definition

- Hybrid quantitative transport modeling approach:

- Porflow-based model for fuller physics

- multi-dimensions, detail

- GoldSim-based model for reduced physics

- one-dimension, coarser

- Reality $\rightarrow$ Scenario abstraction exceeds

Porflow $\rightarrow$ GoldSim abstraction

SRNL

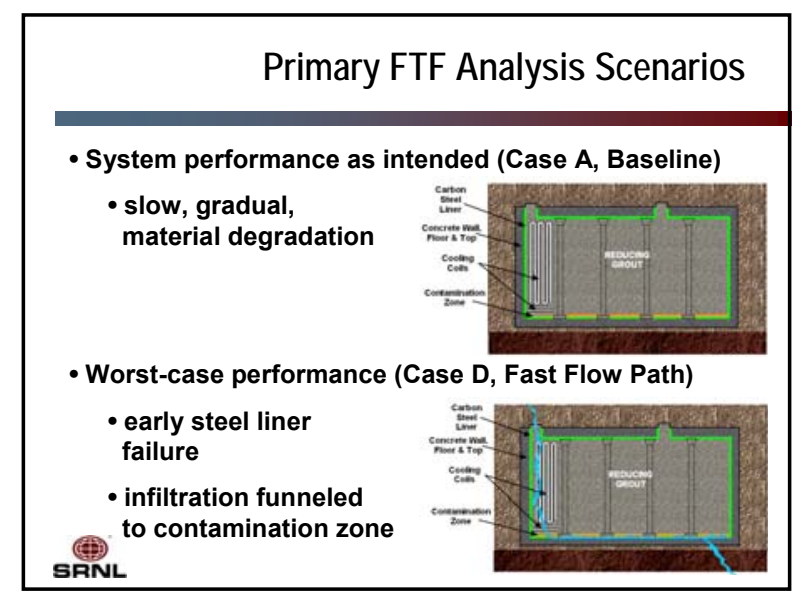




\section{Porflow to GoldSim Abstraction}

-Vadose zone transport

- 2D Porflow flow field $\rightarrow$ 1D GoldSim flow field

- Physical flow is essentially 1D for Case A and D

-Aquifer transport

- 3D Porflow flow field $\rightarrow$ 1D GoldSim flow field

- 3D transport (using GoldSim "plume" function)

- Other physics preserved or enhanced

$\rightarrow$ some aspects of multi-dimensional flow sacrificed $\Leftrightarrow$
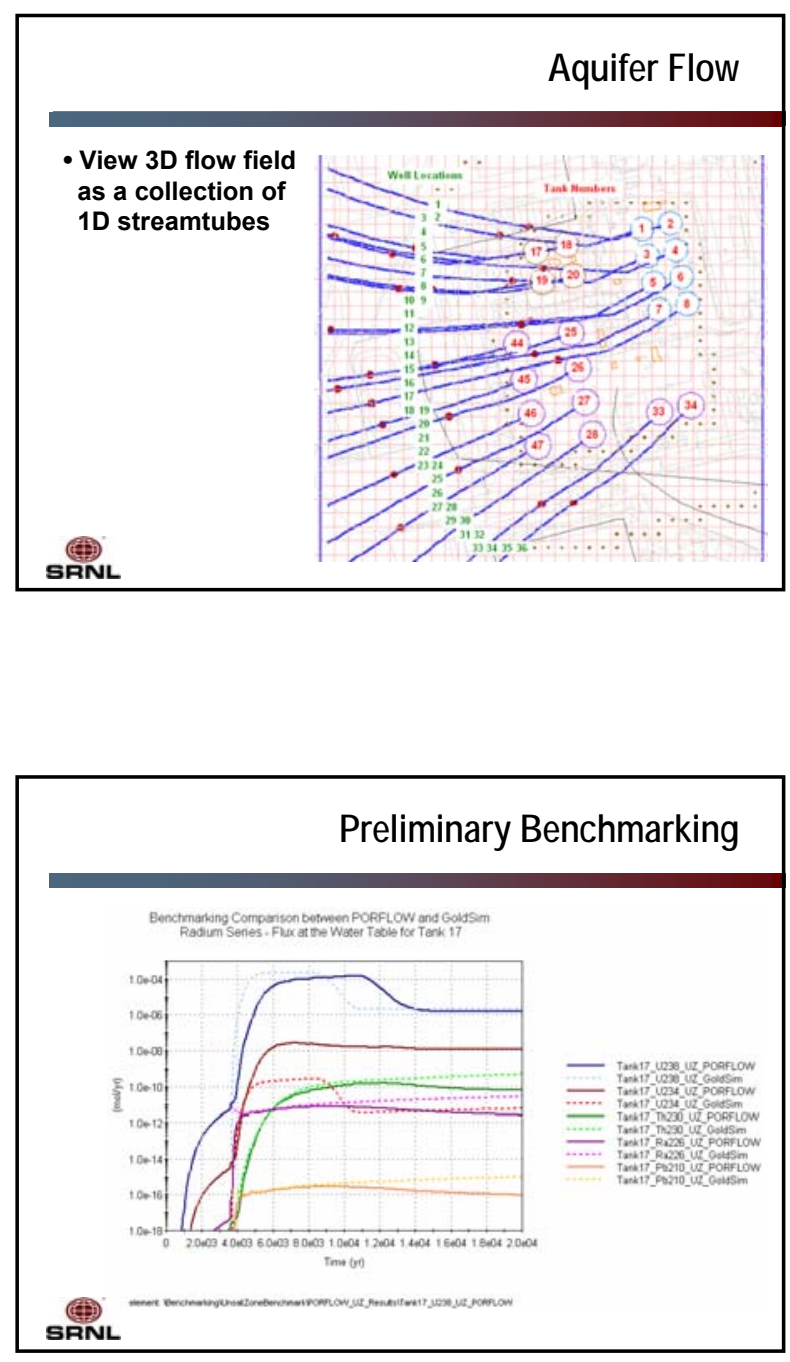
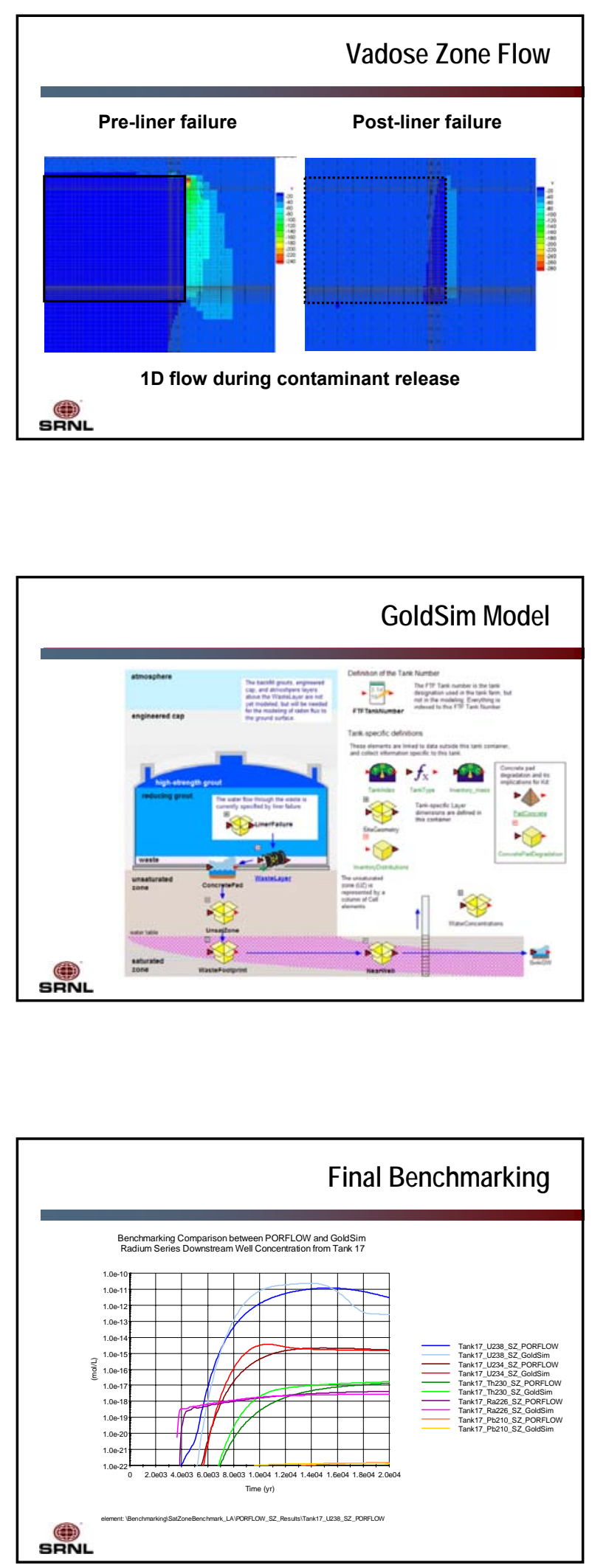


\begin{tabular}{|l|}
\hline Benchmarking Considerations \\
- Using hybrid approach means differences must be \\
explained \\
• Have to understand bumps and wiggles in all the \\
models, \\
• but not necessarily match every aspect of results \\
- Both models should also be validated against reality \\
•What is a reasonable comparison? \\
SRNL
\end{tabular}

\section{Key Points and Questions}

- Simplify when prudent (otherwise don't)

- Consider non-technical drivers for simplification

- Better yet ... hybrid approach

(full \& reduced physics are complementary)

- Be persistent ... sound abstraction not easy

-What level of agreement is sufficient?

SRNL

\section{Advantages of Hybrid Approach}

- Independent QA check

- More complete physics (collectively)

- e.g. solubility controls

- Explaining differences forces better understanding

- Responsive to deterministic and probabilistic

"camps" among stakeholders

- Benchmarked Goldsim model enables quick response

to future issues (e.g. Special Analyses)

SRNL 


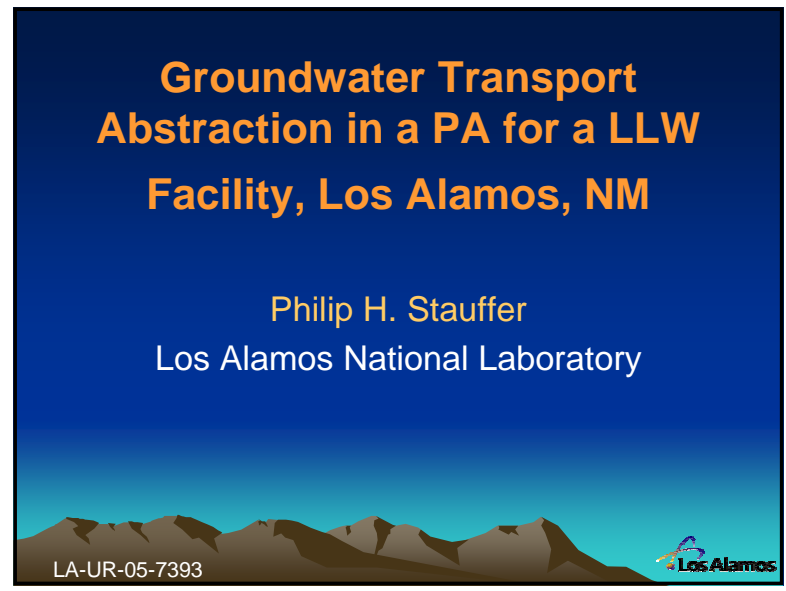

\section{Talk Outline}

1) MDA G Background and Data

2) 3-D ground water model for MDA G

3) Reduce complexity of 3-D model

4) Link to probabilistic PA model

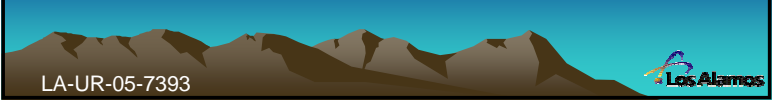

\section{Material Disposal Area G}

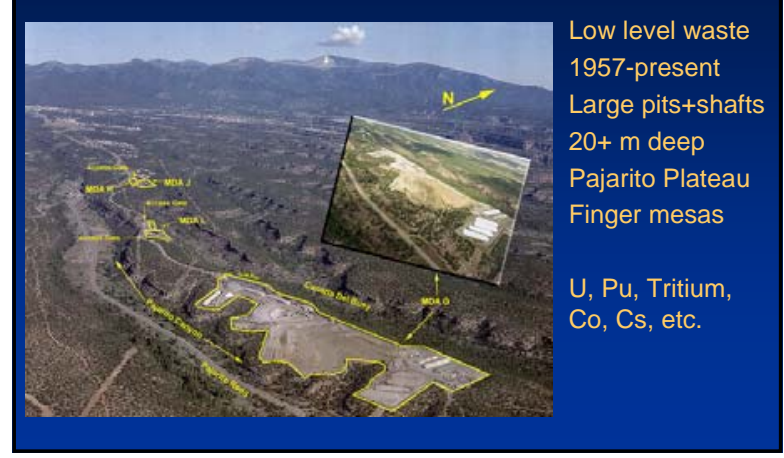

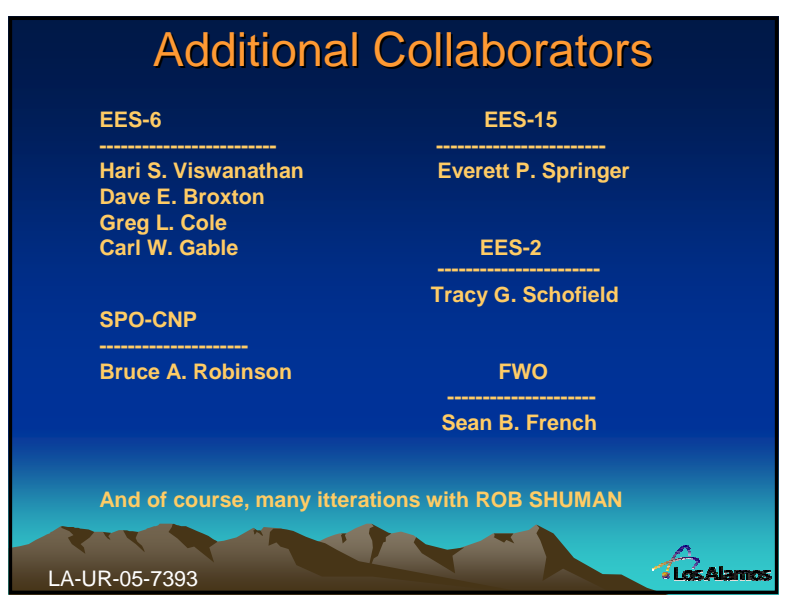

\section{Part I}

MDA G Background and Data

\section{Extensive Data}

1) Characterization over many years

2) Dozens of boreholes

3) Previous PA provided insight and data

4) Many modeling studies around LANL

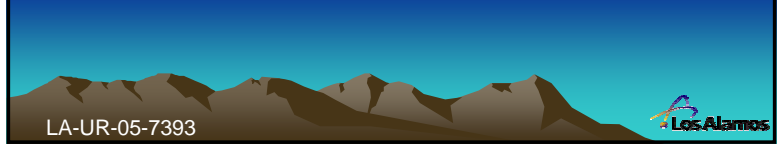



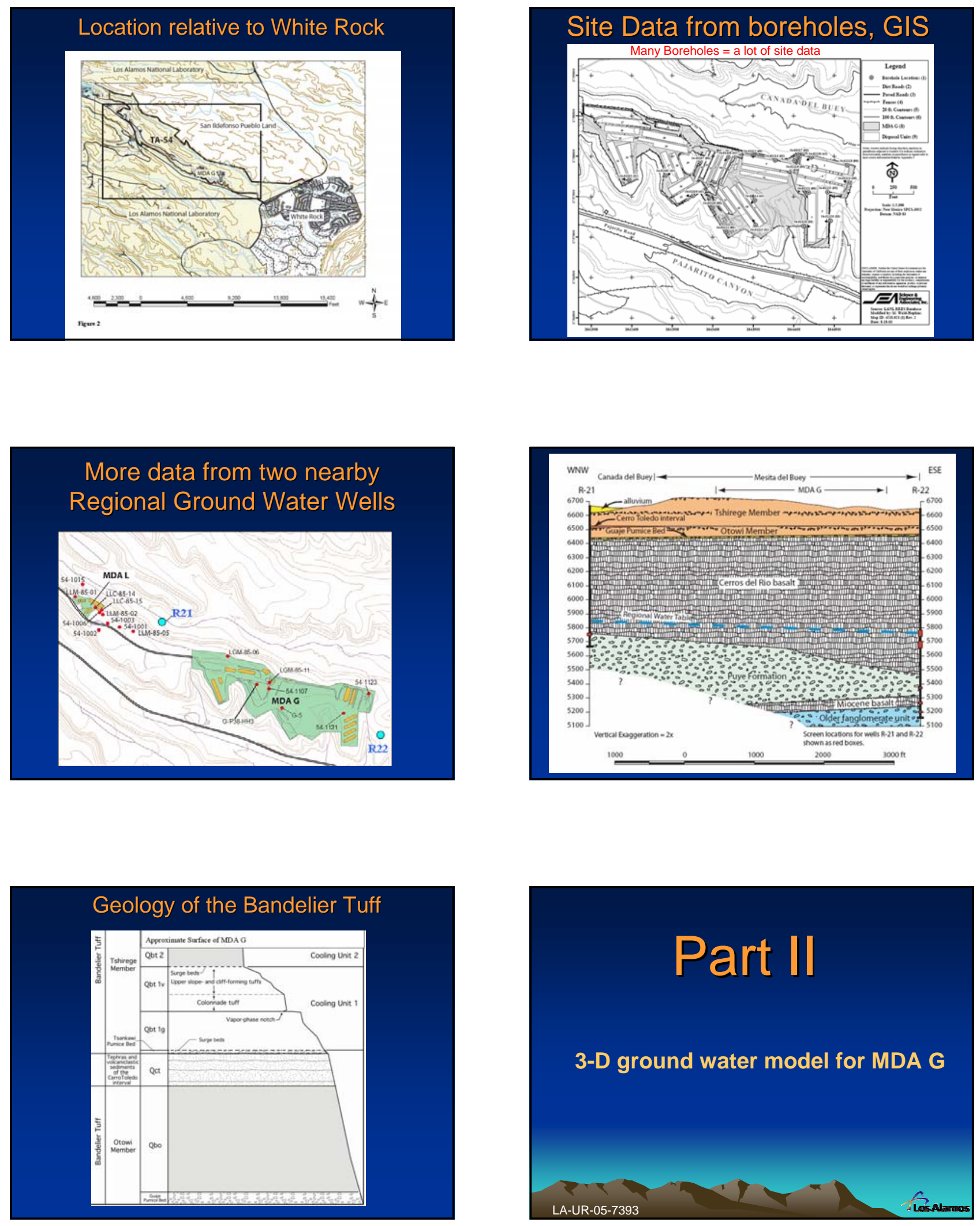


\section{3-D Model}

- Calculates groundwater flow and transport

- Output $=$ Breakthrough at compliance boundary

- Incorporates available data and previous modeling

- Covers an area much larger than the site

- Includes many rock types, topography, dipping beds

- Tests conceptual model hypotheses

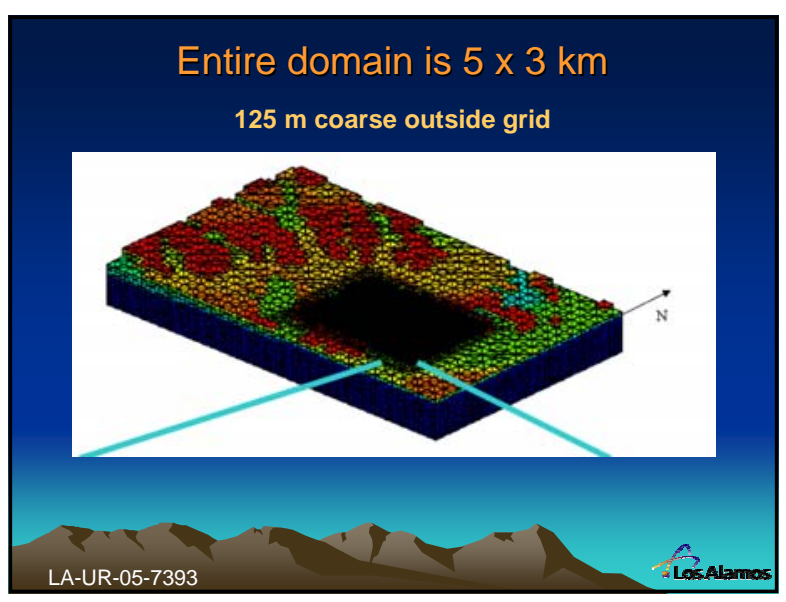

Zoom in on the MDA G Mesa Edge $6.5 \mathrm{~m}$ grid

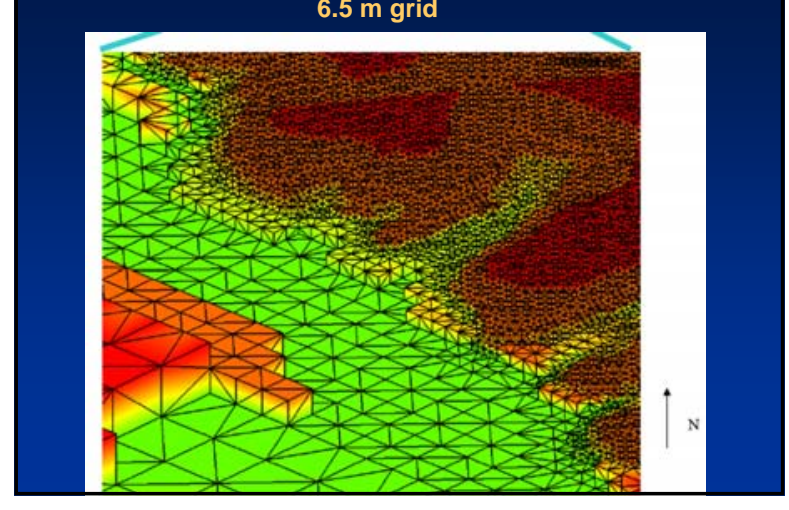

Surface Saturation Example

Wet Pajarito Canyon

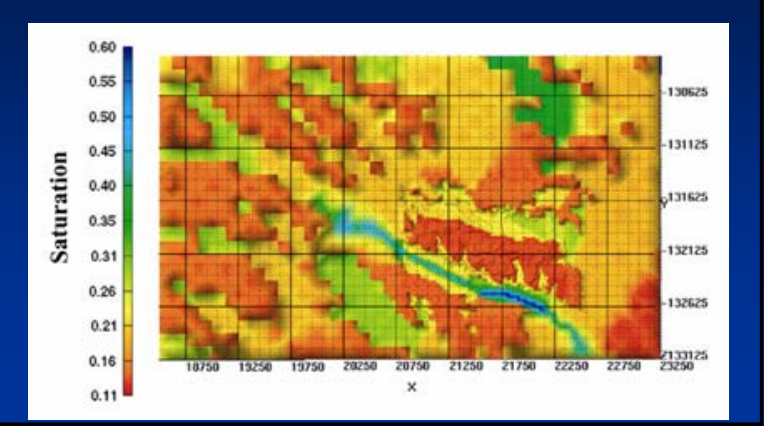

\section{Numerical Simulator FEHM}

- Los Alamos code: finite volume, multiphase, heat and mass transfer in porous media

- Capable of simulating vapor transport processes, phase partitioning, air convection, reactive chemistry

- Wellbore capability embeds 2-D radial boreholes in existing 3-D grids

- Extensively used for YMP, WIPP, LANL ER, CO Sequestration, Hot Dry Rock, Methane Hydrate

\section{Part III}

Reduce complexity of 3-D model 


\section{3-D Analysis can reduce probabilistic needs}

- Can rule out scenarios

- Can put bounds on system behavior

- Most defensible approach, can include high resolution interfaces and complex processes

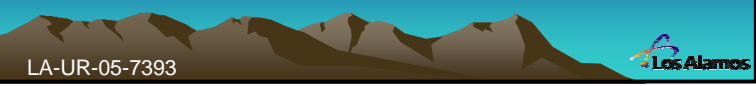

\section{Locations of 8 Pit/Shaft Clusters}

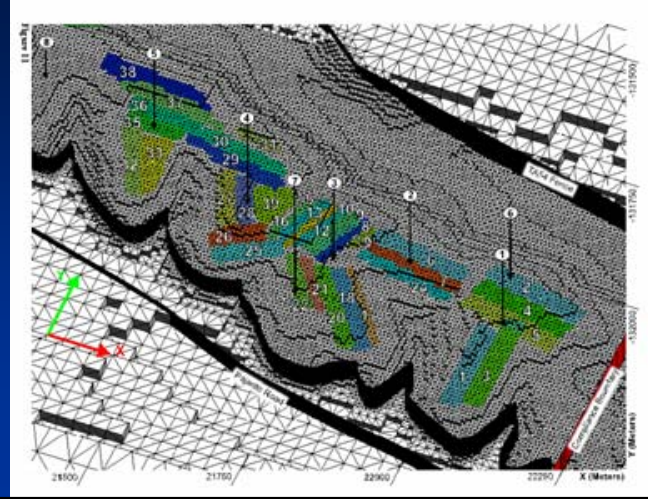

\section{Use Particle Tracking}

- Fast simulations

- Generate general flow path and timing

- Particles have no numerical dispersion

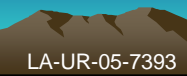
Losalames

\section{Analyze Limited Pathways}

To the Compliance Boundary

- 10 Infiltration Fluxes (0.1 - 10 mm/yr)

- Generate steady state 3-D flow field

- 8 Release Locations

-6 Pit Clusters $20 \mathrm{~m}$ deep

- 2 Shaft Clusters $60 \mathrm{~m}$ deep

80 combinations to simulate

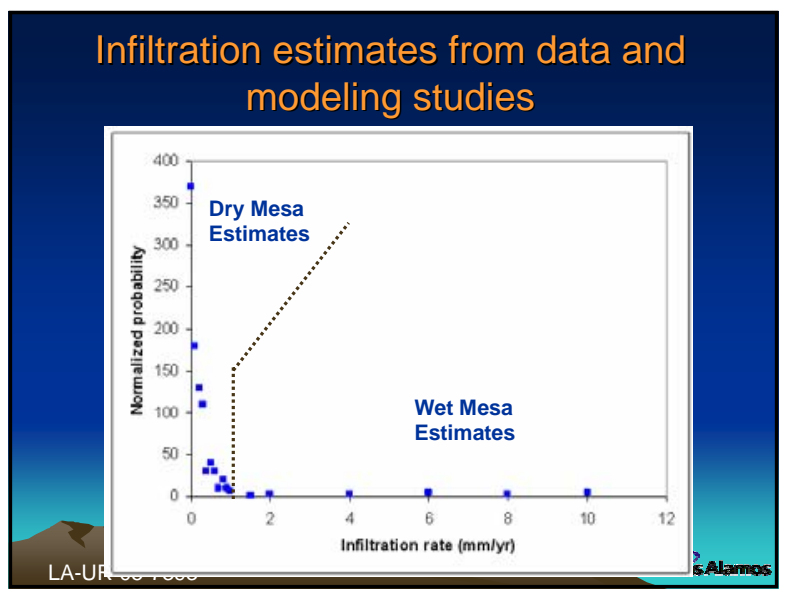

3-D Scenario Testing Example

Wet versus Dry Pajarito Canyon : No impact

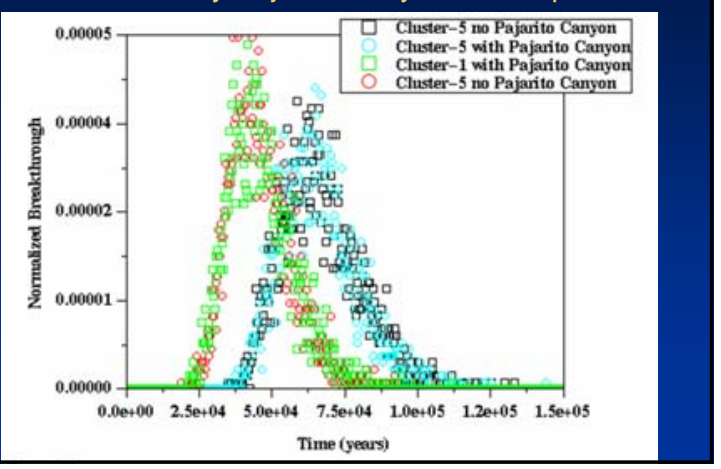


3-D Model Sensitivity to Release Area

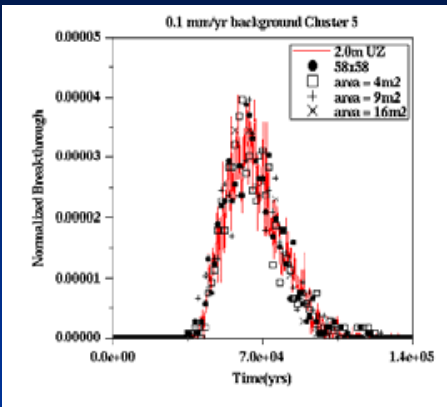

Release area is not important for PA

\section{System Behavior Example}

Thicker UZ = Slower particles

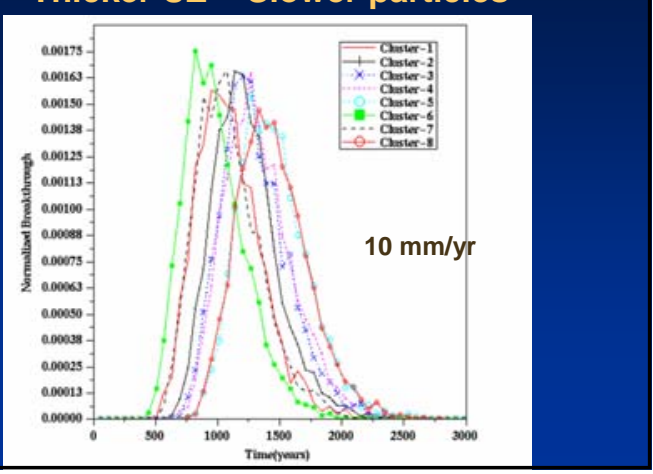

\section{Part IV}

Link 3-D results into a probabilistic PA model

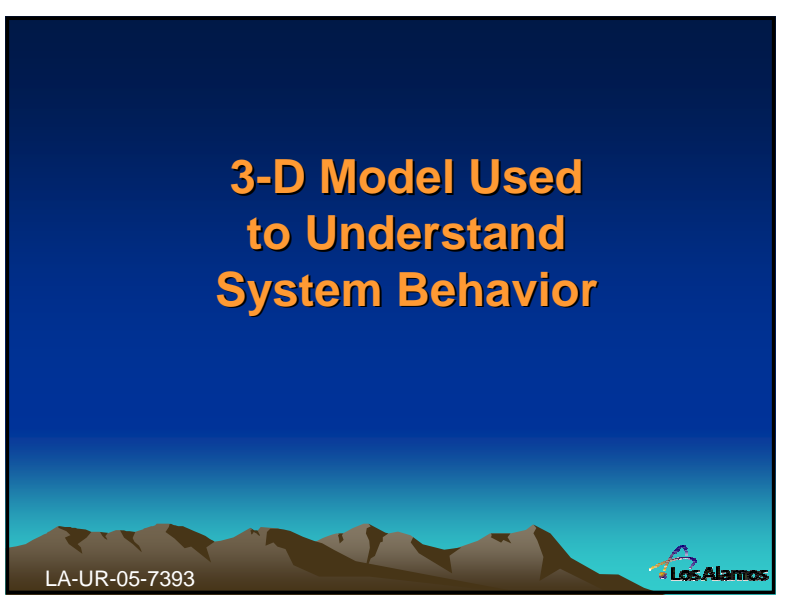

\section{System Behavior Example}

Lower infiltration $=$ Slower particles
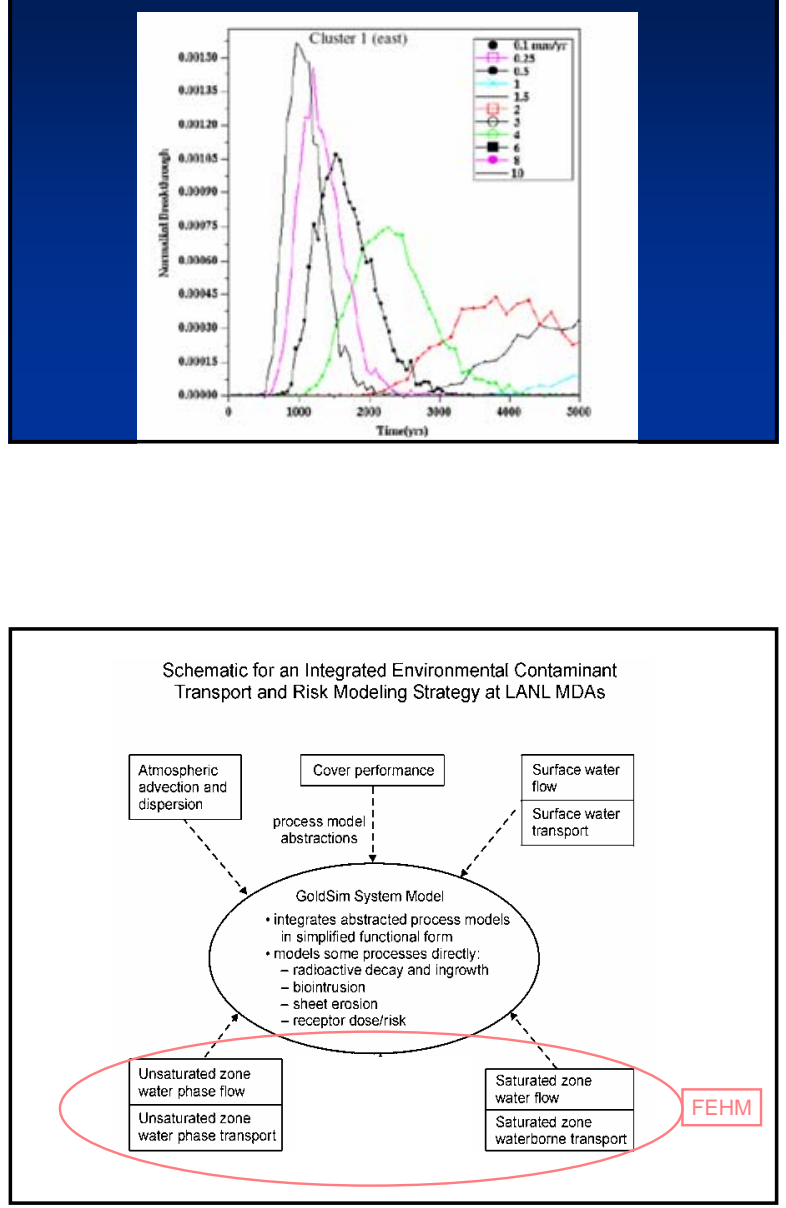


\section{Abstraction Details}

- Convert 80 3-D pathways to 80 1-D Pathways - Micromixing theory

-1-D pathways capture decay, ingrowth, sorbtion

- GoldSim samples

- Sorption $\mathrm{K}_{\mathrm{d}}$

- Infiltration rate

(tuned to 3-D model)

(discrete sampling)

- Inventory

(estimates for pit clusters)

- GoldSim gives contaminant mass flux to FEHM - 8 simultaneous FEHM 1-D simulations

- FEHM returns mass flux to GoldSim

\section{Abstraction Assumptions}

- To first order, travel time is not affected by uncertainty in hydrologic properties (porosity, saturation, bulk density)

- Mass flux returning to GoldSim hits the water table with the footprint of the overlying cluster

- Subsequent flow to a drinking water well at the compliance boundary is a second abstraction

\section{Ground Truth 1-D vs 3-D}

Not exact matches but does capture general behavior

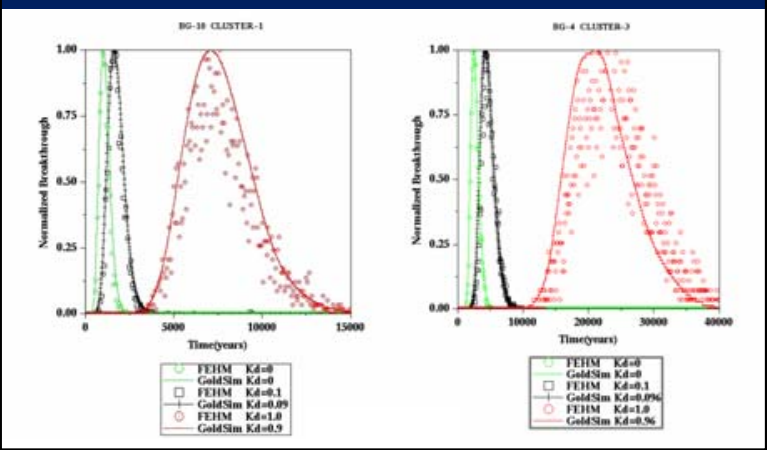

FEHM Micromixing Models: Macro RTDM

$\mathrm{C}_{\text {in }}$

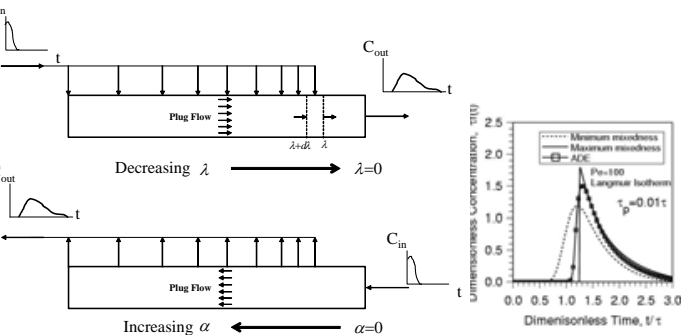

Robinson, B. A., and H. S. Viswanathan, 2003. Application of the theory of micromixing to groundwater reactive transport models, Water Resour. Res. 39(11), 1313, doi:10.1029/2003WR002368.
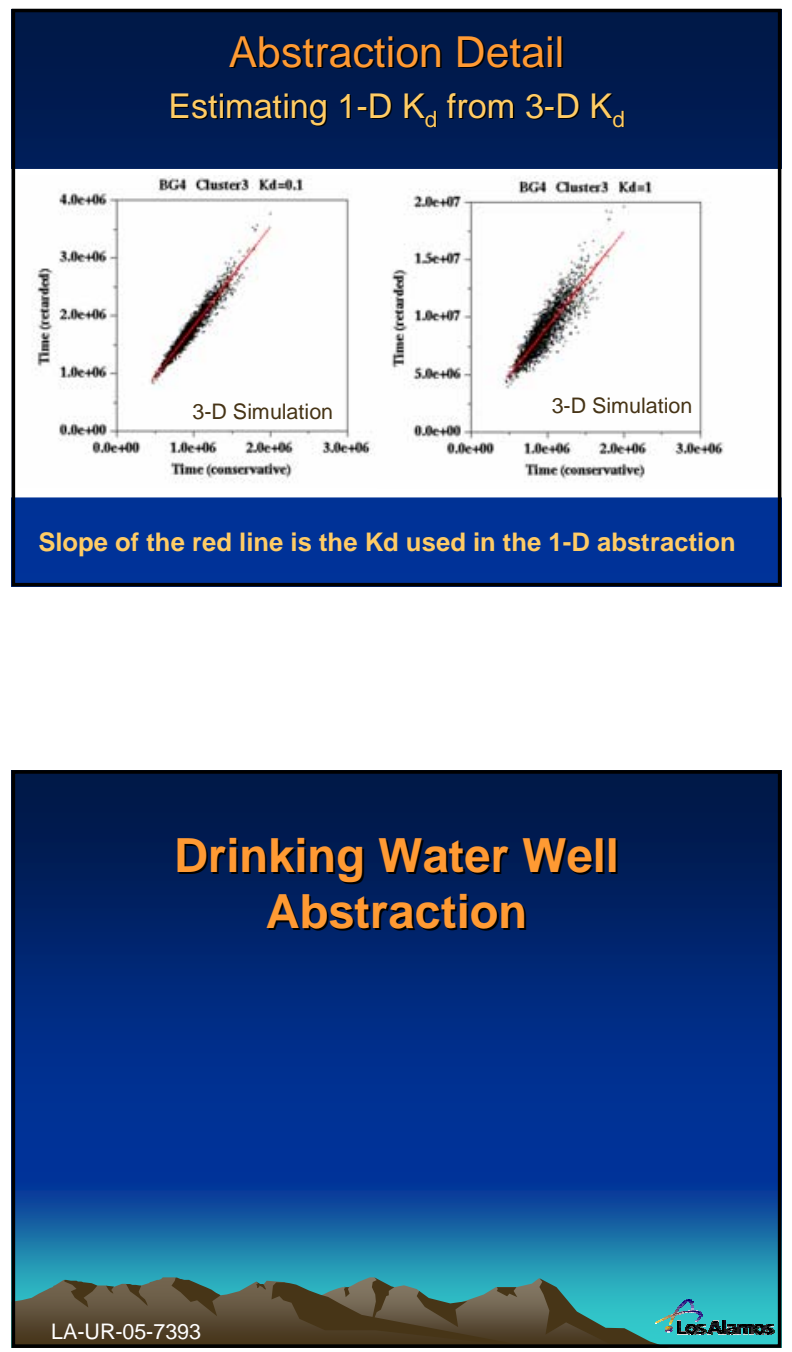
Example of Mass Arrival Footprint at the Regional Aquifer

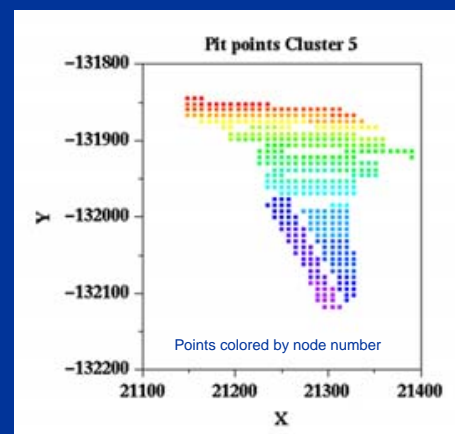

\section{Conclusions}

- 3-D ground water model can be abstracted to capture $1^{\text {st }}$ order effects

- Model abstraction takes time, but is especially useful to make PA calculations tractable.

- PA requires 1000 s of realizations for each run

- may need to be run 100 s of times to fix bugs in all submodels etc.

- Reduction in complexity must be defensible
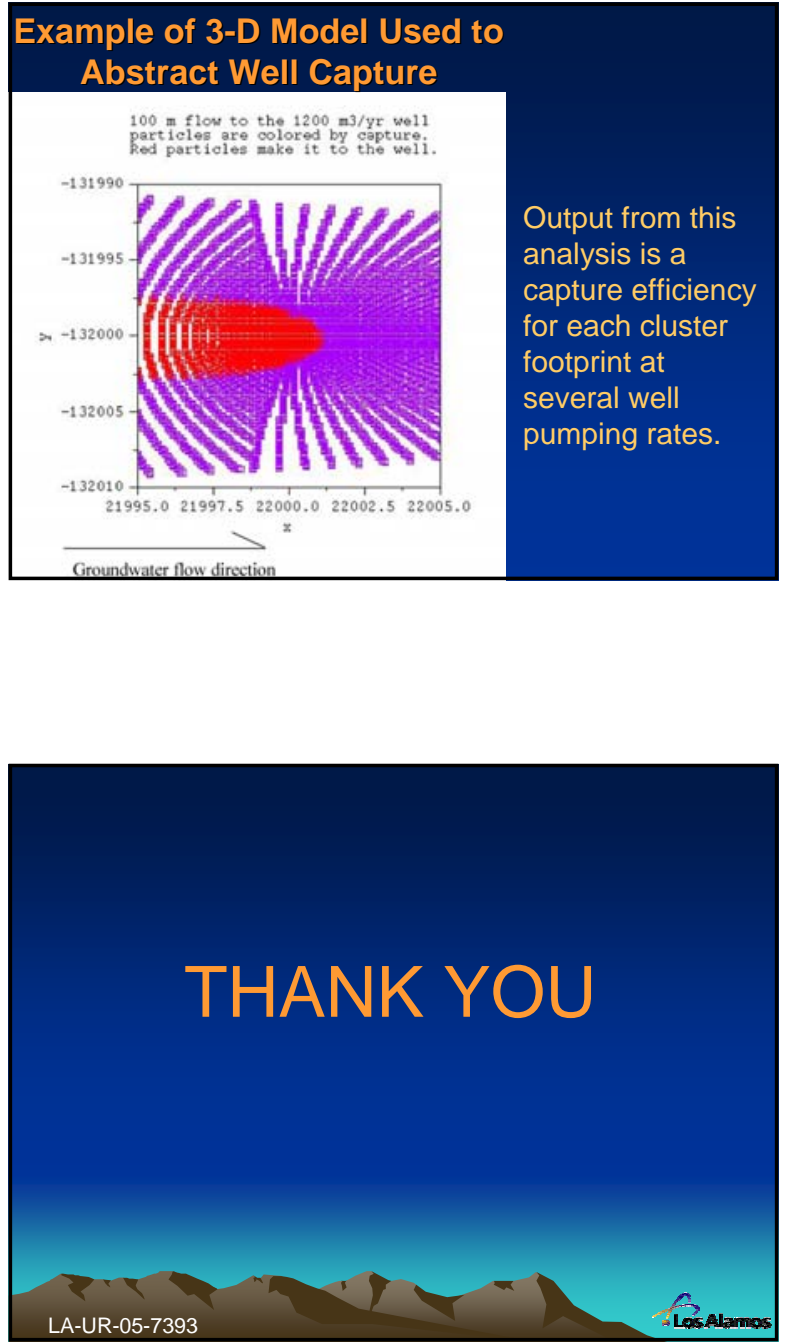

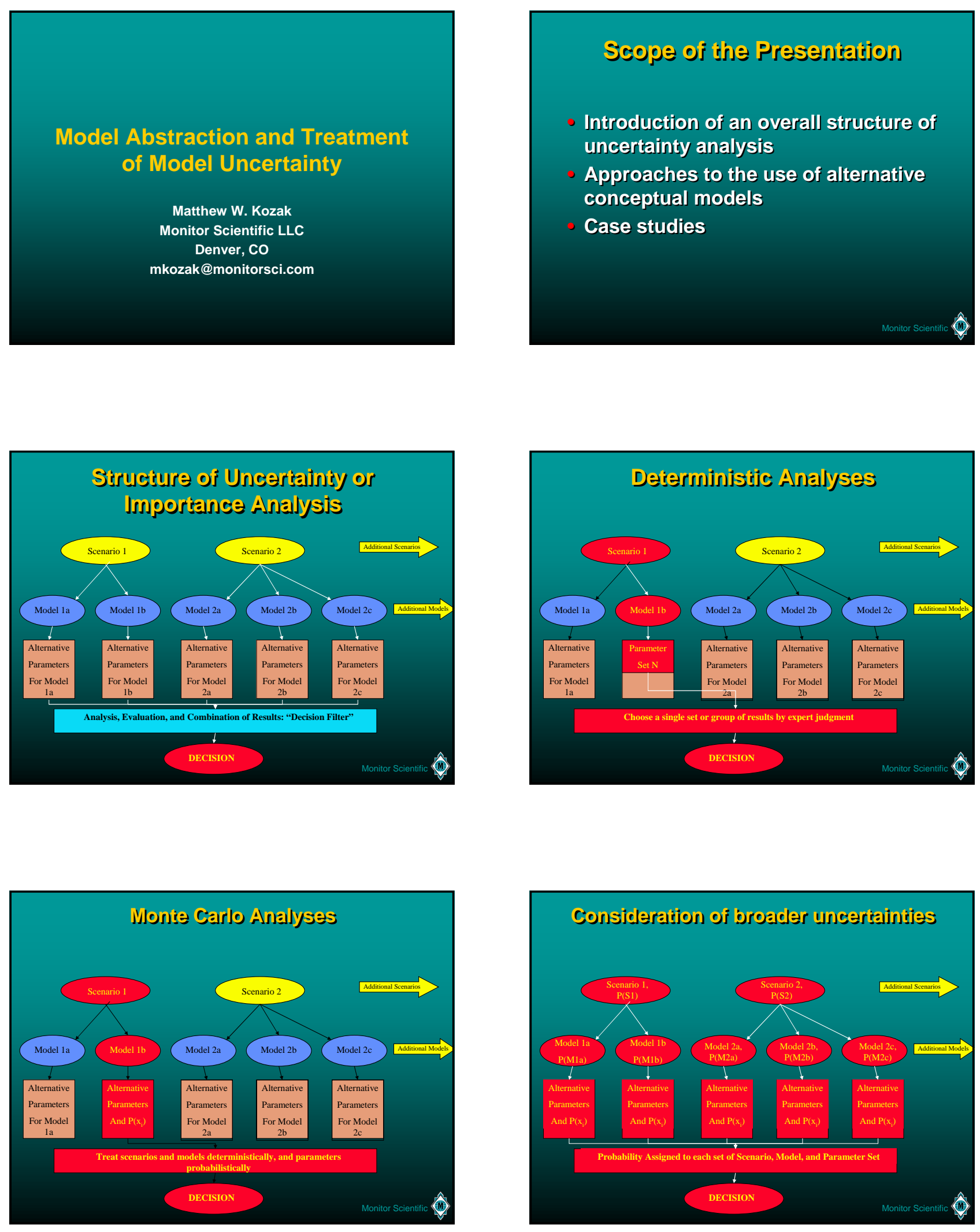


\section{Origins of alternative conceptual models}

Differing assessment context - Degrees of conservatism Regulator vs. developer Differing analysts

Differing scenario definitions Exploratory conceptual models Evaluation of alternative assumptions Performance margin analysis

\section{Perjormance margijn analysis}

Evaluate assumptions that are credible, but difficult to defend A quantification of "conservatism" Can provide strength to licensing arguments
Case study: Yucca Mountain volcanism

Aggregated expert opinion has been used to estimate the probability of igneous activity at the repository is 1.6E-8 $\mathrm{y}^{-1}$

DOE's consequence analysis is carried out in a very conservative manner

The dike is not diverted by thermally-generated stresses The dike is not diverted by themally-generated stresse The vase puckage does not thit reases from the ful No cleanup of ash occurs at the compliance point Wind direction is uniform toward recepto Ash fall of respirable particles

Vents only form at drifts

The repository meets regulatory criteria even with these assumptions

EPRI undertook a performance margin analysis
Case study: Vaalputs, South Affical near field modeling

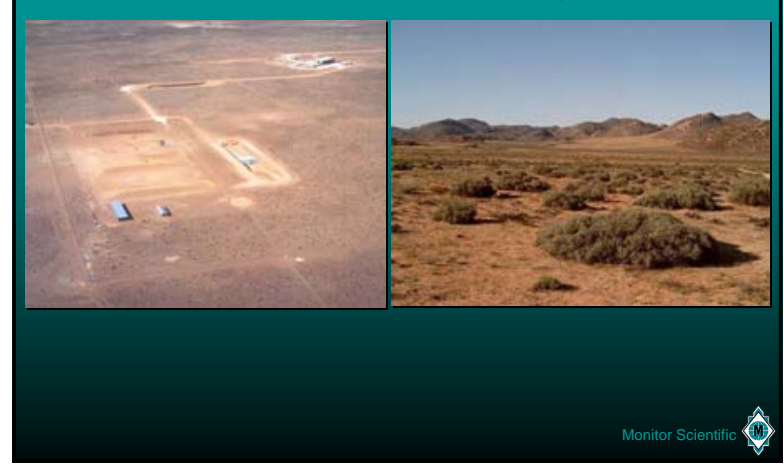

\section{Vaalputs conceptual model}

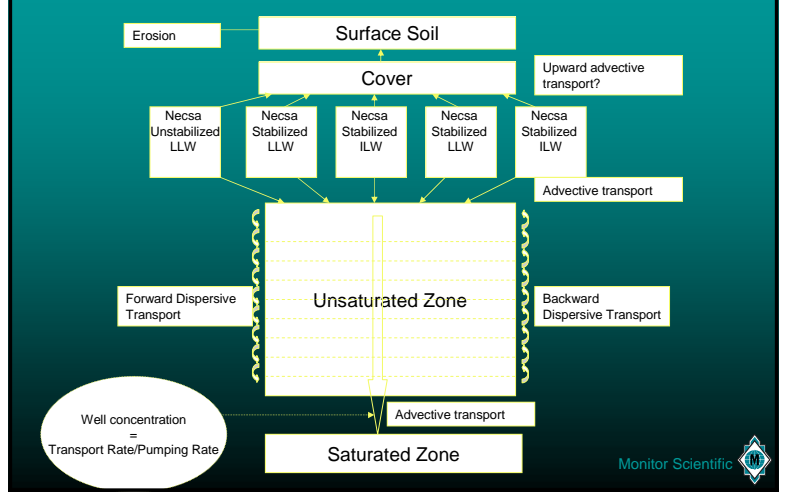




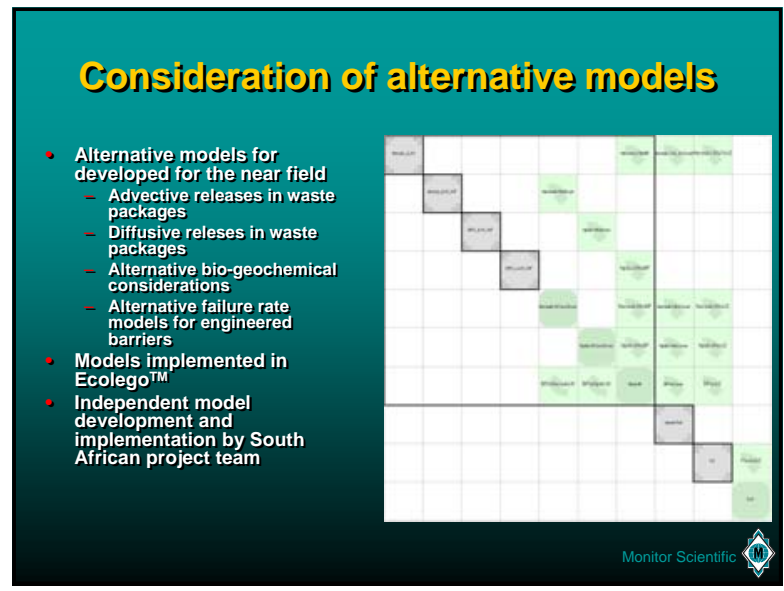

\section{Model Abstraction}

- The process of model abstraction is not just derivation of simple from complex, or more conservative from less

- Viewed as part of a more comprehensive approach to model uncertainty

Model intercomparison seen as a primary tool for producing credibility

Necessarily involves consideration of alternative points of view

\section{Sujmmary}

Full treatment of uncertainty includes more than just Monte Carlo treatment of uncertain parameters

The questions that are answered through treatment of

model uncertainty can be of greater interest to the regulator

than parameter uncertainty

Comparing results of alternative models can

Address technical questions about relevant system behavior

Avaluate conservatism

Evaluate conservatism
Provide quality assurance

Strengthen arguments for the licensing case

Address model abstraction through model intercomparison 
Input Distributions

Model Abstractions

\section{SESSION 1 AND 2} ISSUES AND NEEDS

\section{INPUT DISTRIBUTIONS (COVERED YESTERDAY)}

Distribution forms

Data issues (local; literature; subjective; data deficiencies)

Distribution sampling in probabilistic simulations

Distribution pitfalls/issues

Correlations

Compounded conservatism

Spatial and temporal averaging

Plausibility of sampled values from distributions

Iterative process: distribution development, simulation, sensitivity and uncertainty analysis

Missing uncertainty components

\section{NEEDS AND ISSUES}

Retrospective review of existing PAs

Post-audit review of distributions from model output Credibility of results

Uncertainty of dose conversions and scenario parameters

Input distributions: biotic components

Establishing advantages of probabilistic models

Examples, documentation

Guidance for developing PDFs

Skepticism concerning the value of probability modeling Credibility of simplified probabilistic models

Multiple sites with probabilistic modeling experience

Positive reactions: regulators

Take advantage of the probabilistic modeling experiences of other sites

\section{MODEL ABSTRACTION}

$\times$ Issues

+ Model objective can influence the need for and approach to simplification.

When can the system be simplified?

When is deterministic enough?

+ Advantages of a simplified model Computational efficiency

More accessible to stakeholders

Models informing models

\section{MODEL ABSTRACTION}

$\times$ Quality Assurrance

$\times$ Quick response to Special Analyses

\section{MODEL ABSTRACTION}

$\times$ Needs

+Comparison criteria - link to decision

Structured systematic approach to abstraction

Retrospective reviews of existing PAs

Model intercomparisons

Alternative conceptual models 
WSRC-STI-2008-00333, REVISION 0

SESSION 3 - IMPLEMENTATION OF PROBABILISTIC SENSITIVITY AND UNCERTAINTY ANALYSIS 
WSRC-STI-2008-00333, REVISION 0

This page intentionally left blank. 


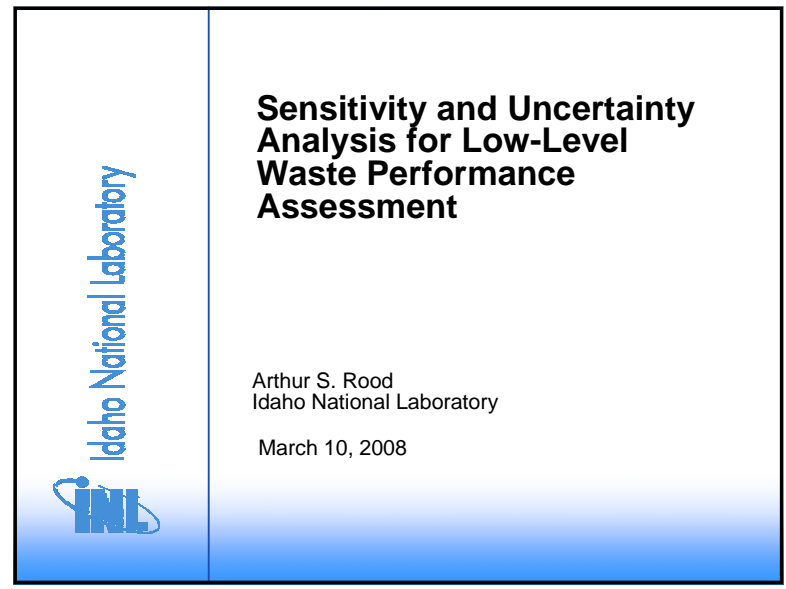

\section{PA Modeling Process}

- Develop conceptual model

- Develop numerical model of flow and transport

- Develop source term - Screen radionuclides

- Model validation/calibration

- Abstract numerical model into a simpler form

- Perform deterministic model runs

- Perform sensitivityluncertainty analysis

- One-Factor at a Time (OFAT) sensitivity analysis

- Parametric uncertainty/sensitivity analysis

\section{Objective}

- Outline a process for performing sensitivity/uncertainty analysis for a lowlevel waste performance assessment

- Illustrate the process using the performance assessment for the Radioactive Waste Management Complex at the Idaho National Laboratory

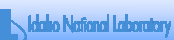

\section{Sensitivity/Uncertainty Analysis Process}

- Define the assessment question

- Use the deterministic results to select important radionuclides

- Perform OFAT sensitivity analysis

- Select parameters to consider stochastically and develop distributions

- Develop tools to calculate parametric uncertainty

- Perform parametric uncertainty analysis

- Perform sensitivity analysis using the results of the parametric uncertainty analysis

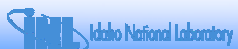

\section{Define the Assessment Question}

- What is the all-pathway EDE in a given year after closure to a hypothetical person who resides $100 \mathrm{~m}$ downgradient from the disposal facility?

- Does the dose exceed the performance criteria?

- Physical characteristics and ingestion rates are specified by the exposure scenario and not considered stochastically

- Uncertainty not considered for dose coefficients or food chain transfer factors

- The assessment question is deterministic in nature

- Uncertainty in model-predicted doses arise from uncertainty in predicting radionuclide concentrations in environmental media

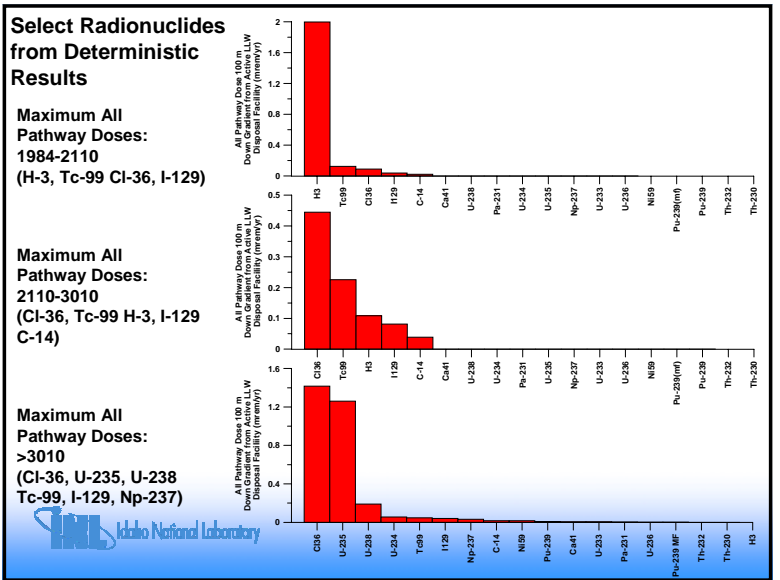




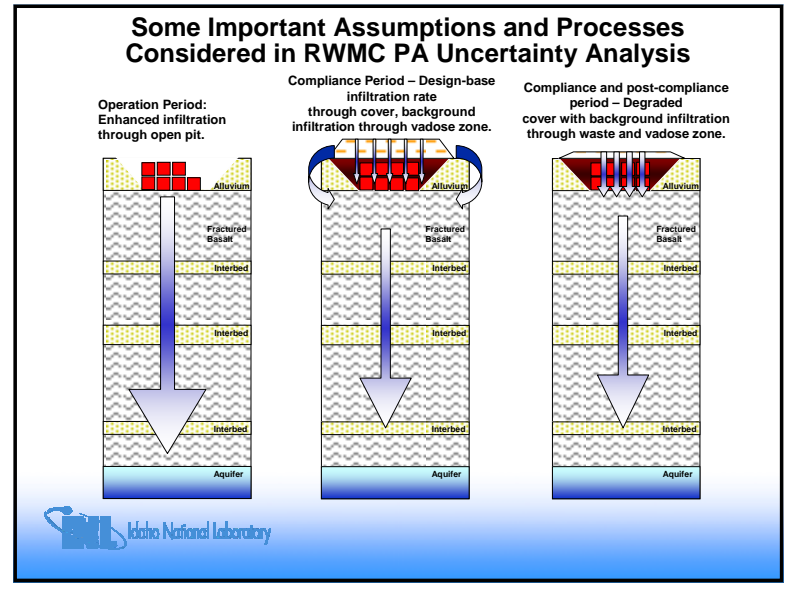

Factors Considered in OFAT Sensitivity Analysis in the RWMC PA

- Delay time in the release of ${ }^{3} \mathrm{H}$ from waste forms

- Installation of infiltration-reducing cover

- Presence of low-permeability zone in aquifer beneath the Radioactive Waste Management Complex

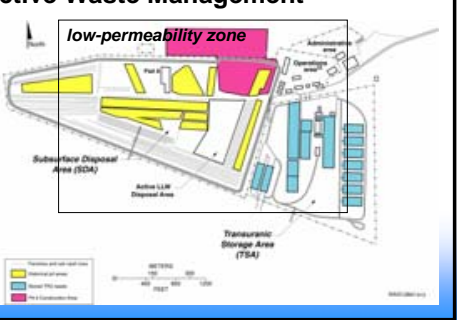

\section{Sensitivity of All Pathway Dose* to} Presence of Low Permeability Zone

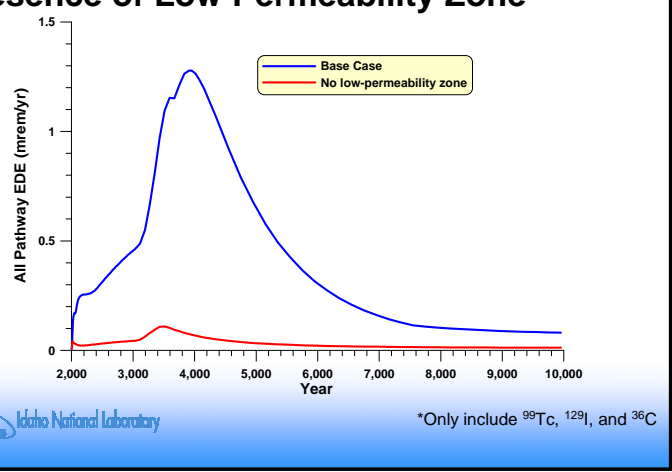

\section{One-Factor-at-a-Time Sensitivity \\ Analysis}

- Illustrates the impact of changing one parameter or assumption on the model results

- Is useful for examining the impact of key assumptions and parameters on model output

- Provides an alternative deterministic answer to the assessment question

\section{Sensitivity of All Pathway Dose to Cover Installation}

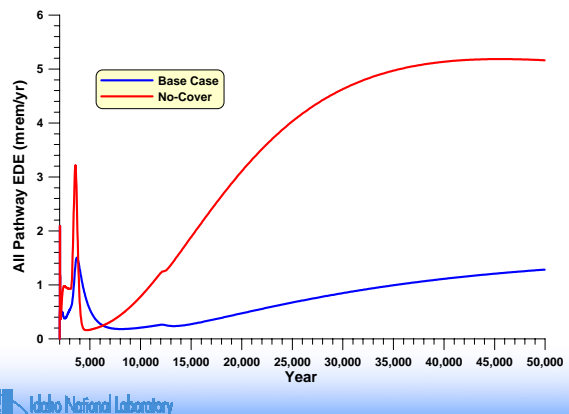

Parametric Uncertainty and Sensitivity Analysis

- Uncertainty Analysis provides a measure akin to the Precision of a model's output given lack of knowledge in the model's input parameters

- Does not address the accuracy of the model which is only addressed through model validation

- Sensitivity analysis provides a measure of the importance of a given parameter in terms of its effect on the variability of the output variable 


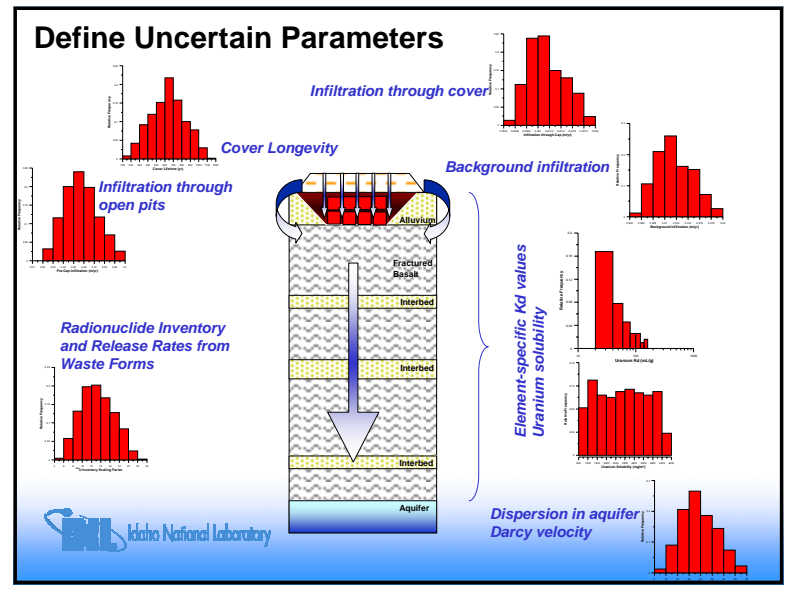

\section{Implementing Monte Carlo Uncertainty Analysis}

- Use models with built-in Monte Carlo drivers

- GoldSim, MEPAS

- "Wrap" models in a Monte Carlo shell

- The "Wrap" is best performed using scripting language like Perl

- The "Wrap" approach offers the most flexibility but requires custom code development to implement a site-specific analysis

- One-hundred model realizations can provide a good estimate of the central value, but more are needed to define the tails of the distribution

- 250-500 realizations combined with tolerance intervals ${ }^{a}$ can provide reliable estimates of the tails of the output distribution

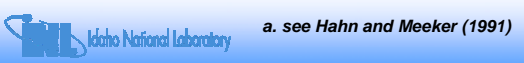

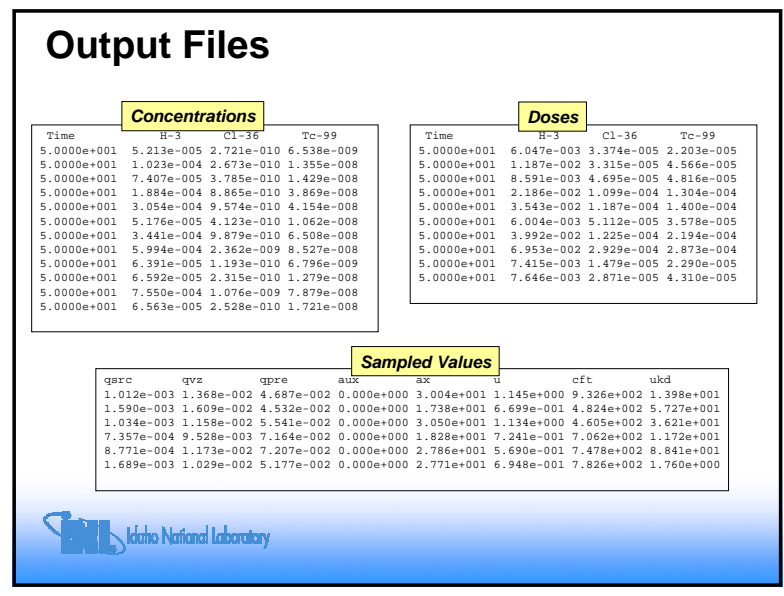

\section{Defining Parameter Distributions}

- The parameter distribution quantitatively expresses the present state of knowledge about the appropriate parameter value

- Parameter distributions may be based on empirical data, but ultimately involve the subjective judgment of the analyst

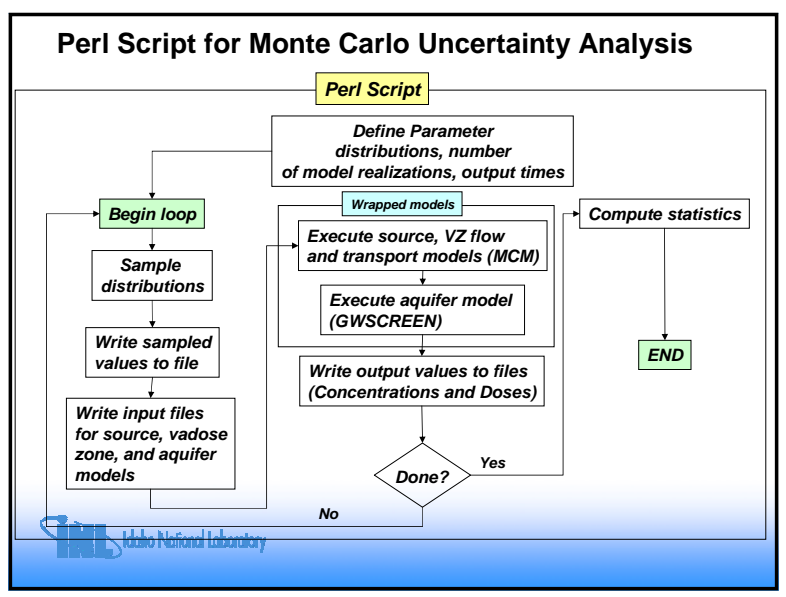

\section{Summary Statistics}

- In many cases, the output distribution of the assessment question spans several orders of magnitude and is not defined by a known

distribution (e.g., normal, lognormal)

- Output can be summarized in terms of percentiles of the output distribution with non-parametric tolerance intervals as described in Hahn and Meeker (1991)

- Plot the output distribution as a function of time 
Temporal Dependence of the Uncertainty in the All

Pathway EDE for the RWMC Performance Assessment

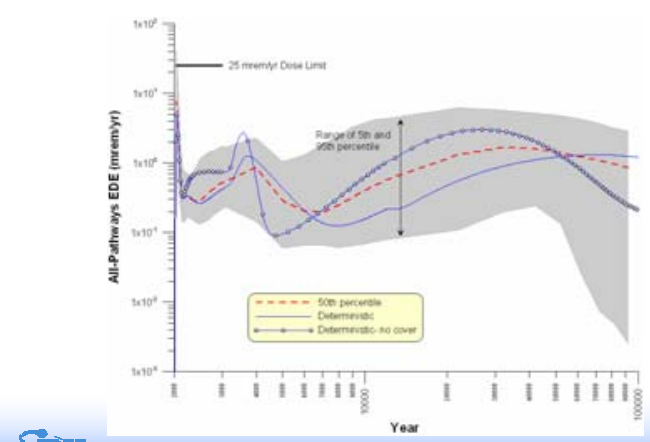

Watha Nofind laberutery

\section{Sensitivity Analysis Procedure}

- Perform rank correlation of output variable to each parameter value

- Rank correlation is non-parametric - does not depend on the distribution of the parameter or the output variable

- Check for significance of the rank correlation coefficient

- Generate scatter plots of output variable and selected parameters

- Approximate the percent contribution to variance

$$
\% V_{i}=\frac{A B S(R C C)_{i}}{\sum_{i=1}^{n} A B S(R C C)_{i}} \times 100
$$
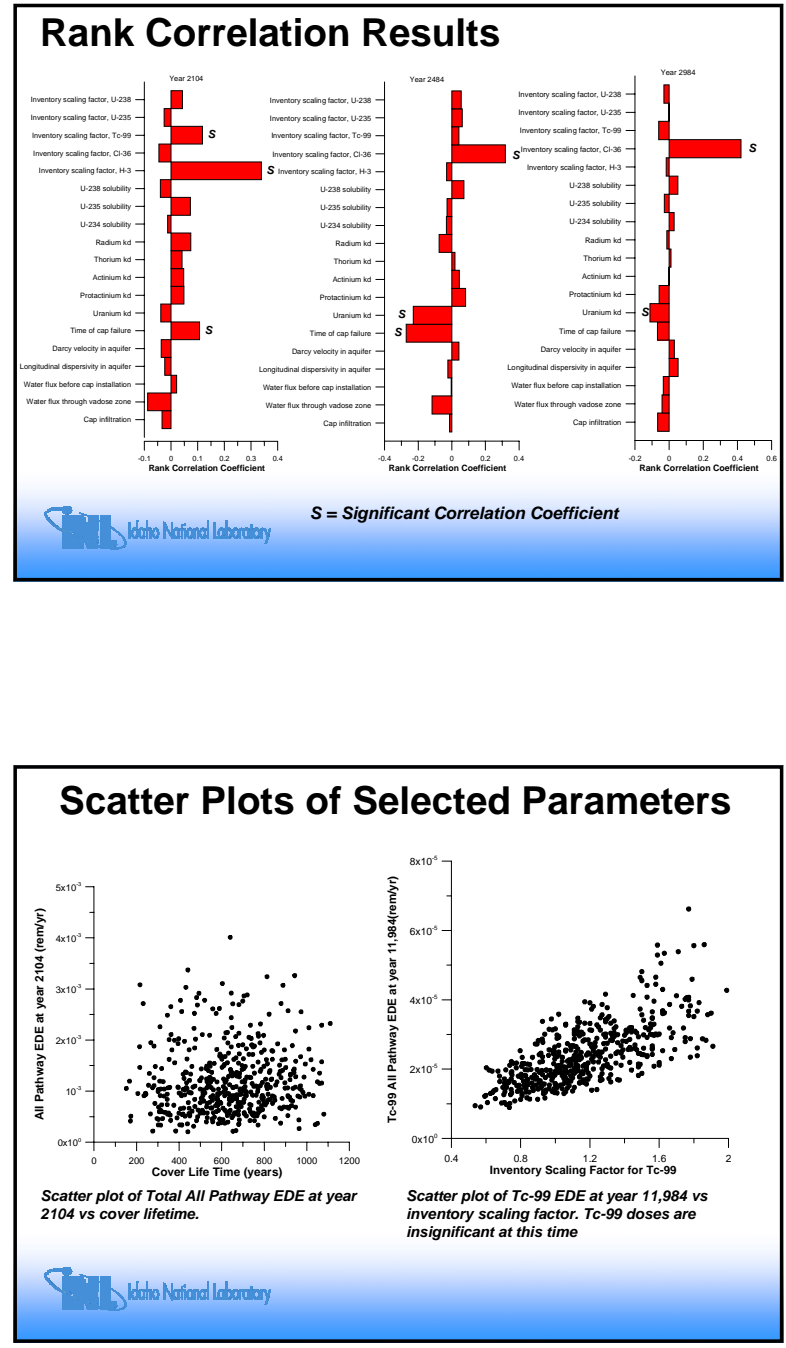
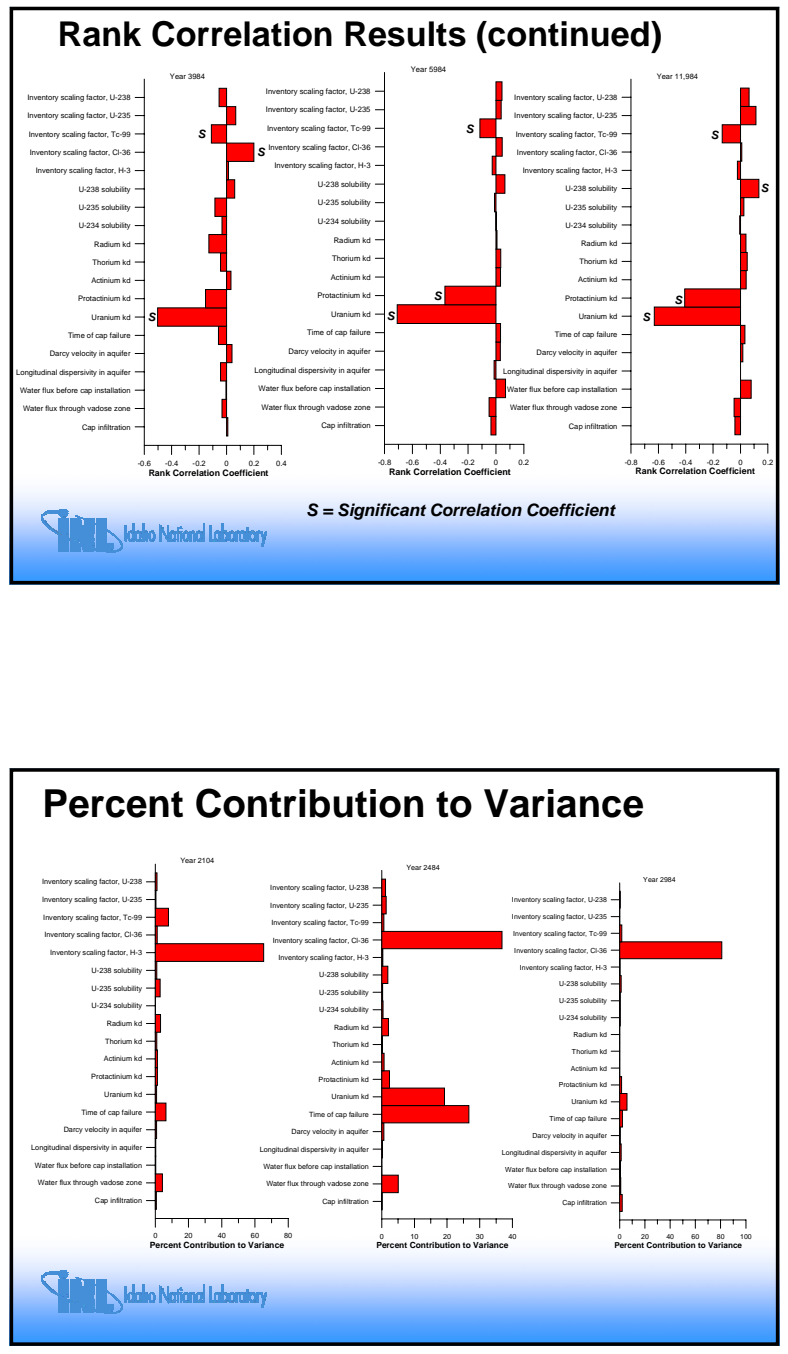


\section{Percent Contribution to Variance (continued)}

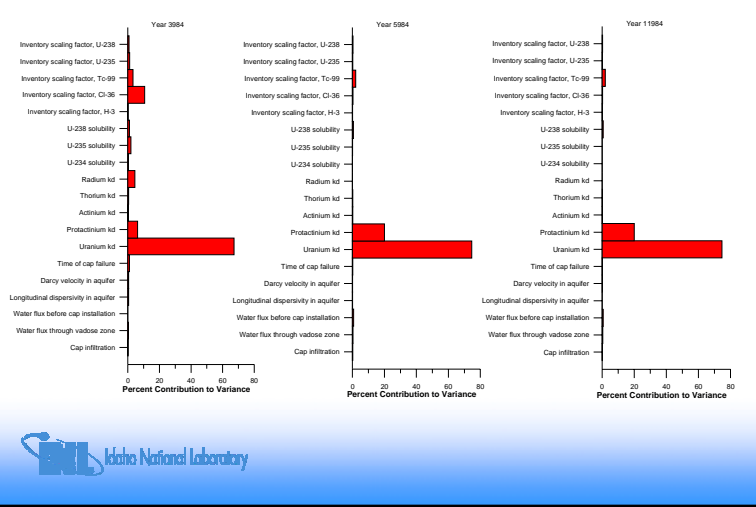

\section{Summary and Conclusions}

- Parameter uncertainty and sensitivity analysis provide a measure of the variability of the model output to choice of parameter values and assumptions

- Parameter uncertainty and sensitivity depend on the output time, output variable, and parameter distributions

- Can provide a measure of the reliability of the model in terms of demonstrating performance objectives

- Does not provide information regarding the accuracy of the model in terms of predicting concentrations in the environment - Only model validation can provide that

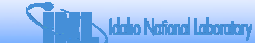




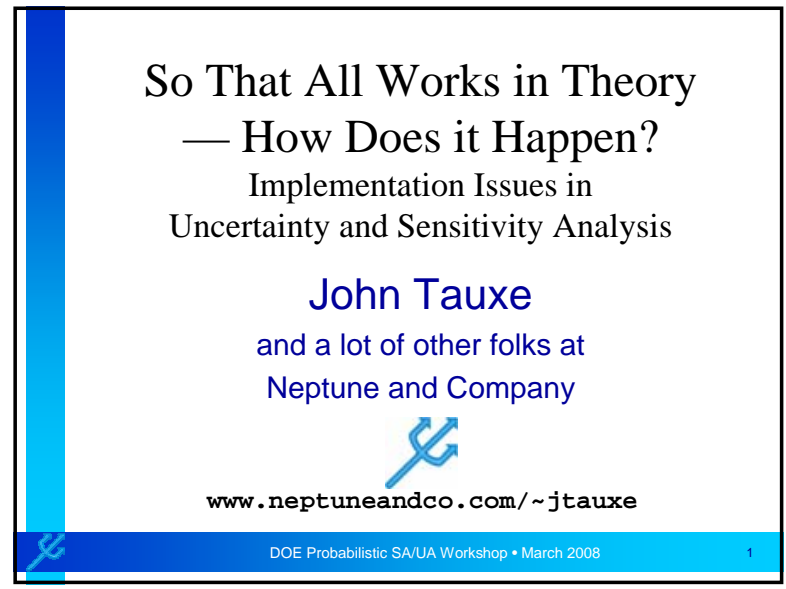

\section{Uncertainty and Sensitivity}

- Uncertainty

All model inputs (and the model itself) are uncertain to varying degrees. This uncertainty is propagated through to uncertain results.

- Sensitivity

Each of these input parameters helps determine the outcome of the model. Some are more influential than others.

SA determines which parameters have the most influence on a particular result (e.g. dose).

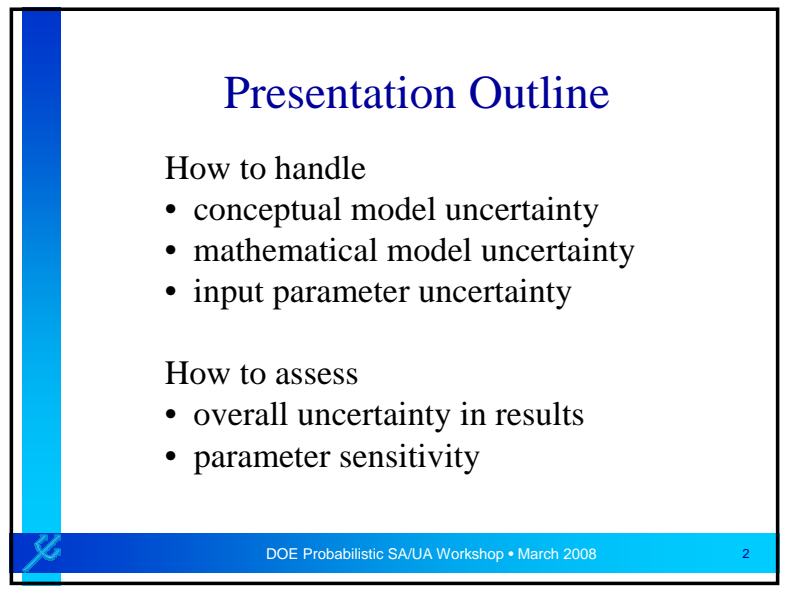

\begin{tabular}{|c|}
\hline Uncertainty in the \\
Conceptual Model \\
Examples of typical CM uncertainty: \\
- hydrogeology and geochemistry \\
- receptor exposure scenarios \\
• engineered barrier failure scenarios \\
- natural evolution of the site \\
These are often handled by including \\
various alternatives in the modeling. \\
DoE Probabisisic sava worksop - marc 2008 \\
\hline
\end{tabular}

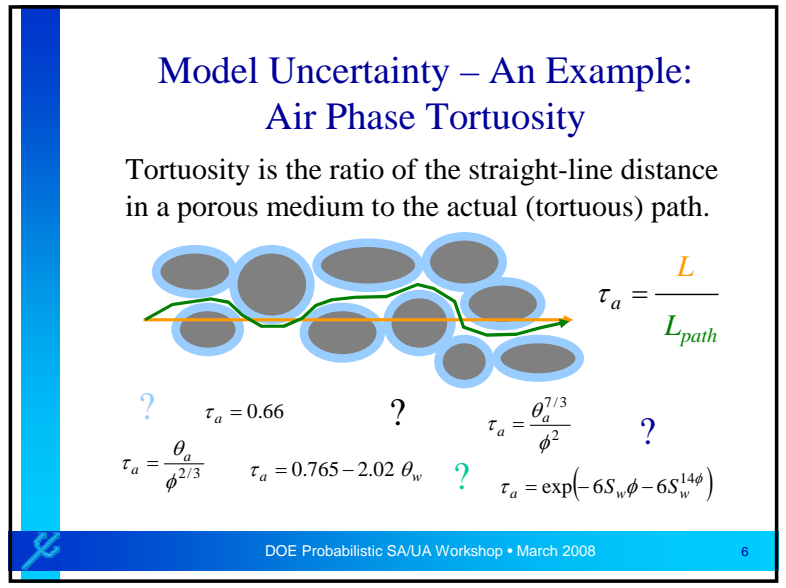



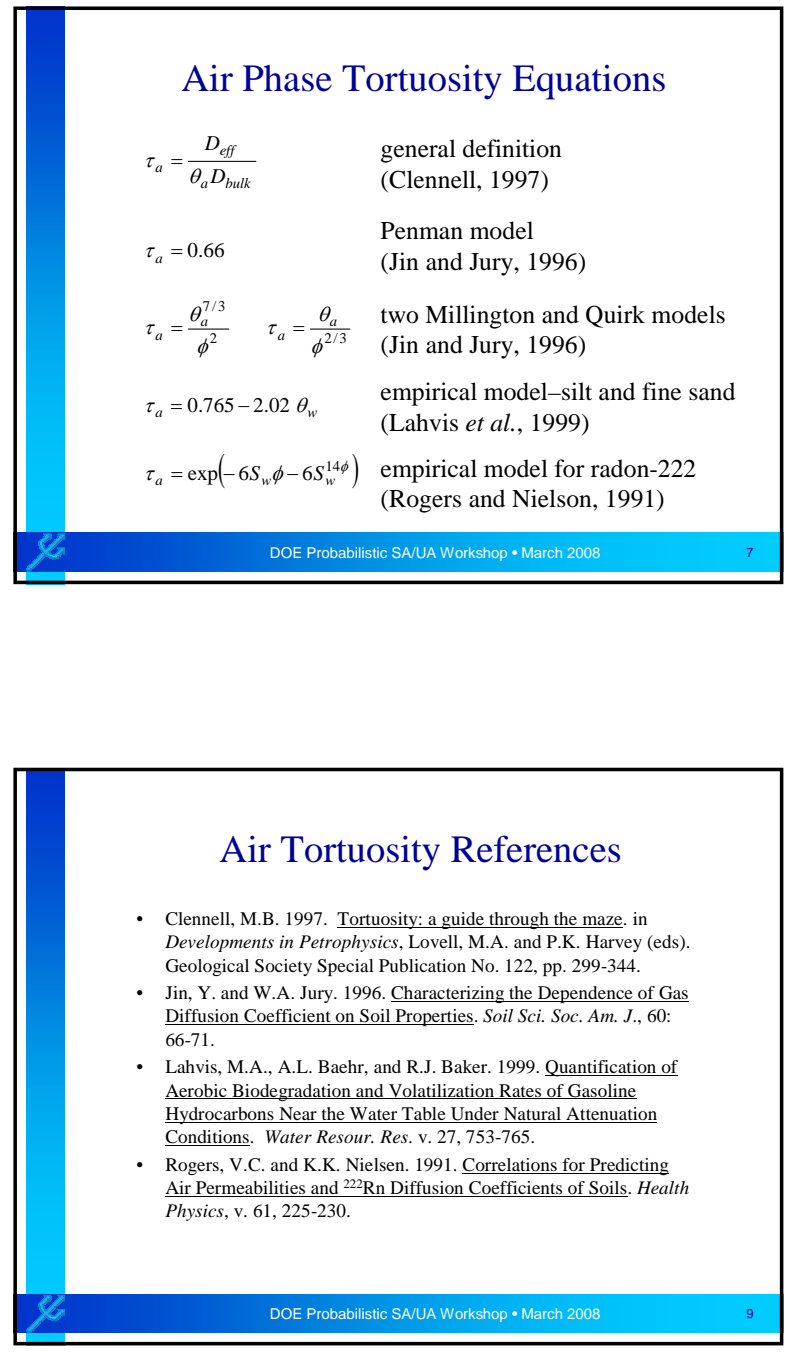

Tortuosity Model Uncertainty: Implementation 2/2

Then use a Selector to

define the actual function.

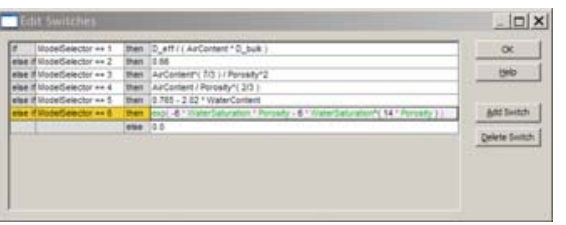

DOE Probabilistic SAlUA Workshop • March 2008
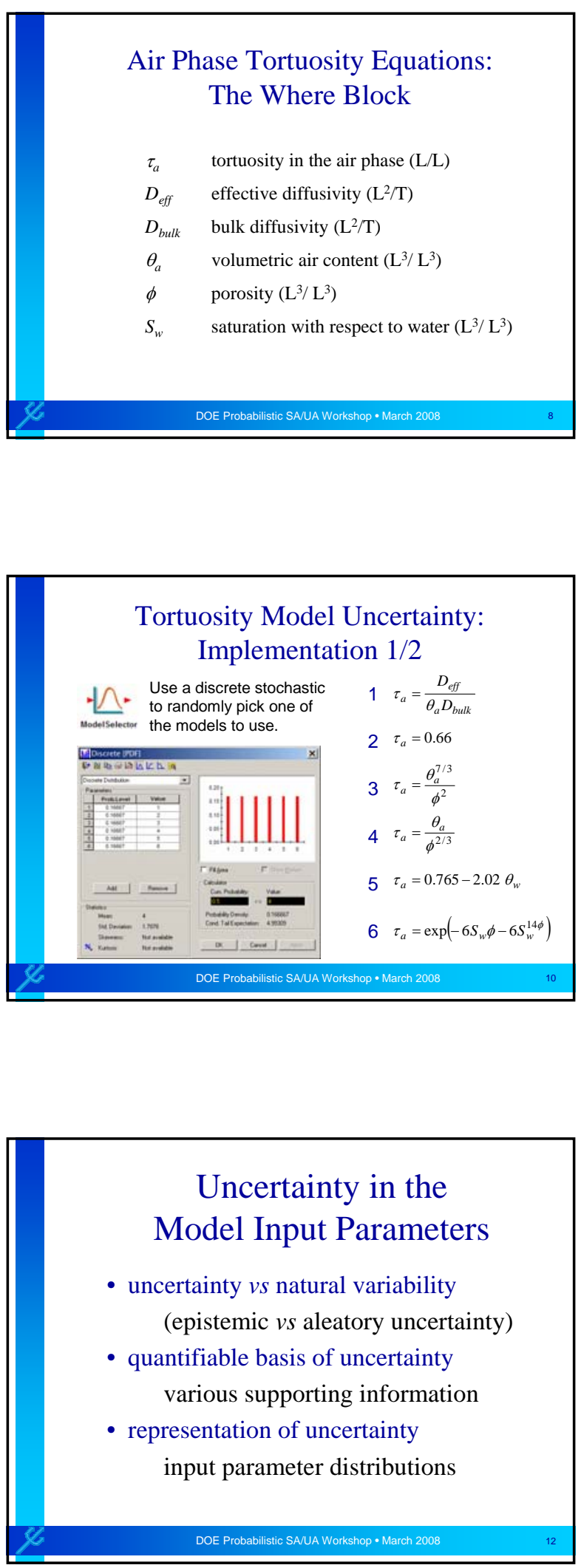

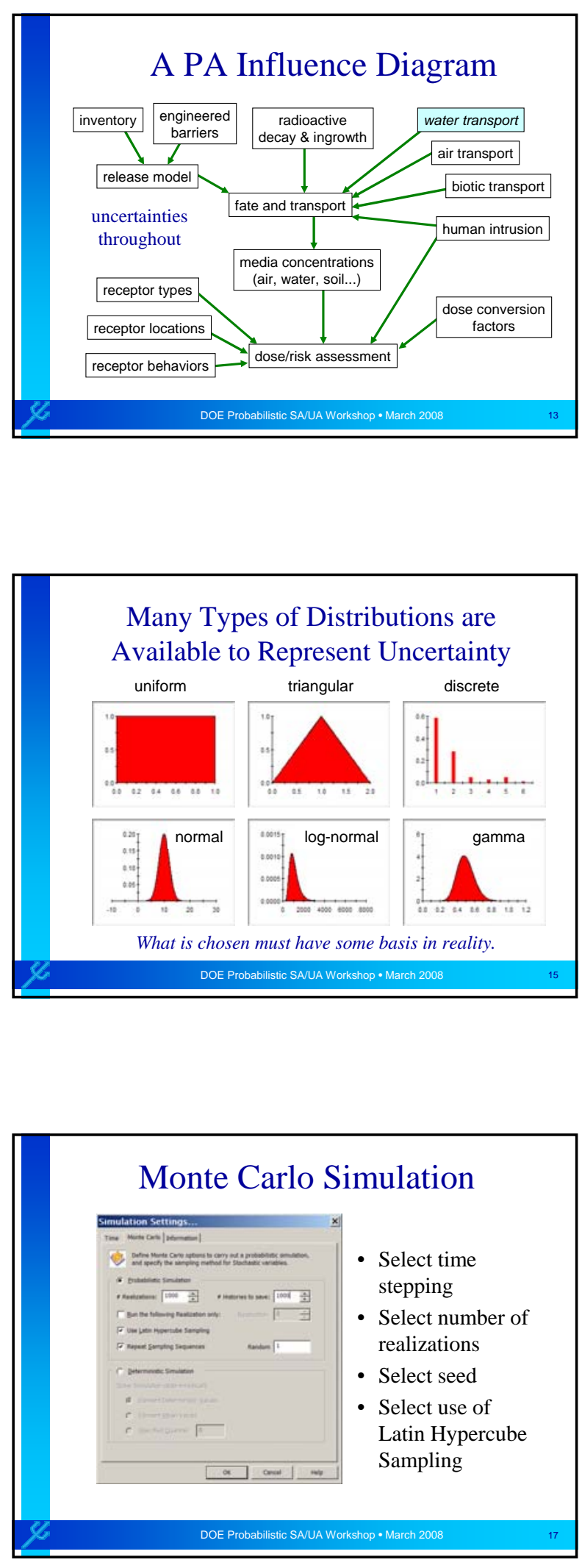
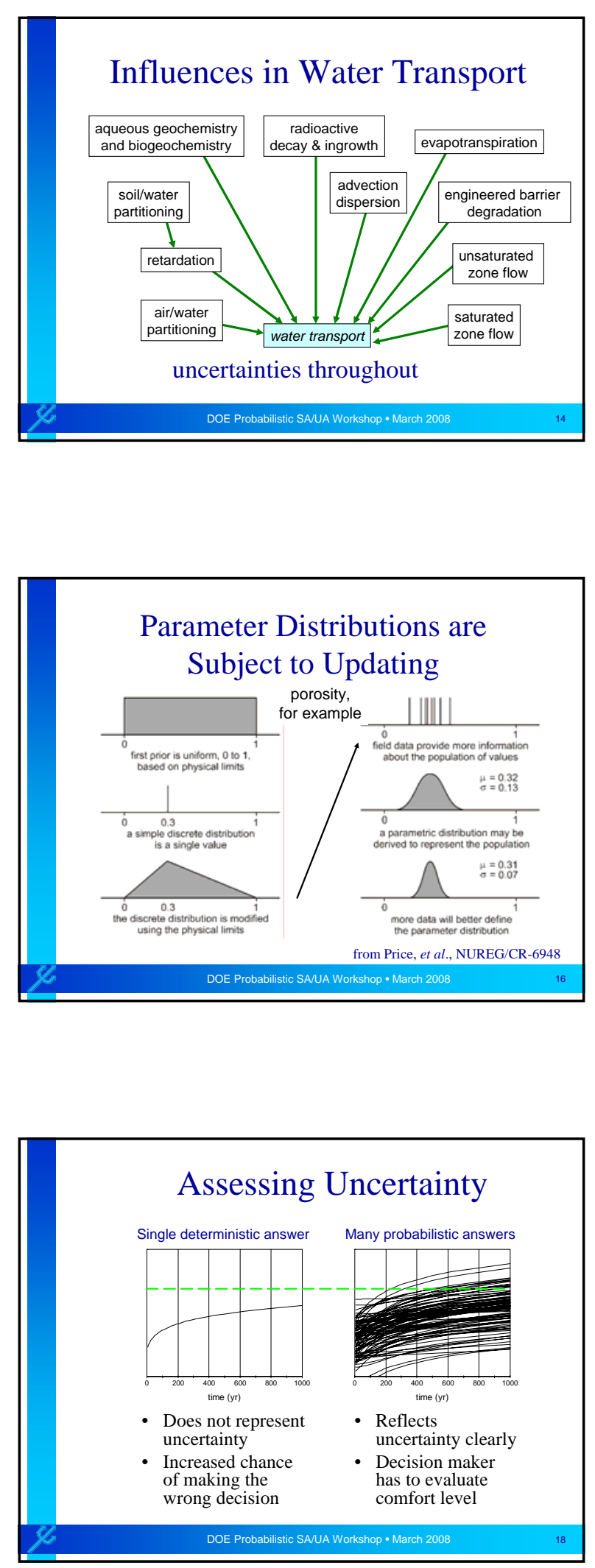

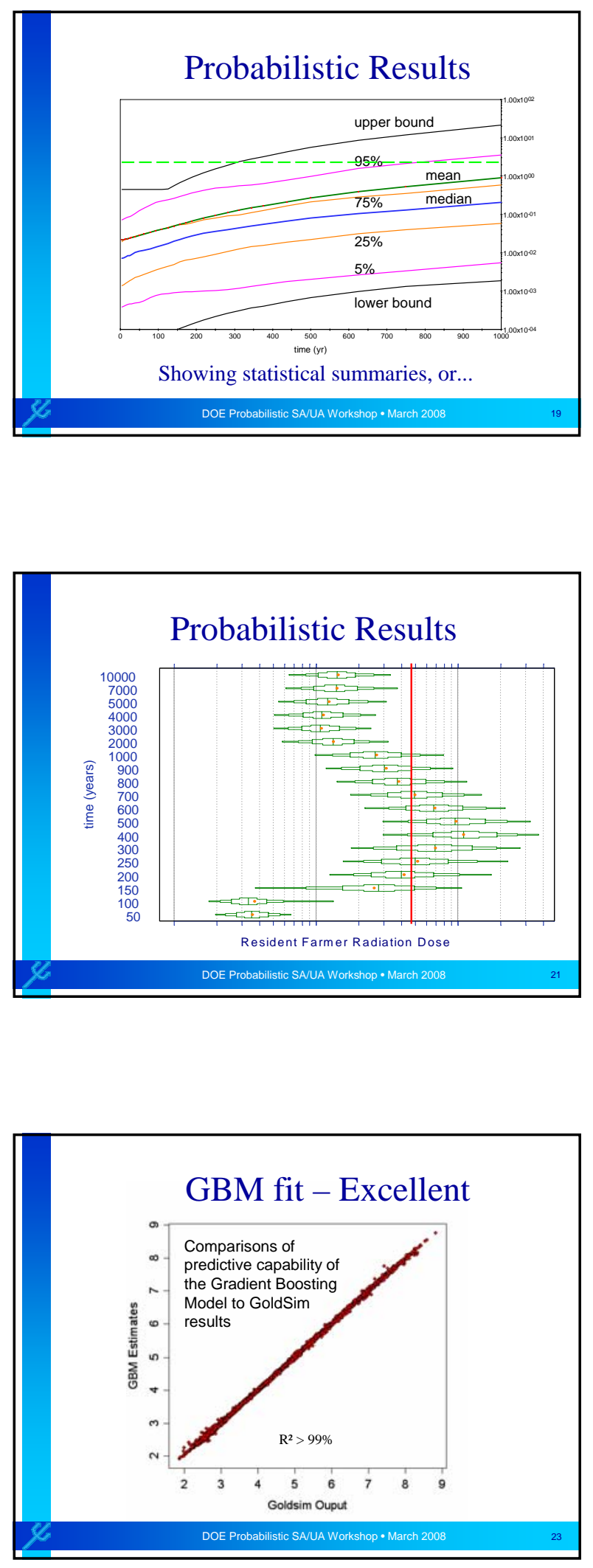

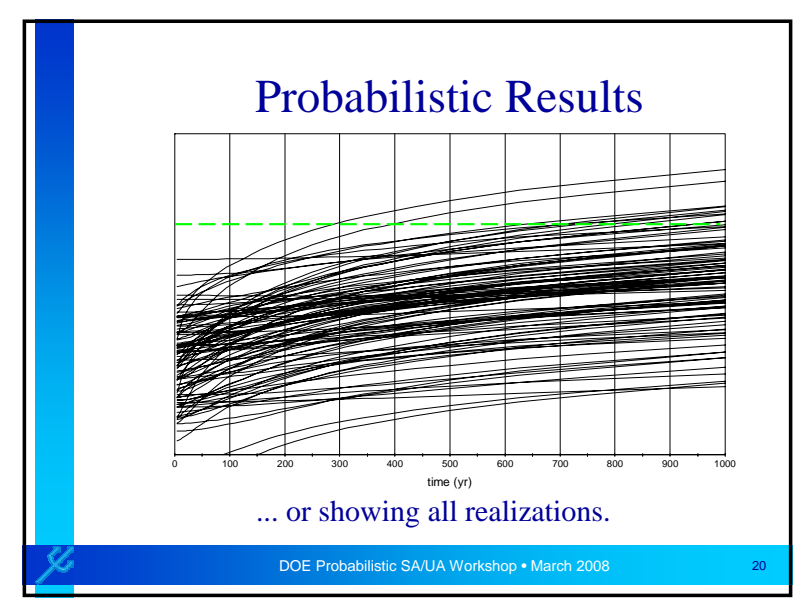

\section{Sensitivity Analysis}

- Based on thousands of plausible PA model realizations.

- A result endpoint is selected, such as a concentration or a future dose.

- SA software, such as that developed at Neptune and Company using $\boldsymbol{R}$, evaluates correlations between all stochastic input parameters and the endpoint.

$\boldsymbol{R}$ is an open-source statistical package:

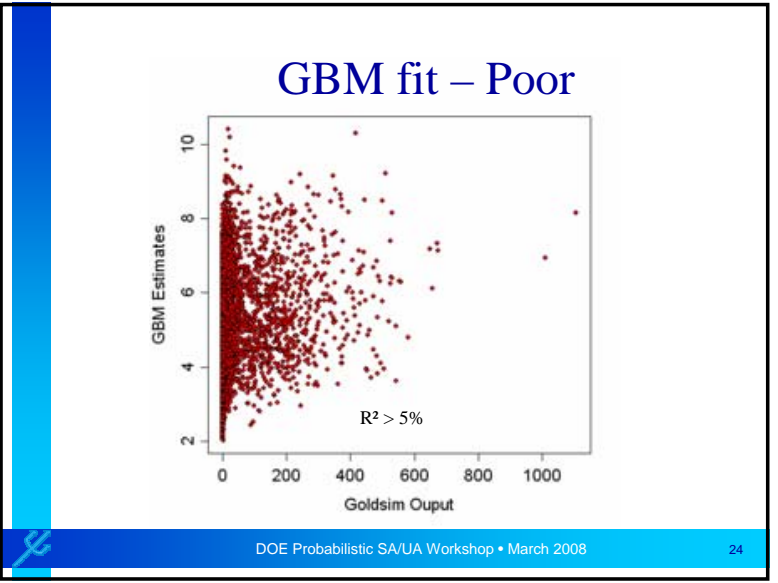



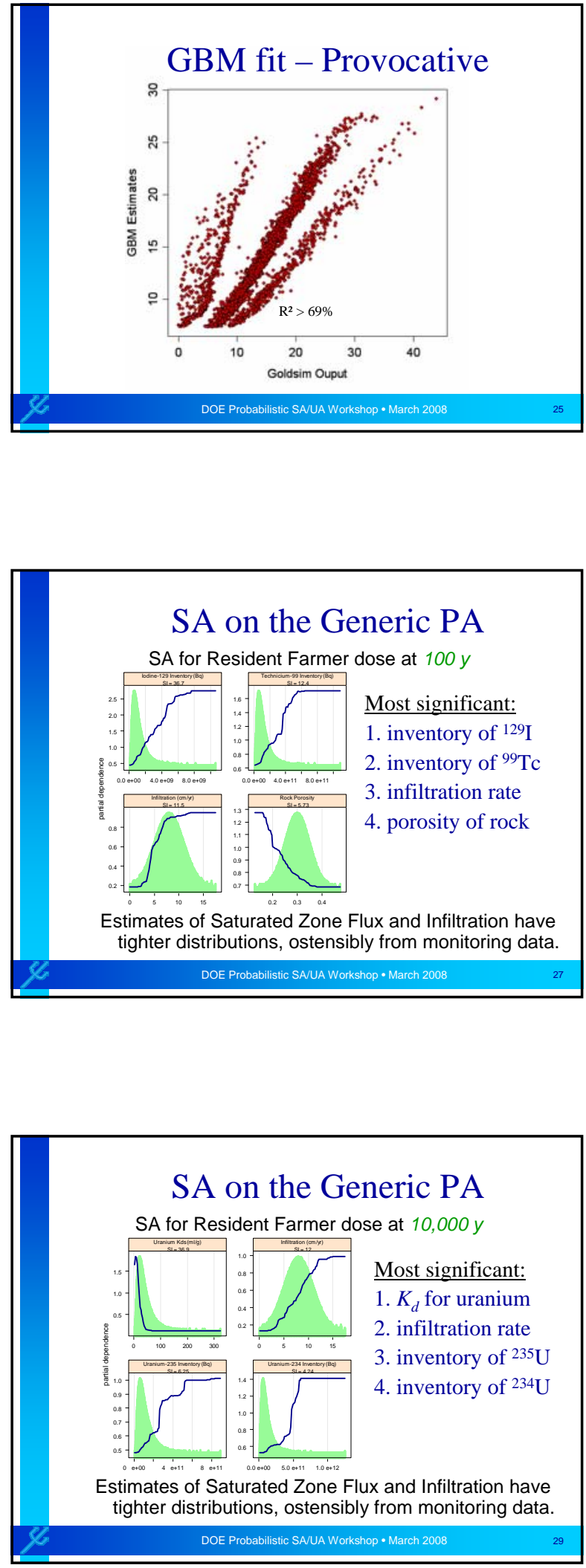
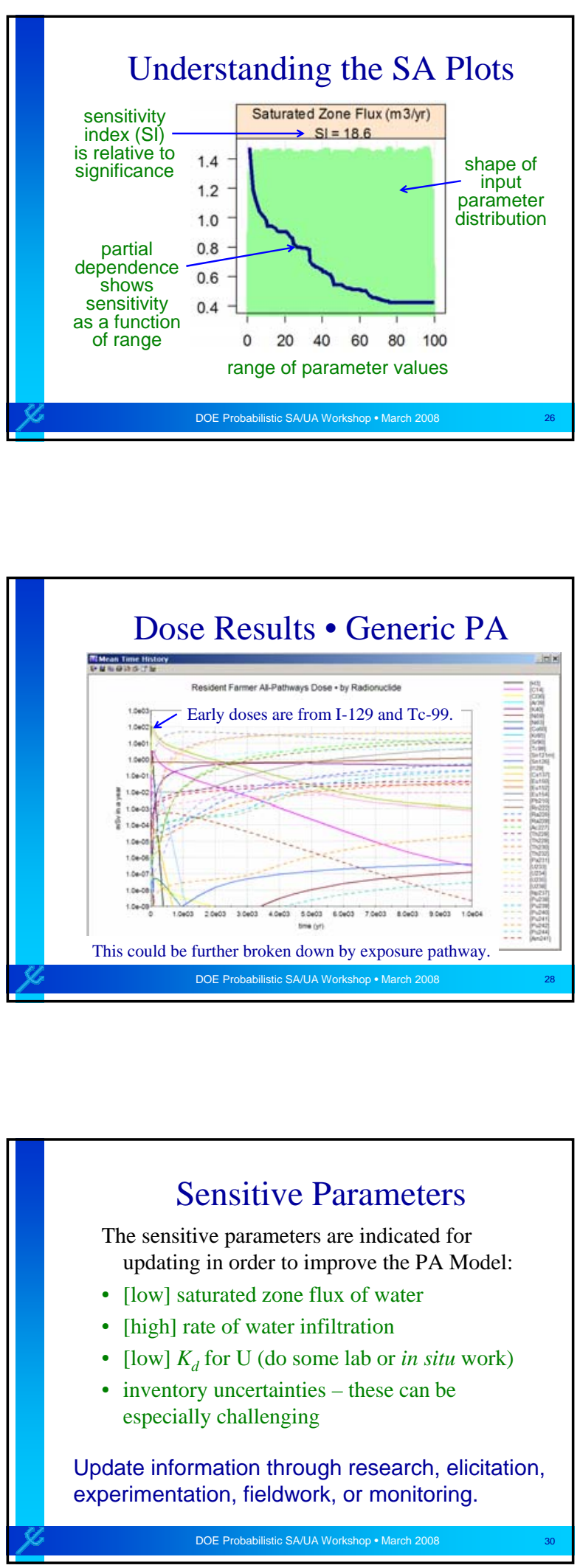


\section{Value of Information Analysis}

- Evaluate VOI from updating activities.

- Determine value of continued updating and refinement (this cannot be done with a deterministic model).

- Decide when updating no longer provides useful information or becomes too expensive (time to stop).

This is essentially a cost/benefit analysis.

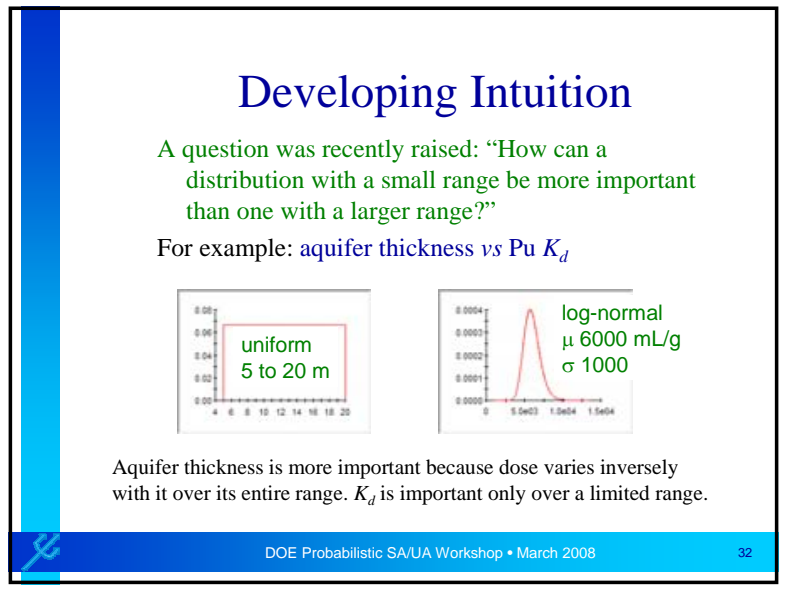

\section{Experiment with the Model!}

The best way to get a feel for your site and how it is represented in a PA model is to fiddle around with it.

Get a copy of the Generic PA model here: http: //WWW . neptuneandco. com/goldsim/generic

\section{Lessons Learned}

- Clarity of model structure makes a difference in how it behaves.

- Appropriate definition of distributions is important.

- Symmetry of even poorly known distributions helps to keep the model statistically well-behaved, aiding the decision-making process.

\section{Take Home Points}

- Performance Assessment is most useful in the context of decision making under uncertainty.

- Sensitivity Analysis can identify the most important parameters at a site, and can direct monitoring or other means of improving defensibility.

- Probabilistic Analysis is necessary to achieve both these benefits.

Regarding Guidance
- NRC is currently leading in this area:
NUREG-1573: PA Methodology
NUREG/CR-6805: Strategy for Hydrogeologic Modeling and UA
NUREG/CR-6948: Groundwater Monitoring Strategy
- DOE G 435.1 could use enhancement,
and would be the most appropriate for
DOE Site PAs
10 CFR 60 and 61 have that seminal
phrase, “reasonable assurance”
DOE Probabilistic SAUA workshop • March 2008




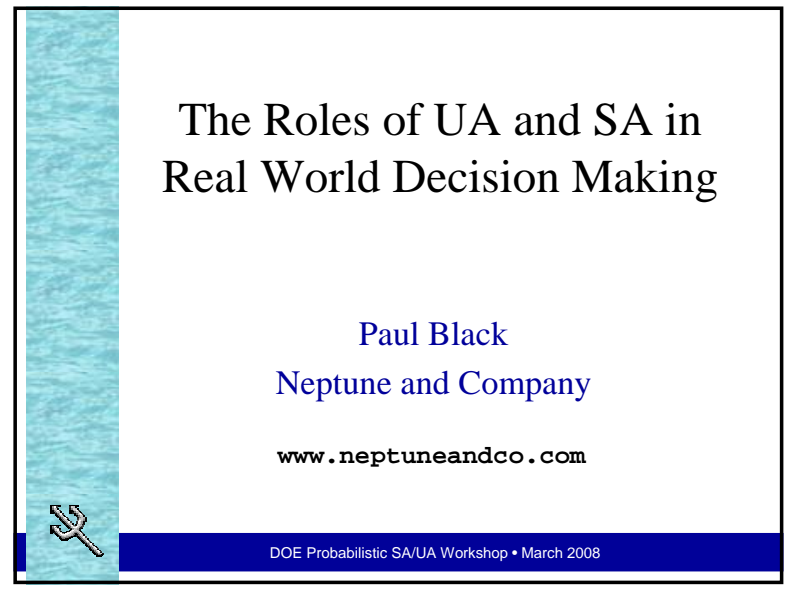

\section{Decision Analysis}

- "Formalized common sense"

- A set of tools for structuring and analyzing complex decision problems

- Logical, reproducible, and defensible decisions in the face of:

- Technical complexity

- Uncertainty

- Costs and value judgments
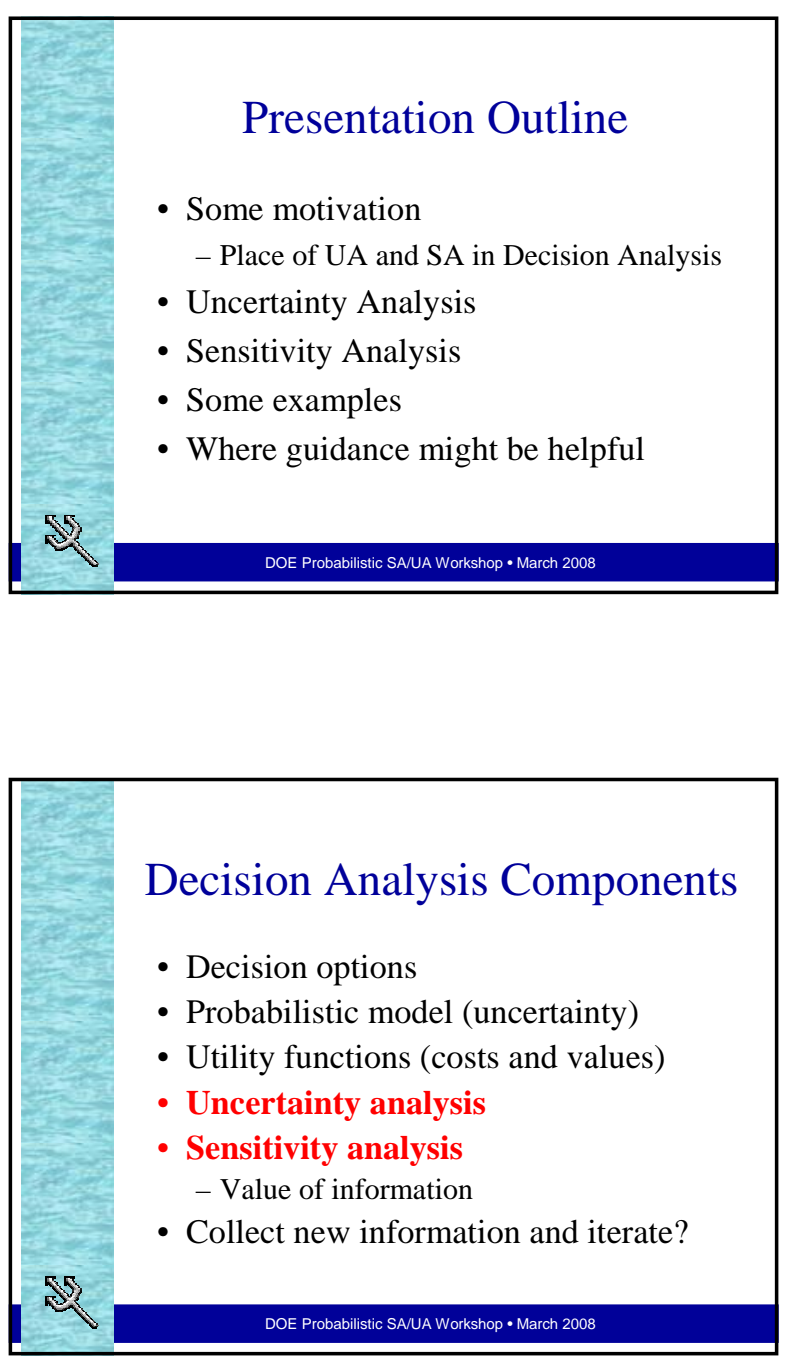

\section{Uncertainty Analysis}

- "The magnitude of the uncertainty of the model responses” (Saltelli)

- Or, the variance or standard deviation component of the output

- How much uncertainty can be tolerated?

- Mean, median, $95^{\text {th }}$ percentile? When comparing output with Objectives

\section{Uncertainty Analysis cont...}

- Saltelli (etc.) assumes uncertainty is characterized

- Meaning, probabilistic modeling

- If deterministic modeling, then UA becomes qualitative only

- Not as easy to defend. 


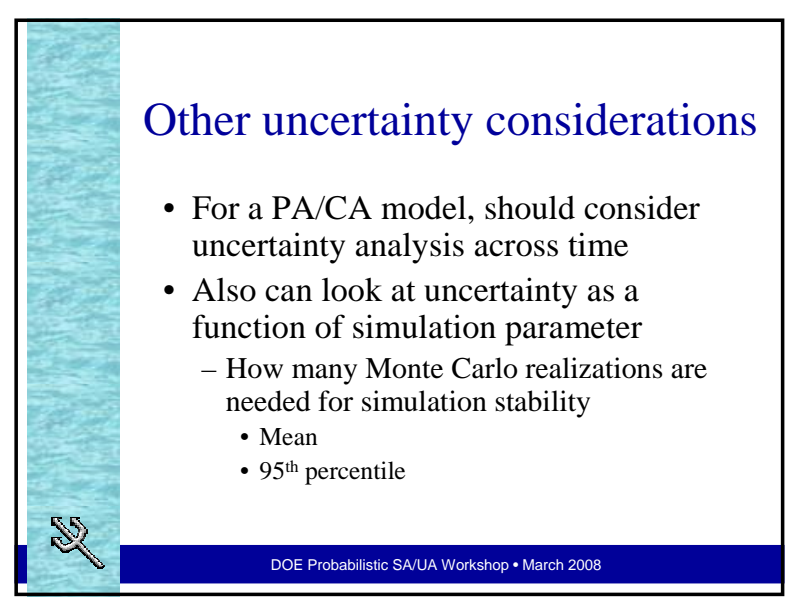

\section{PA/CA Problem}

- How to conduct a sensitivity analysis for a

- Complex,

high dimensional, non-linear,

non-monotonic,

probabilistic environmental model?

DOE Probabilistic SA/UA Workshop • March 2008

\section{Sensitivity Analysis}

- Local sensitivity analysis

- Differential analysis, local to a value of $X_{i}$

$$
\frac{\partial f(\mathbf{X})}{\partial X_{i}}
$$

- One-At-a-Time (OAT)

- Normalize variables to compare sensitivities

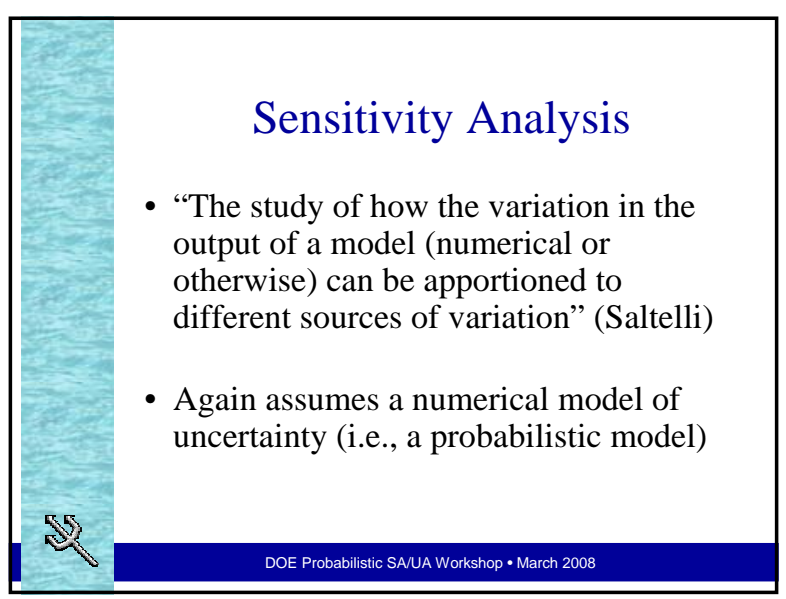

\begin{tabular}{c} 
Sensitivity Analysis \\
Given a model: \\
$Y=f(\mathbf{X})$ \\
$\begin{array}{c}\text { Sensitivity analysis is aimed at } \\
\text { describing the influence of each } \\
\text { input variable } X_{i} \text { on the model } \\
\text { response } Y\end{array}$ \\
\hline
\end{tabular}

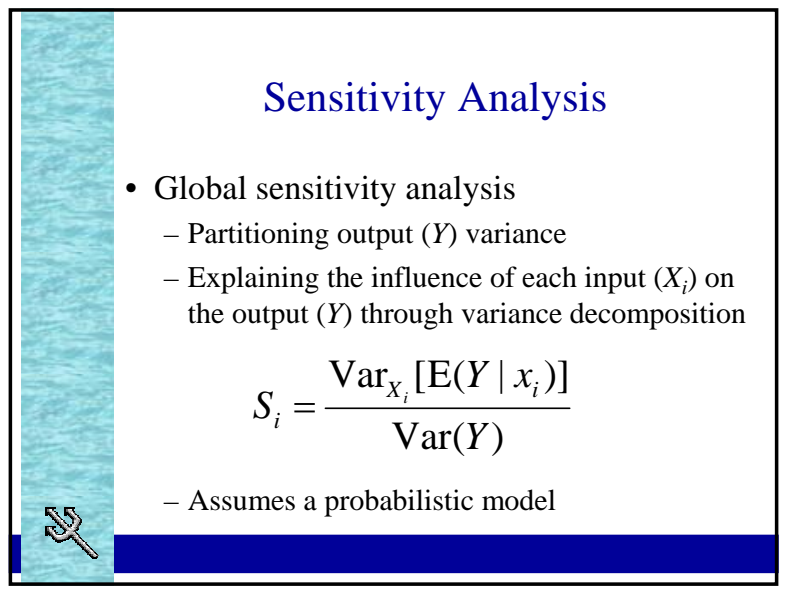



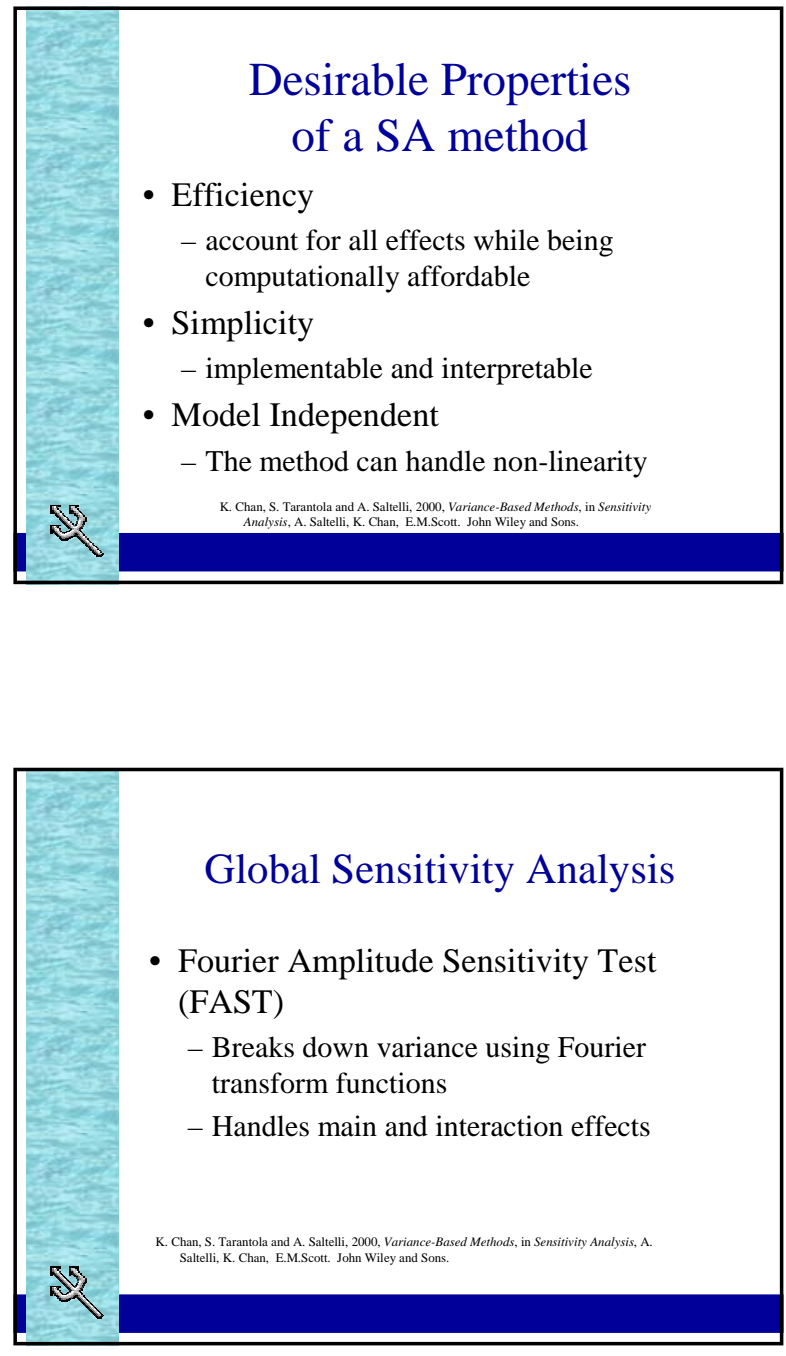

\section{Possible Solutions}

- Data mining or Machine learning methods

- Multivariate Adaptive Regression Splines

- Random Forests (Bagging)

- Gradient Boosting Machine (GBM)

- Multiple additive Regression Trees (MART)

- Basically, fancy regression models for evaluating complex systems or models

- But, they overcome the issues !
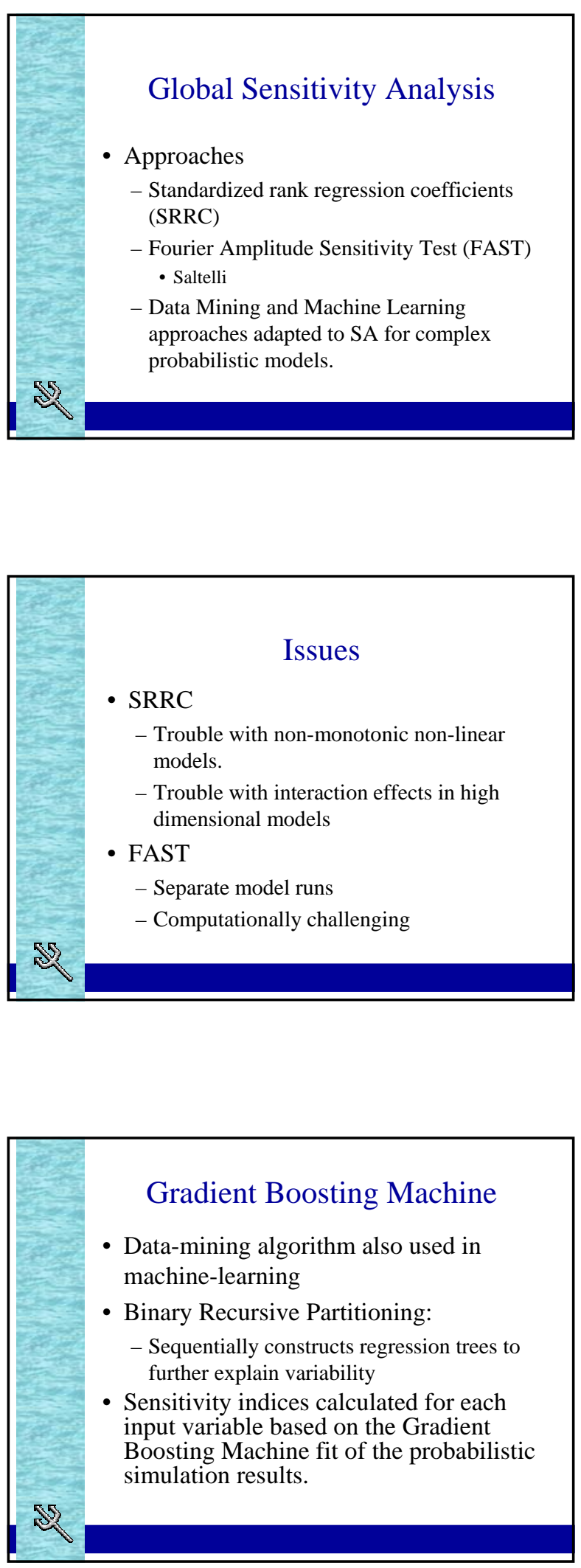


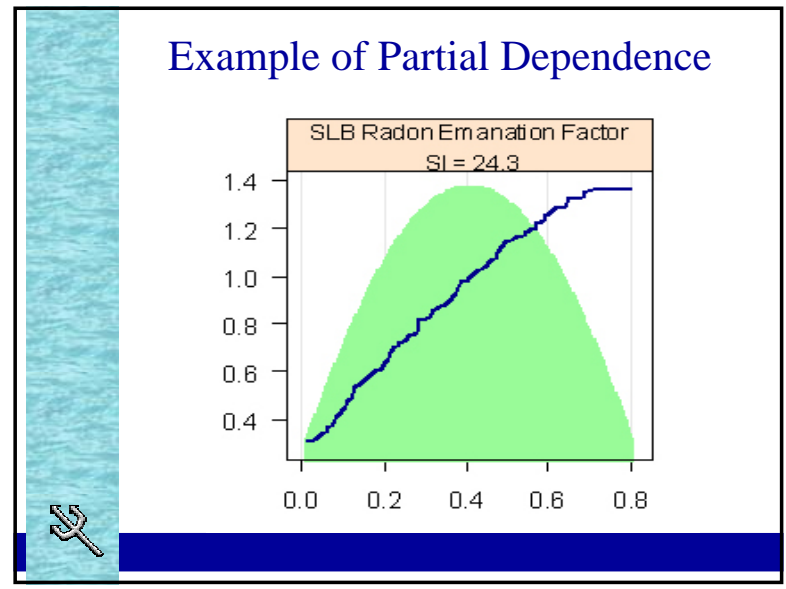

\section{Examples}

- Some from LANL MDA G (2000)

- Overall UA plot

- Overall SA plot

- Some from NTS LLW sites (2006)

- Partial dependence plots for input variables

- Reduction in uncertainty and reduction in conservatism plots

- Simulation uncertainty

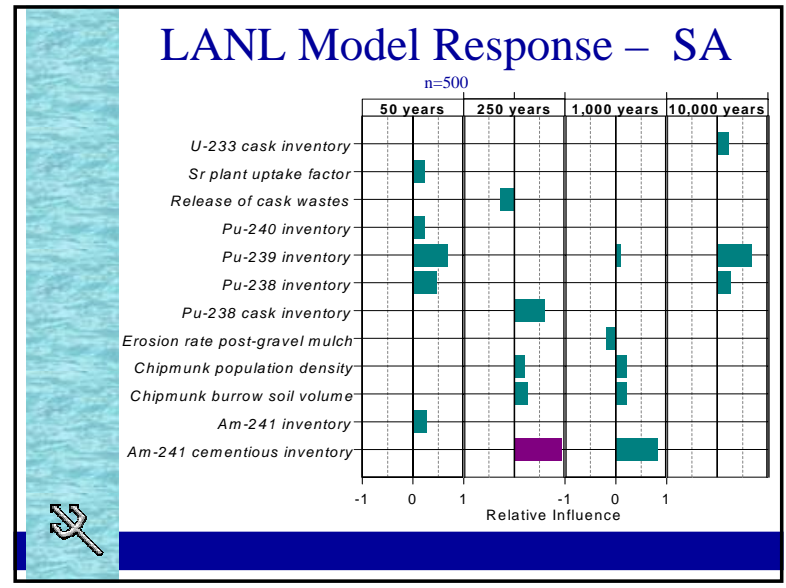

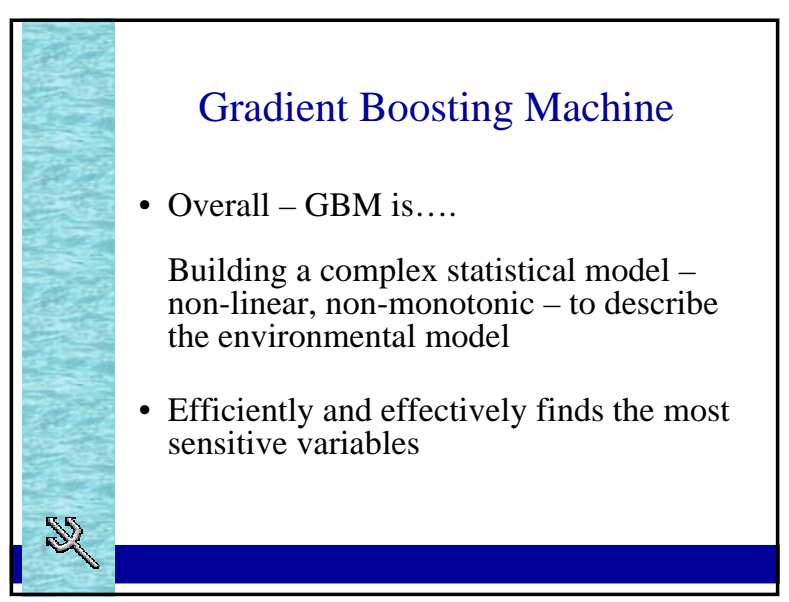
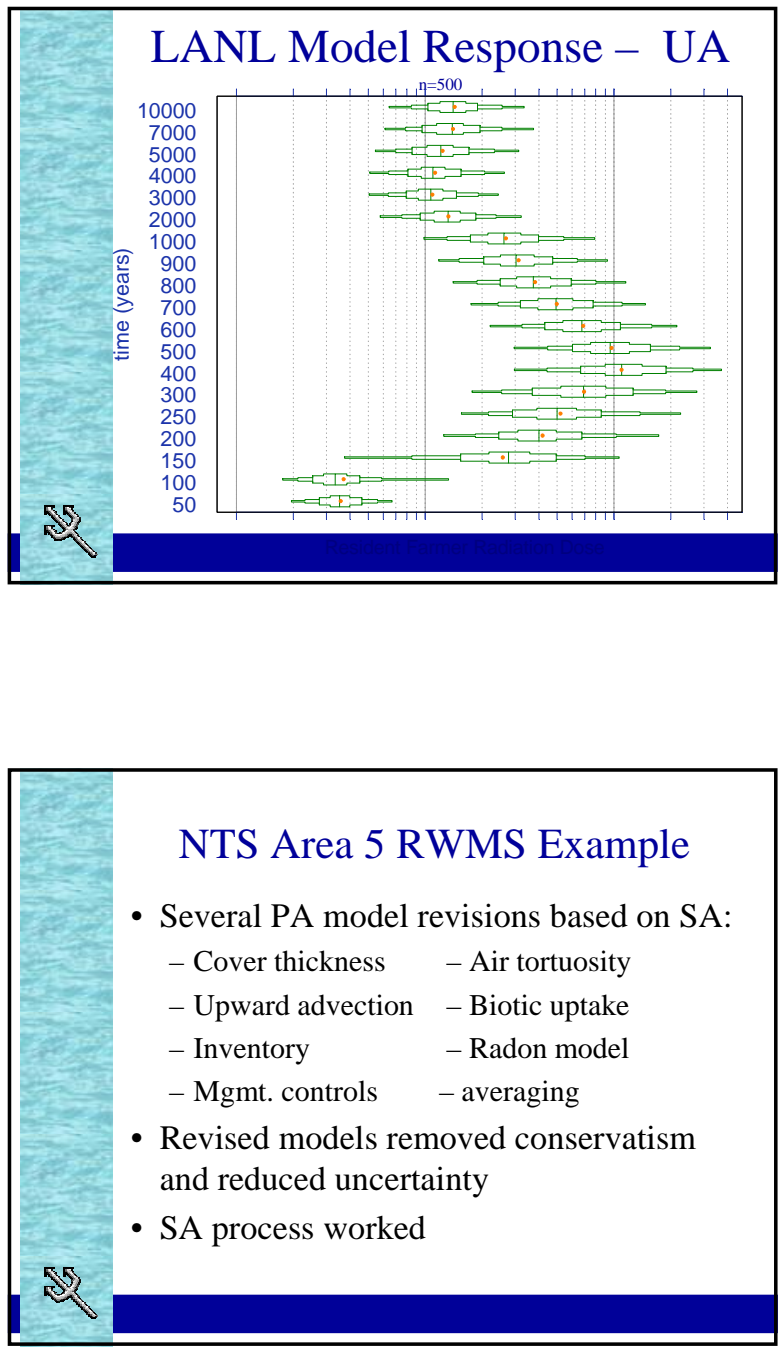

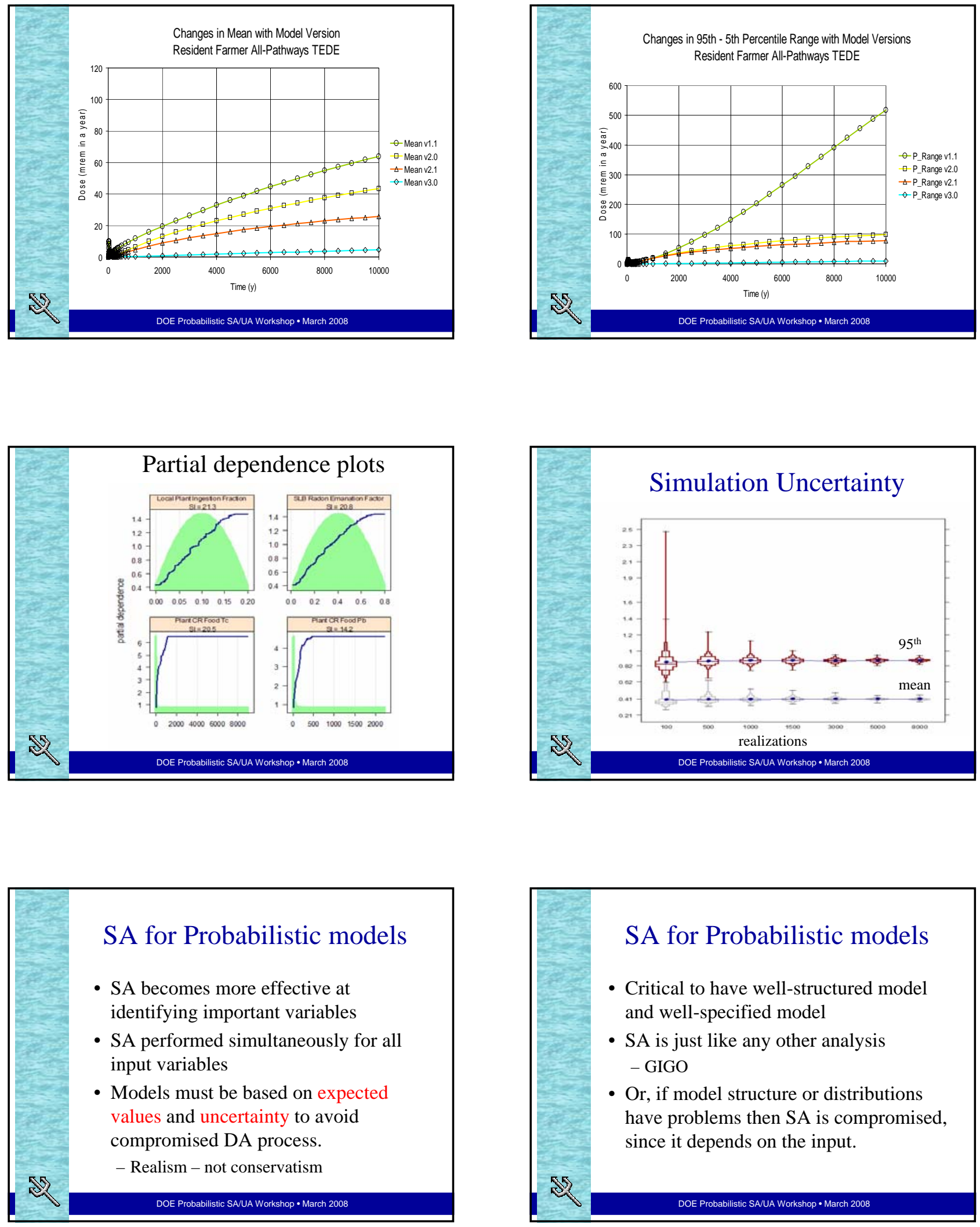


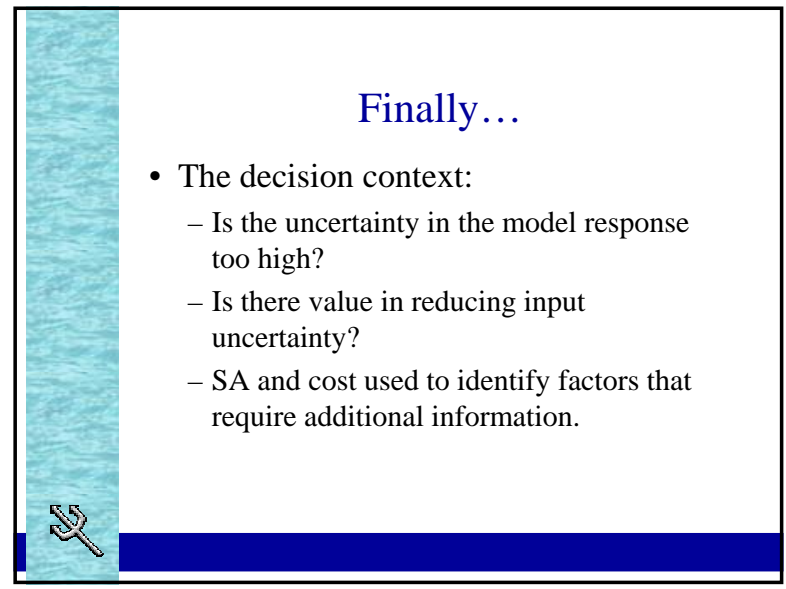

\section{New Guidance?}

- SA (and UA) guidance for interpreting and applying the results of probabilistic PA/CA models

- With respect to the decisions that need to be made

\section{PA Decisions under Uncertainty}

- How much uncertainty can be tolerated?

- Compliance

- Mean, median, 95 ${ }^{\text {th }}$ percentile?

- Future Disposal, Closure

- Trade-off between costs and risk reduction - Use ALARA

- Monitoring, Special Studies

- Value of Information

- Stopping rules

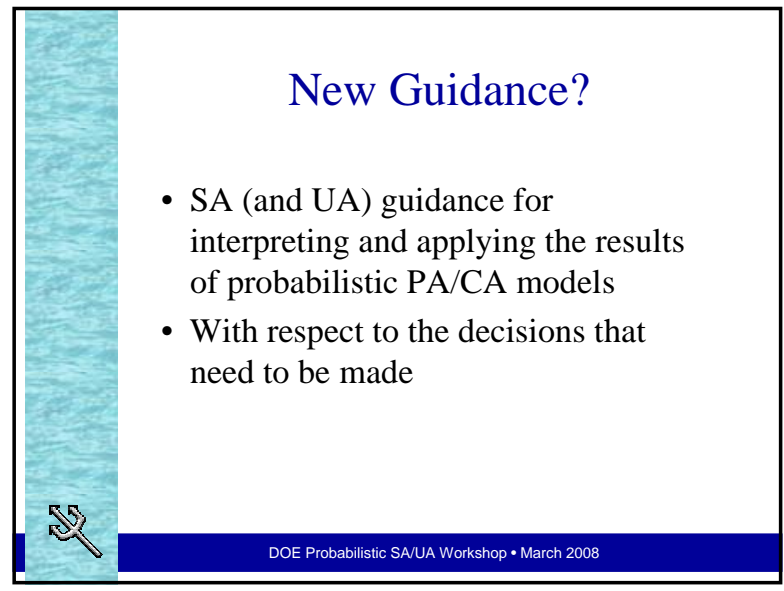


WSRC-STI-2008-00333, REVISION 0

This page intentionally left blank. 